\title{
Proton-Coupled Electron Transfer in the Reduction of Carbonyls by Samarium Diiodide-Water Complexes
}

\author{
Tesia V. Chciuk, William R. Anderson Jr., Robert A. Flowers $\mathrm{II}^{\star^{[a]}}$ \\ Department of Chemistry, Lehigh University, Bethlehem, PA 18015, USA \\ Supporting Information
}

Table of Contents

Experimental

Instrumentation

Materials for Kinetic and Synthetic Reactions

Synthetic Procedures

S3

Kinetic Conditions for $\mathrm{Sml}_{2}-\mathrm{H}_{2} \mathrm{O}$ Reductions and Sample $\boldsymbol{k}_{\mathrm{obs}}$ Determination

Kinetic Conditions and Data for Heptaldehyde Reduction

S5-9

Kinetic Conditions and Data for Cyclohexanone Reduction

S10-14

Kinetic Conditions and Data for 5-Decanolide Reduction

S15-19

UV-Vis Spectra of $\mathrm{Sml}_{2}$-Decanolide

S20

NMR Spectra of Reduced Products 


\section{Experimental Section}

Heptaldehyde and cyclohexanone were distilled and degassed with argon prior to use. 5-Decanolide was purified using a Kügelrohr distillation and then degassed with argon. Substrates were then stored over molecular sieves. Water was degassed with argon overnight. All solutions were prepared inside a drybox containing an argon atmosphere.

UV-Vis spectra were obtained using a Shimadzu UV-1601 spectrophotometer controlled by UV Probe software (version 1.11). Solutions were prepared in a drybox and placed inside an airtight cuvette.

Kinetic experiments were performed using a computer-controlled SX.18 MV stopped-flow spectrophotometer (Applied Photophysics Ltd. Surrey, UK). The prepared solutions were injected separately into the stopped-flow system from airtight BD syringes prepared in an argon glove box. The cell block and the drive syringes of the stopped flow reaction analyzer were flushed a minimum of three times with dry, deoxygenated THF to make the system anaerobic and were subsequently primed by flushing through one set of syringes with solutions to be analyzed. Between each experiment, the cell block was washed with dilute $\mathrm{HNO}_{3}(2 \mathrm{x}), \mathrm{DI} \mathrm{H}_{2} \mathrm{O}(3 \mathrm{x})$, and THF (3x) before additional anhydrous deoxygenated THF washes (3x). The reaction rates were determined by a single exponential fit of the decay of $10 \mathrm{mM} \mathrm{Sml}_{2}$ at $25^{\circ} \mathrm{C}$ and $560 \mathrm{~nm}$ using Pro-Data SX software.

\section{Instrumentation:}

Proton NMR spectra were recorded on a Bruker $500 \mathrm{MHz}$ spectrometer in $\mathrm{CDCl}_{3}$. Carbon NMR were performed at $125 \mathrm{MHz}$ in $\mathrm{CDCl}_{3}$. GC-MS analyses were done with an HP 5890 Series II Gas Chromatograph with an HP Mass Selector Detector. GC analyses were done using a Shimadzu Gas Chromatograph GC-14B with biphenyl standard. Kinetic experiments were performed with a computer-controlled SX.18 MV stopped-flow spectrophotometer (Applied Photophysics Ltd. Surrey, UK). ITC data was obtained using a MicroCal VP-ITC.

\section{Materials for Synthetic and Kinetic Studies:}

Samarium powder was purchased from Alfa Aesar. $\mathrm{Sml}_{2}$ was generated by the standard method of samarium metal combined with iodine in THF and allowed to stir for at least 4 hours. lodometric titrations were performed to verify concentration of $\mathrm{Sml}_{2}$. Tetrahydrofuran was purified by a Solvent Purification system (Innovative Technology Inc.; $\mathrm{MA}$ ). $\mathrm{H}_{2} \mathrm{O}$ and $\mathrm{D}_{2} \mathrm{O}$ were deoxygenated by bubbling through with argon overnight. 


\section{Synthetic Procedure for the Reduction of Heptaldehyde:}

Inside an Ar glove box, $18 \mathrm{~mL}$ of $0.1 \mathrm{M} \mathrm{Sml}_{2}$ was added to a stirring round bottom flask. Water (3.22 $\mathrm{mL}, 0.179 \mathrm{~mol}$ ) was slowly added to produce the characteristic purple color of $\mathrm{Sml}_{2}$-water. To this solution, $100 \mu \mathrm{L}$ of heptaldehyde $(0.715 \mathrm{mmol})$ was added. The reaction was left until the mixture became colorless and a white precipitate. The round bottom flask was removed from the box and quenched with air and $0.1 \mathrm{M} \mathrm{HCl}$. Heptanol was extracted using diethyl ether. The organic layer was then treated with water, saturated aqueous sodium thiosulfate, and then brine. The remaining solution was then dried with magnesium sulfate, filtered and then solvent was removed by rotary evaporation. Heptanol was analyzed by $1 \mathrm{H} \mathrm{NMR}\left(500 \mathrm{MHz}, \mathrm{CDCl}_{3}\right) \delta(\mathrm{ppm}): 3.63(2 \mathrm{H}, \mathrm{d}), 1.55(2 \mathrm{H}, \mathrm{m}), 1.30(8 \mathrm{H}, \mathrm{m})$, $0.88(3 \mathrm{H}, \mathrm{t}) ; 13 \mathrm{C} \mathrm{NMR}\left(125 \mathrm{MHz}, \mathrm{CDCl}_{3}\right) \delta(\mathrm{ppm}): 62.8,32.2,31.8,29.3,25.6,22.7,14.1$.

\section{Synthetic Procedure for the Reduction of Cyclohexanone:}

Inside an Ar glove box, $24 \mathrm{~mL}$ of $0.1 \mathrm{M} \mathrm{Sml}_{2}$ was added to a stirring round bottom flask. Water ( $4.34 \mathrm{~mL}, 0.2412 \mathrm{~mol}$ ) was slowly added to produce the characteristic purple color of $\mathrm{Sml}_{2}$-water. To this solution, $100 \mu \mathrm{L}$ of cyclohexanone $(0.965 \mathrm{mmol})$ was added. The reaction was left until the mixture became colorless and a white precipitate formed. The round bottom flask was removed from the box and quenched with air and $0.1 \mathrm{M} \mathrm{HCl}$. Cyclohexanol was extracted using diethyl ether. The organic layer was then treated with water, saturated aqueous sodium thiosulfate, and then brine. The remaining solution was then dried with magnesium sulfate, filtered, and then solvent was removed by rotary evaporation to provide clean product. Cyclohexanol was analyzed by $1 \mathrm{H} \mathrm{NMR}\left(500 \mathrm{MHz}, \mathrm{CDCl}_{3}\right) \delta$ (ppm): $3.72(1 \mathrm{H}, \mathrm{s}), 3.59(1 \mathrm{H}, \mathrm{m}), 1.88(2 \mathrm{H}, \mathrm{m}), 1.69(2 \mathrm{H}, 1), 1.52(2 \mathrm{H}, \mathrm{m}), 1.24(2 \mathrm{H}, \mathrm{m}), 1.12(2 \mathrm{H}, \mathrm{m}) ; 13 \mathrm{C}$ NMR (125 MHz, $\left.\mathrm{CDCl}_{3}\right) \delta(\mathrm{ppm}):$ 70.3, 67.96, 65.86, 35.54, 25.5, 24.16.

\section{Synthetic Procedure for the Reduction of 5-Decanolide:}

Inside an Ar glove box, $67 \mathrm{~mL}$ ( 6 equiv vs substrate) of $0.1 \mathrm{M} \mathrm{Sml}_{2}$ in THF was placed in a flask with a magnetic stirrer. To this, $12 \mathrm{~mL}$ of $\mathrm{H}_{2} \mathrm{O}$ (100 equiv vs $\mathrm{Sml}_{2}$ ) was added slowly to produce the characteristic purple $\mathrm{Sml}_{2}-\mathrm{H}_{2} \mathrm{O}$ solution. Then, $200 \mu \mathrm{L}$ of 5 -Decanolide $(1.12 \mathrm{mmol})$ dissolved in $2 \mathrm{~mL}$ of THF was injected into the flask. The reaction was left until the mixture became colorless and a white precipitate formed. The round bottom flask was removed from the box and quenched with air and $0.1 \mathrm{M}$ $\mathrm{HCl}$. 1,5-decanediol was extracted using diethyl ether. The organic layer was then washed with water followed by saturated aqueous sodium thiosulfate, and then brine. The remaining solution was then dried with magnesium sulfate, filtered and then solvent was removed by rotary evaporation. 1,5Decanediol was analyzed by $1 \mathrm{H} \mathrm{NMR}\left(500 \mathrm{MHz}^{\mathrm{C}} \mathrm{CDCl}_{3}\right) \delta(\mathrm{ppm}): 3.65(3 \mathrm{H}, \mathrm{m}), 2.31(2 \mathrm{H}$, broad s), 1.5-1.3 $(14 \mathrm{H}, \mathrm{m}), 0.89(3 \mathrm{H}, \mathrm{t}) ; 13 \mathrm{C} \mathrm{NMR}\left(125 \mathrm{MHz}, \mathrm{CDCl}_{3}\right) \delta(\mathrm{ppm}): 72.13,62.78,38.03,36.82,32.60(2 \mathrm{C}), 25.65$, 23.24, 223.5, 14.19 . 


\section{Kinetic Conditions for $\mathrm{Sml}_{2}-\mathrm{H}_{2} \mathrm{O}$ Reductions:}

Kinetic experiments were performed with a computer-controlled SX.18 MV stopped-flow spectrophotometer (Applied Photophysics Ltd. Surrey, UK). The $\mathrm{Sml}_{2}$, substrate, and proton donor solutions were injected independently into the stopped-flow system from airtight BD syringes prepared in a glove box. The cell block and the drive syringes of the stopped flow reaction analyzer were flushed a minimum of three times with dry, degassed THF to make the system anaerobic. The reaction rates were determined from the decay of $\mathrm{Sml}_{2}$ at $25{ }^{\circ} \mathrm{C}$ and $560 \mathrm{~nm}$. Precipitation or phase separation were not observed in any cases (even at high concentrations of water) for any substrates. All concentrations of water provided clean exponential decays over three half lives.

\section{Sample Determination of $\boldsymbol{k}_{\mathrm{obs}}$ under Pseudo-first Order Conditions:}

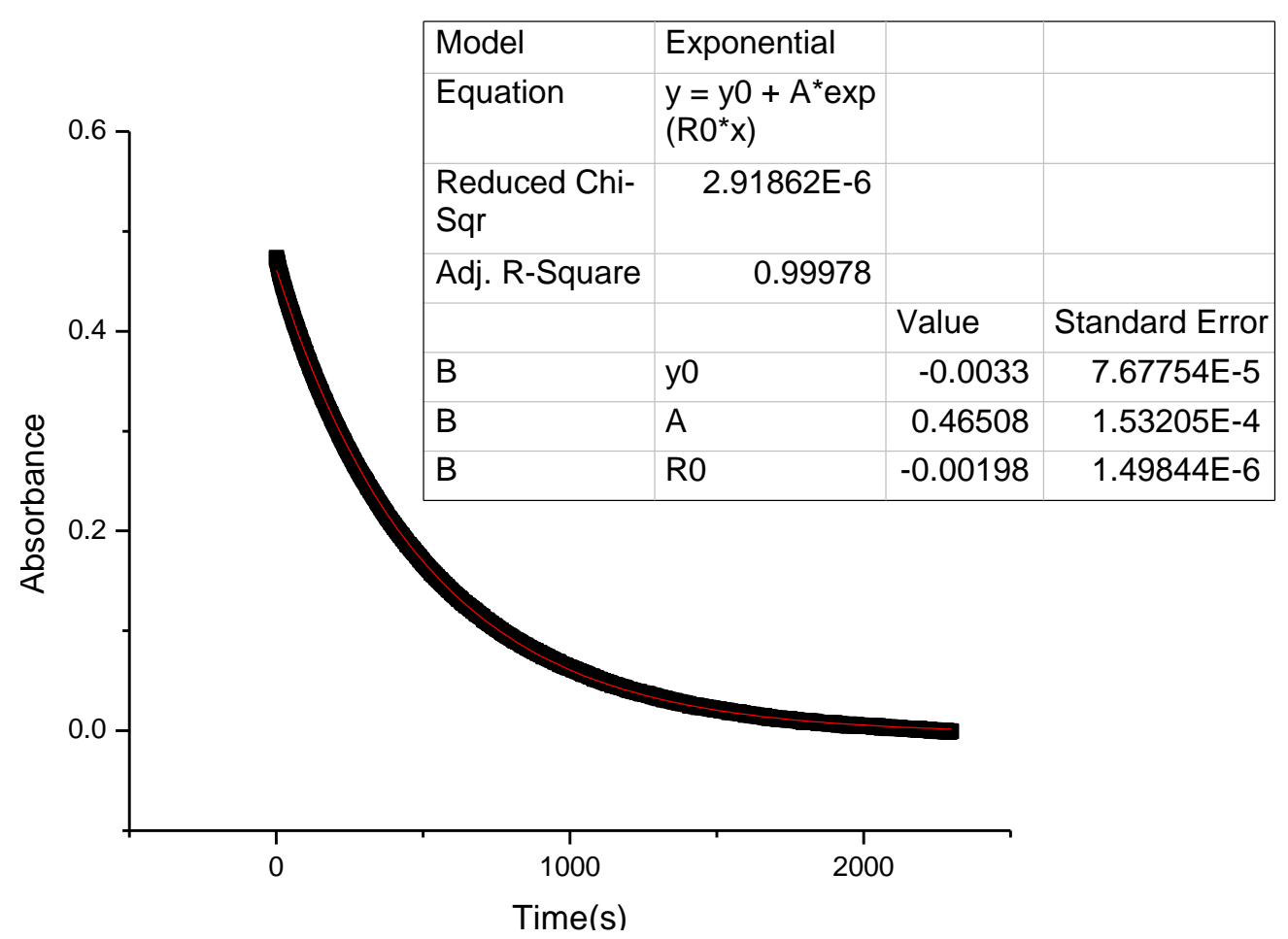

Figure S1. The decay of Sm(II) at $560 \mathrm{~nm}$ where all other reactants are in excess is measured and fit to a single exponential equation where $y=y 0+A^{\left(R 0^{*} x\right)}$ and $k_{o b s}$ is represented by $|R O|$. 


\section{Kinetic Data for Reduction of Heptaldehyde:}

Sample Decay $\mathrm{Sml}_{2}$ in reduction of heptaldehyde:

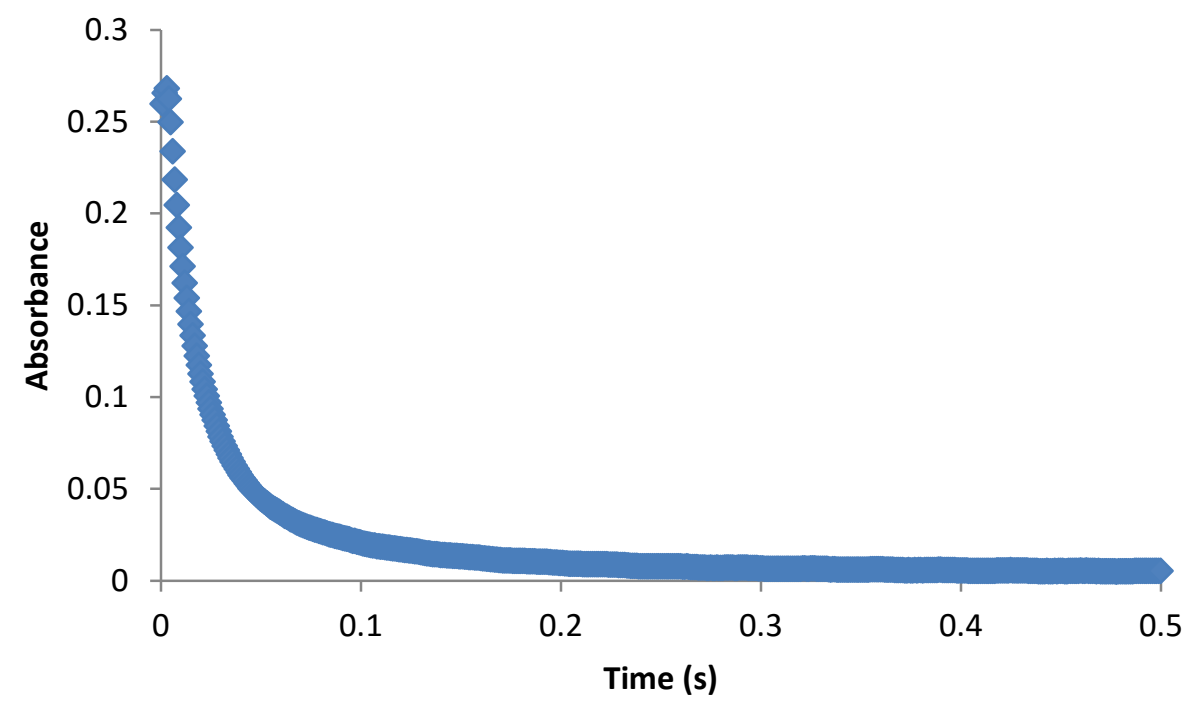

Figure S2. Sample decay of $\mathrm{Sm}(\mathrm{II})$ at $560 \mathrm{~nm}, 25{ }^{\circ} \mathrm{C}$, with $100 \mathrm{mM}$ Heptaldehyde, $1 \mathrm{M} \mathrm{H}_{2} \mathrm{O}$ and $10 \mathrm{mM}$ $\mathrm{Sml}_{2}$. 


\section{Order of Water:}

For the order of water, the concentration of heptaldehyde was kept constant at $100 \mathrm{mM}$ and was combined with $10 \mathrm{mM} \mathrm{Sml}_{2}$. The concentration of water was varied from 50 equivalents to 750 equivalents. The experiment was repeated three times on different dates.

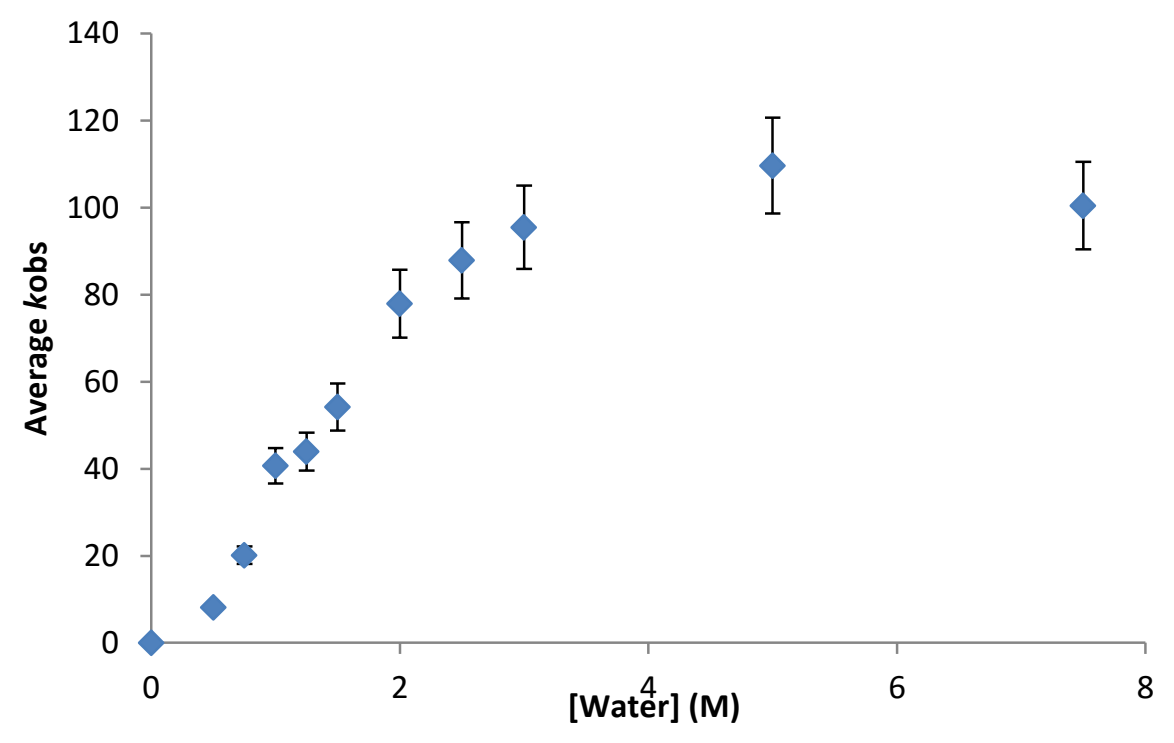

Figure S3. Rates for the reduction of heptaldehyde with increasing concentration of water.

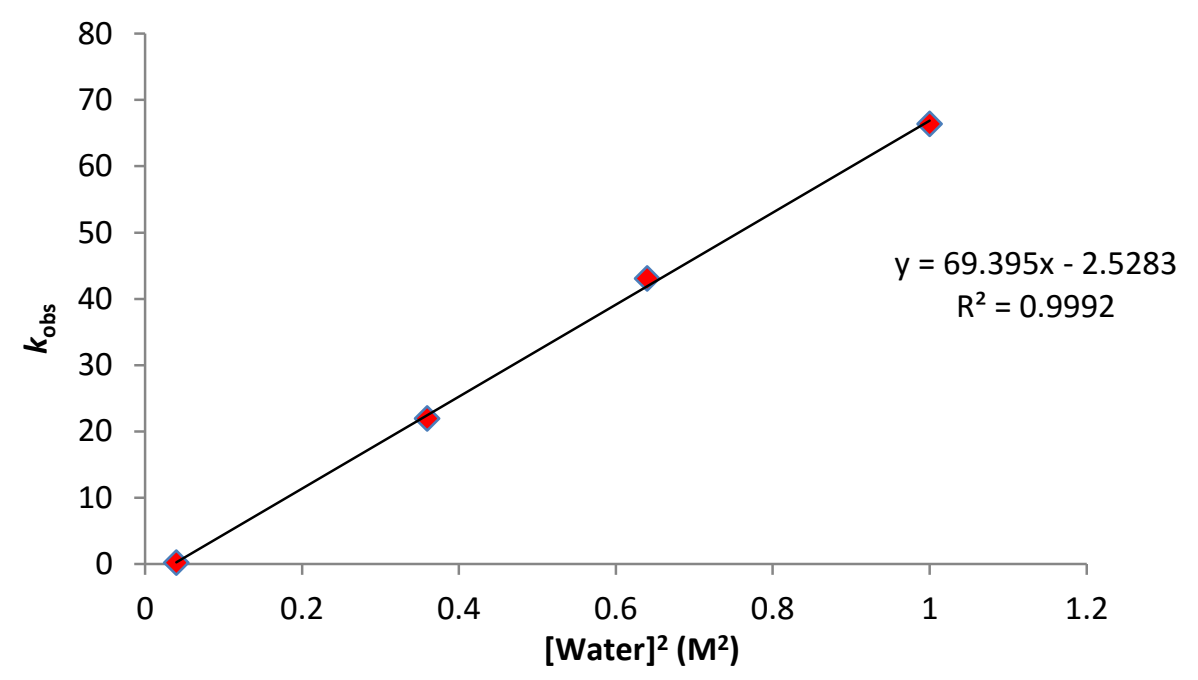

Figure S4. Second-order rate dependence for the reduction of heptaldehyde with increasing concentrations of water at low concentrations.

\section{Order of Heptaldehyde:}


For the order of heptaldehyde, substrate concentration was varied from $80 \mathrm{mM}$ to $160 \mathrm{mM}$. Substrate was combined with $10 \mathrm{mM} \mathrm{Sml}_{2}$ and 100 eq $\mathrm{H}_{2} \mathrm{O}$ in the stopped-flow and the decay of Sm(II) was observed. The experiment was repeated three times on different dates.

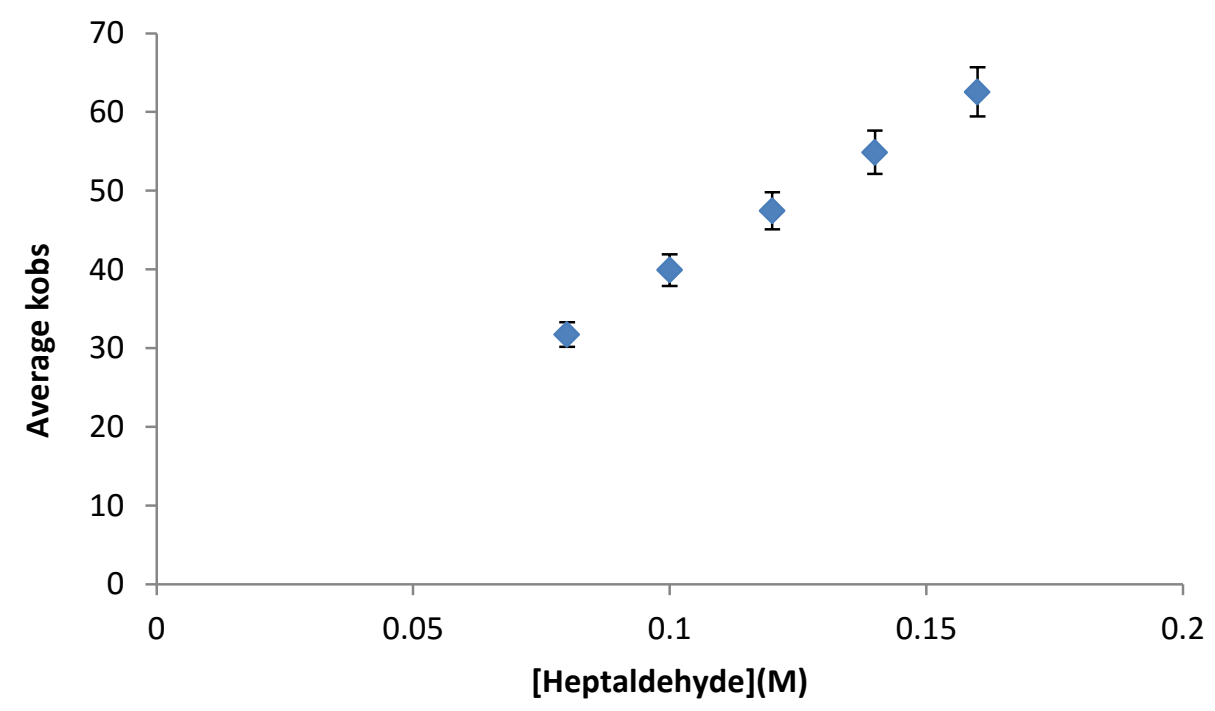

Figure S5. Rates for the reduction of heptaldehyde with increasing concentration of heptaldehyde.

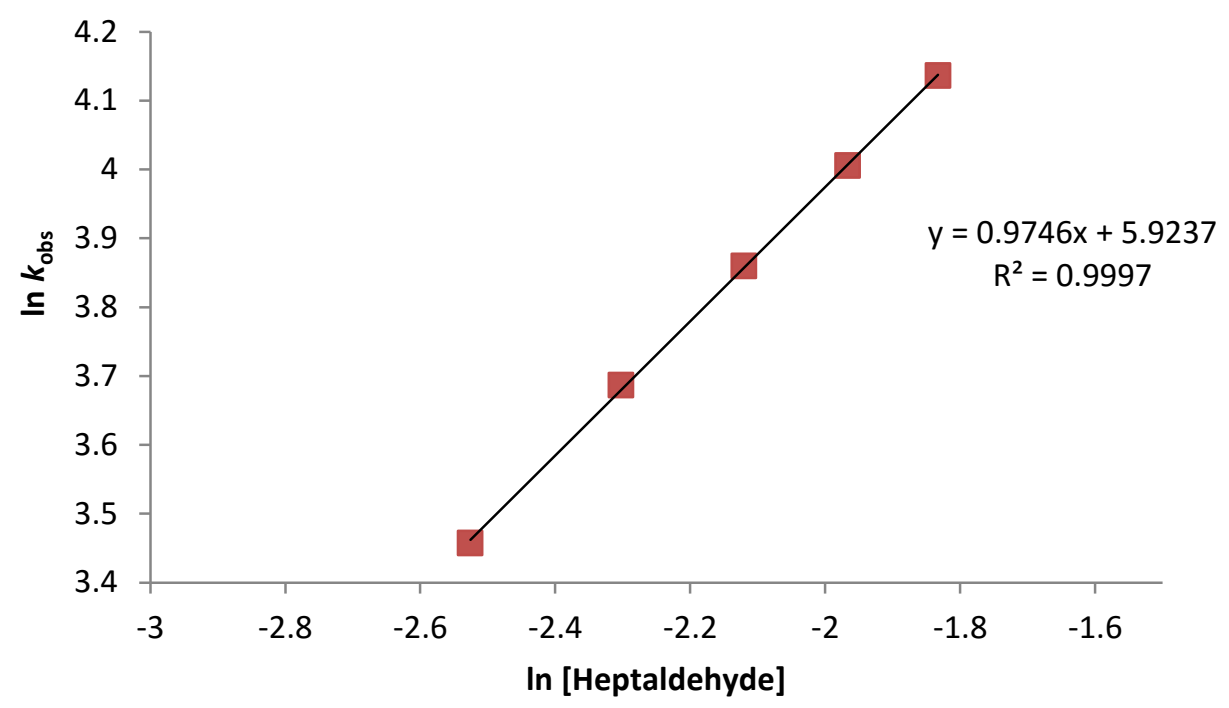

Figure S6. Linear rate dependence for the reduction of heptaldehyde with increasing concentration of heptaldehyde. 


\section{Kinetic Isotope Effect:}

For KIE studies, the concentration of heptaldehyde was kept constant at $100 \mathrm{mM}$ and was combined with $10 \mathrm{mM} \mathrm{Sml}_{2}$. The concentration of $\mathrm{D}_{2} \mathrm{O}$ was varied from 50 equivalents to 750 equivalents (0.5-7.5 M).

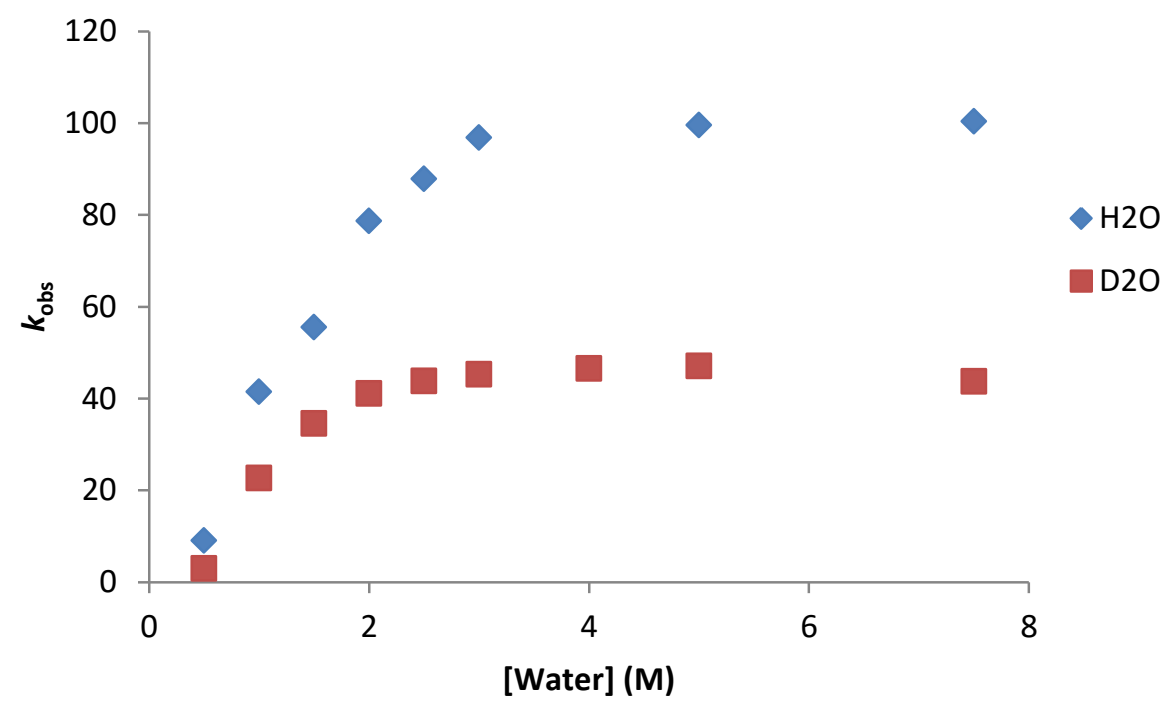

Figure S7. Rates of reduction of heptaldehyde with increasing concentrations of $\mathrm{H}_{2} \mathrm{O}$ and $\mathrm{D}_{2} \mathrm{O}$.

\section{Order of $\mathrm{Sml}_{2}$ using Fractional Times Method:}

Fractional times method was applied to determine the order of $\mathrm{Sml}_{2}$ over more than one day and at different water concentrations with a constant concentration of $100 \mathrm{mM}$ Heptaldehyde and 10

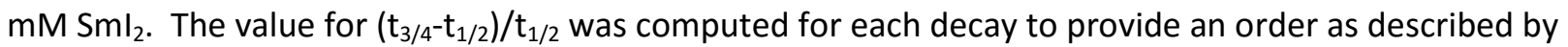
House below.

Table S1. Order of $\mathrm{Sml}_{2}$ in the reduction of heptaldehyde by fractional times method.

\begin{tabular}{|c|c|c|c|c|c|c|c|}
\hline Date & Trial & $A_{0}$ & $A_{1 / 2}$ & $A_{3 / 4}$ & $t_{1 / 2}$ & $t_{3 / 4}$ & $\left(t_{3 / 4}-t_{1 / 2}\right) / t_{1 / 2}$ \\
\hline 01-14-16 & $100 \mathrm{~A}$ & 0.28322 & 0.14161 & 0.071 & 0.022 & 0.043 & 0.95 \\
\hline 01-19-16 & $100 \mathrm{~A}$ & 0.291892 & 0.145946 & 0.072973 & 0.016 & 0.038 & 1.38 \\
\hline 01-19-16 & $125 \mathrm{~A}$ & 0.250474 & 0.125237 & 0.0626185 & 0.014 & 0.032 & 1.29 \\
\hline & & & & & & Average: & 1.2 \\
\hline & & & & & & Order: & 1 \\
\hline
\end{tabular}

House, J. E. Principles of Chemical Kinetics; Academic Press: St. Louis, MO, 2007; pp 79-83. 
Activation Parameters for Heptaldehyde:

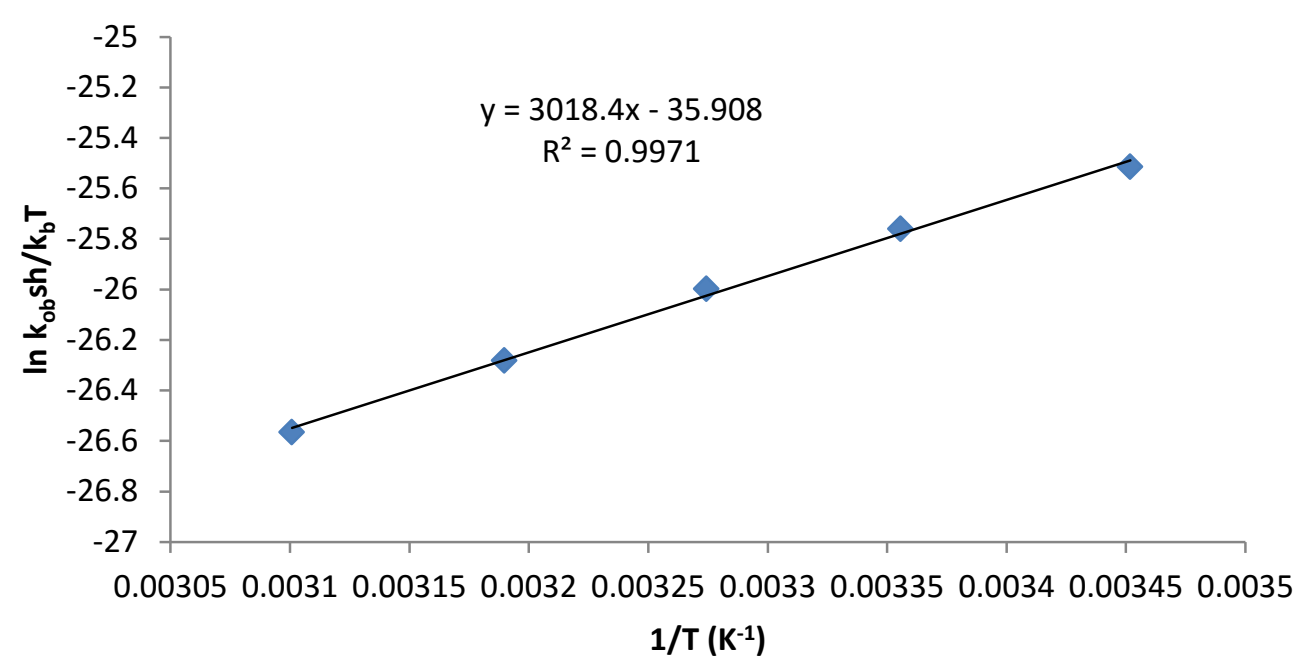

Figure S8. Sample eyring plot for the reduction of $100 \mathrm{mM}$ heptaldehyde with $1 \mathrm{M} \mathrm{H}_{2} \mathrm{O}$ and $10 \mathrm{mM} \mathrm{Sml}_{2}$ from $20-50{ }^{\circ} \mathrm{C}$. This experiment was performed three times and the reported activation parameters are an average of the values obtained. 


\section{Kinetic Conditions for Reduction of Cyclohexanone:}

Kinetic experiments were performed with a computer-controlled SX.18 MV stopped-flow spectrophotometer (Applied Photophysics Ltd. Surrey, UK). The cyclohexanone and $\mathrm{Sml}_{2}$-water solutions were injected separately into the stopped-flow system from airtight BD syringes prepared in a glove box. The cell block and the drive syringes of the stopped flow reaction analyzer were flushed a minimum of three times with dry, degassed THF to make the system anaerobic. The reaction rates were determined from the decay of $\mathrm{Sml}_{2}$ at $560 \mathrm{~nm}$.

Reactions were monitored at $25 \pm 0.5^{\circ} \mathrm{C}$.

Sample Decay $\mathrm{Sml}_{2}-\mathrm{H}_{2} \mathrm{O}-$ Cyclohexanone:

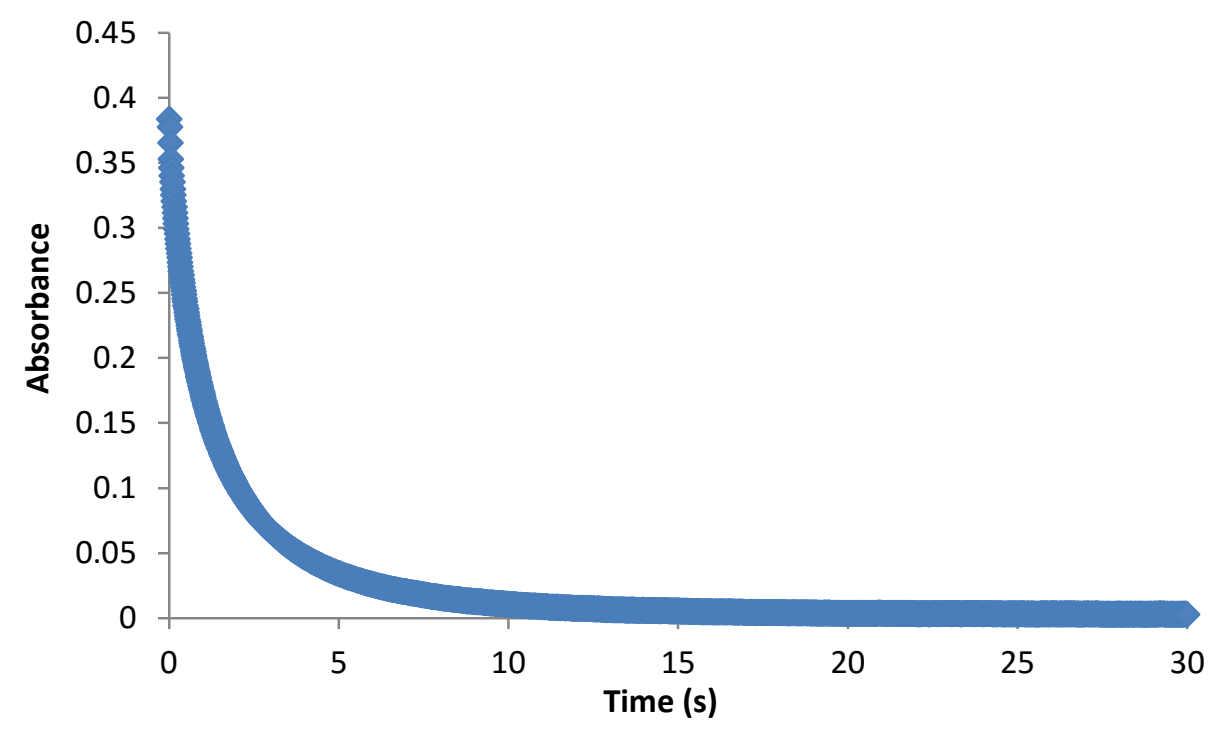

Figure S9. Sample decay for $1 \mathrm{M} \mathrm{H}_{2} \mathrm{O}, 100 \mathrm{mM}$ Cyclohexanone, $10 \mathrm{mM} \mathrm{Sml}_{2}$, measured at $25{ }^{\circ} \mathrm{C}, 560 \mathrm{~nm}$. 


\section{Order of Water:}

For the order of water, the concentration of cyclohexanone was kept constant at $100 \mathrm{mM}$ and was combined with $10 \mathrm{mM} \mathrm{Sml}_{2}$. Each experiment was repeated three times.

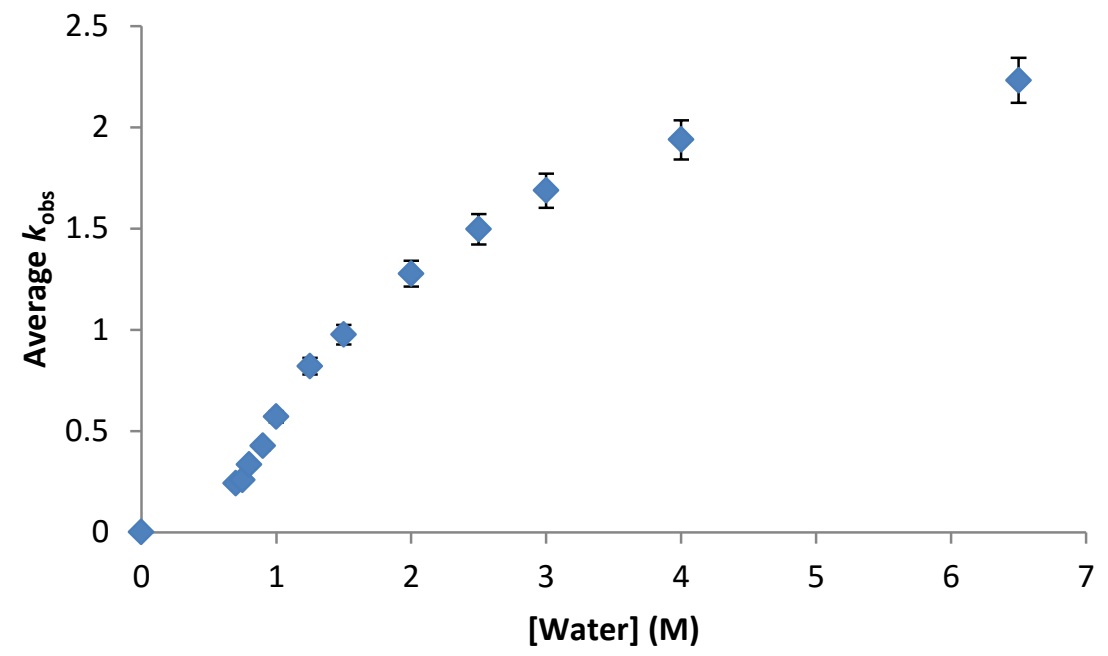

Figure S10. Rates of reduction of cyclohexanone with increasing concentration of water.

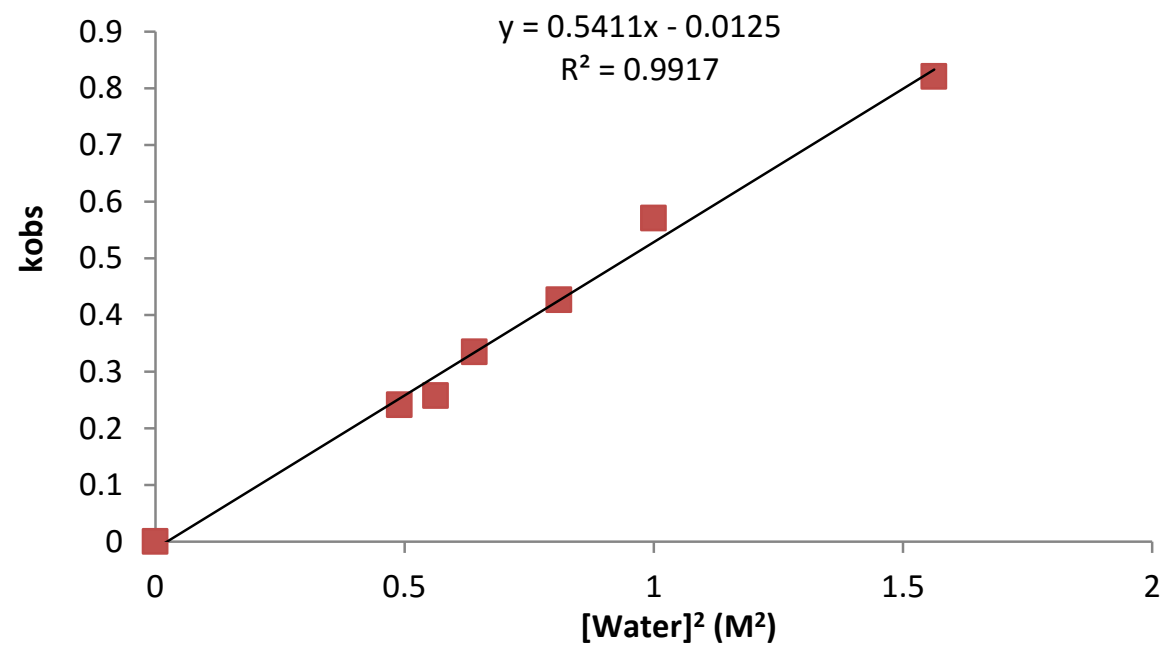

Figure S11. Second-order rate dependence on water in the reduction of cyclohexanone at low concentrations. 


\section{Order of Cyclohexanone:}

For the order of cyclohexanone, substrate concentration was varied from $100 \mathrm{mM}$ to $500 \mathrm{mM}$. Substrate was combined with $10 \mathrm{mM} \mathrm{Sml}_{2}$ and $100 \mathrm{eq}_{2} \mathrm{O}$ in the stopped-flow and the decay of Sm(II) was observed at $560 \mathrm{~nm}$ and $25 \pm 0.5^{\circ} \mathrm{C}$. The experiment was repeated three times.

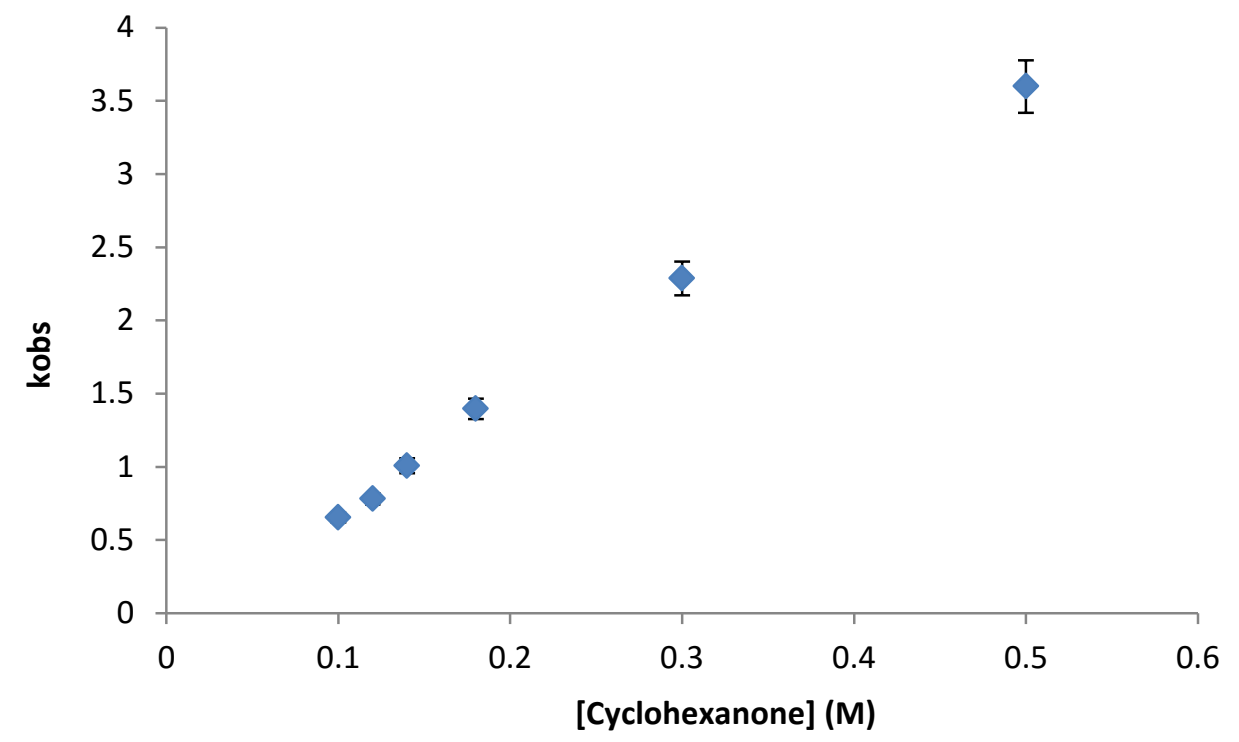

Figure S12. Linear rate dependence on cyclohexanone with increasing concentrations of cyclohexanone

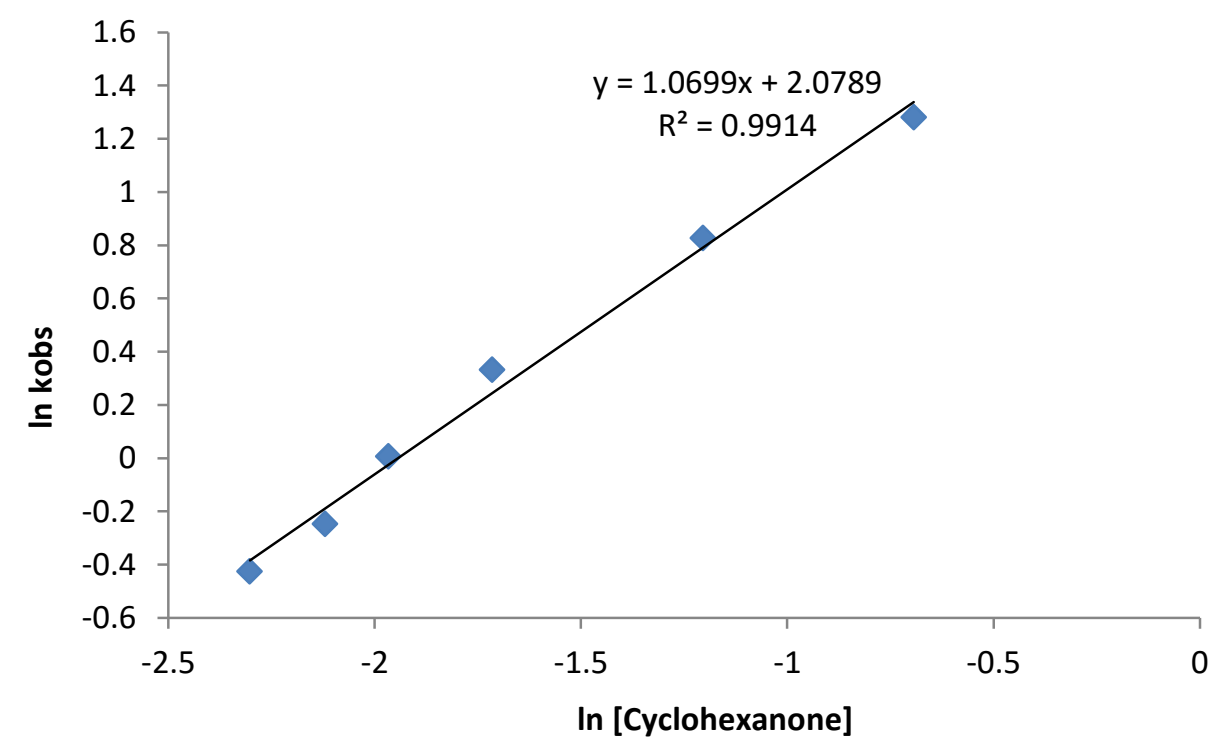

Figure S13. Linear rate dependence on cyclohexanone with increasing concentrations of cyclohexanone. 


\section{Order of $\mathrm{Sml}_{2}$ using Fractional Times Method:}

Fractional times method was applied to determine the order of $\mathrm{Sml}_{2}$ over more than one day and at different water concentrations with a constant concentration of $100 \mathrm{mM}$ cyclohexanone and 10 $\mathrm{mM} \mathrm{Sml}$. The value for $\left(\mathrm{t}_{3 / 4}-\mathrm{t}_{1 / 2}\right) / \mathrm{t}_{1 / 2}$ was computed for each decay to provide an order as described by House below.

Table S2. Order of $\mathrm{Sml}_{2}$ in the reduction of cyclohexanone by fractional times method.

\begin{tabular}{|c|c|c|c|c|c|c|c|}
\hline Date & File & $A_{0}$ & $A_{1 / 2}$ & $A_{3 / 4}$ & $t_{1 / 2}$ & $t_{3 / 4}$ & $\left(t_{3 / 4}-t_{1 / 2}\right) / t_{1 / 2}$ \\
\hline $10-26-15$ & $100 \mathrm{~A}$ & 0.327913 & 0.163957 & 0.081978 & 1.75 & 3.75 & 1.14 \\
\hline $11-06-15$ & $150 \mathrm{~A}$ & 0.321427 & 0.160714 & 0.0804 & 0.7 & 1.4 & 1 \\
\hline \multirow[t]{3}{*}{$11-06-15$} & $250 \mathrm{~A}$ & 0.288823 & 0.144412 & 0.0722056 & 0.5 & 1 & 1 \\
\hline & & & & & & Average: & 1.05 \\
\hline & & & & & & Order: & 1 \\
\hline
\end{tabular}

House, J. E. Principles of Chemical Kinetics; Academic Press: St. Louis, MO, 2007; pp 79-83.

Kinetic Isotope Effect on Reduction of Cyclohexanone:

The kinetic isotope study was performed using equimolar quantities of degassed $\mathrm{H}_{2} \mathrm{O}$ and $\mathrm{D}_{2} \mathrm{O}$ where the water concentration was varied from $0.75-6.5 \mathrm{M}$ and the concentration of cyclohexanone was maintained at $100 \mathrm{mM}$ and $\mathrm{Sml}_{2}$ was maintained at $10 \mathrm{mM}$.

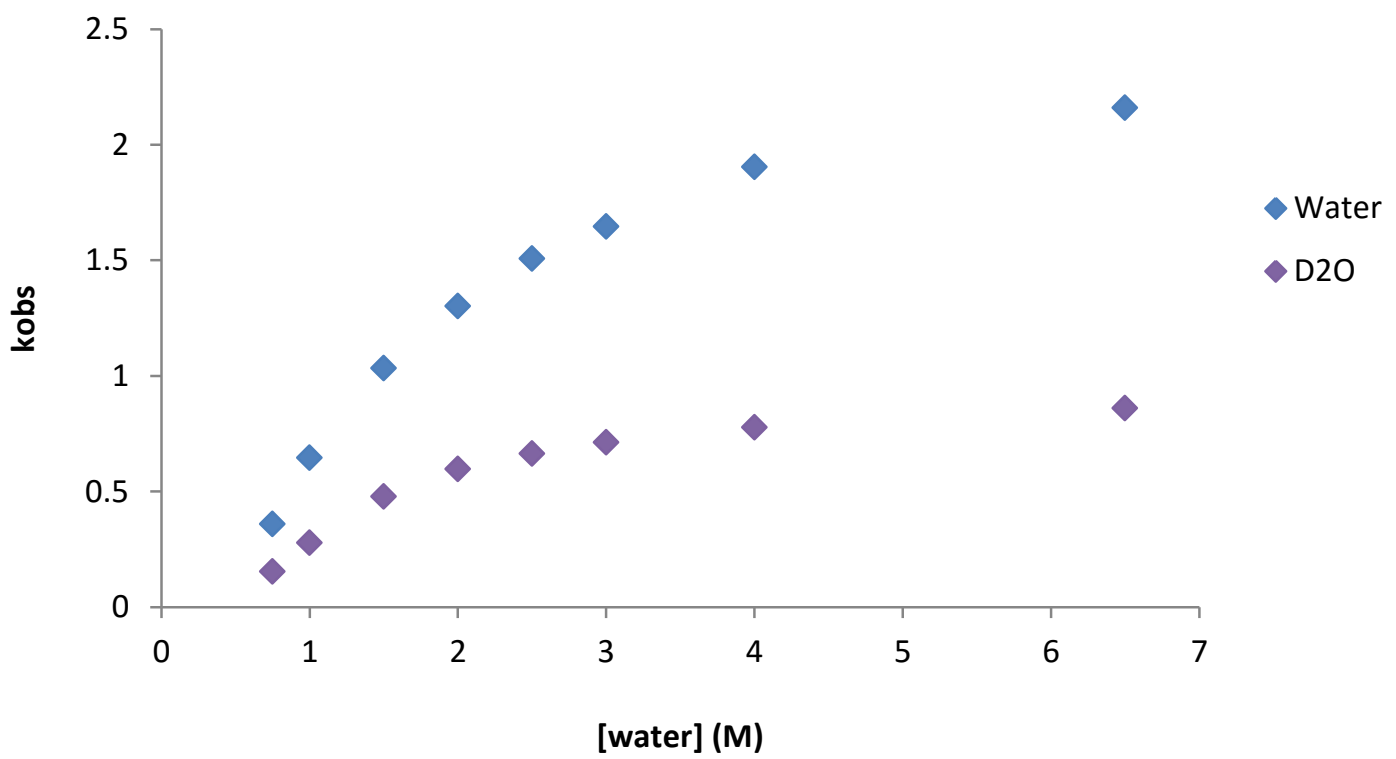

Figure S14. Rates of reduction of cyclohexanone with increasing concentrations of $\mathrm{H}_{2} \mathrm{O}$ and $\mathrm{D}_{2} \mathrm{O}$. 
Activation Parameters for Cyclohexanone:

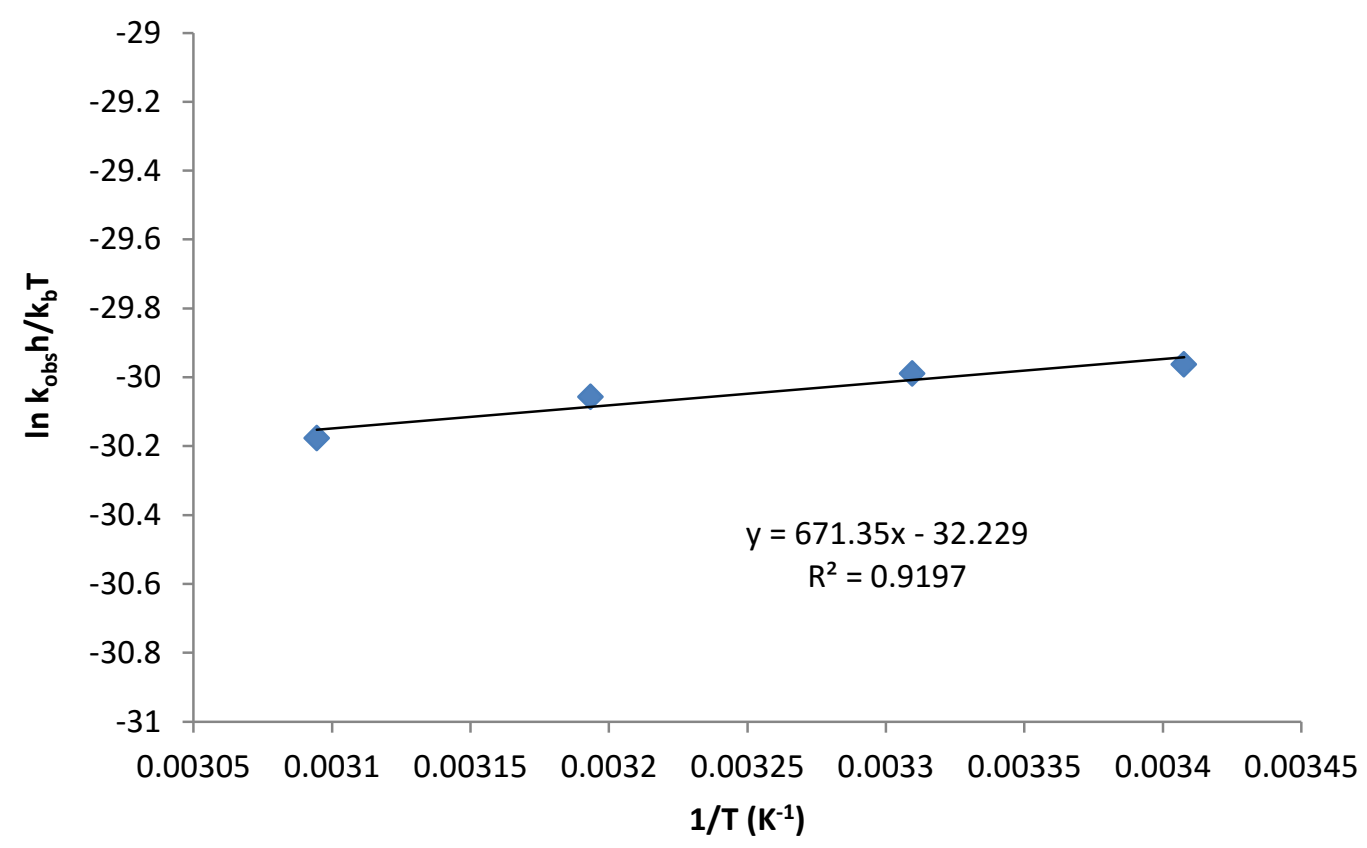

Figure S15. Sample eyring plot for the reduction of $100 \mathrm{mM}$ cyclohexanone with $1 \mathrm{M} \mathrm{H}_{2} \mathrm{O}$ and $10 \mathrm{mM}$ $\mathrm{Sml}_{2}$ from $20-50{ }^{\circ} \mathrm{C}$. This experiment was performed three times and the reported activation parameters are an average of the values obtained. 


\section{Kinetic Conditions for Reduction of 5-Decanolide:}

Kinetic experiments were performed with a computer-controlled SX.18 MV stopped-flow spectrophotometer (Applied Photophysics Ltd. Surrey, UK). The 5-decanolide and $\mathrm{Sml}_{2}$-water solutions were injected separately into the stopped-flow system from airtight BD syringes prepared in a glove box. The cell block and the drive syringes of the stopped flow reaction analyzer were flushed a minimum of three times with dry, degassed THF to make the system anaerobic. The reaction rates were determined from the decay of $\mathrm{Sml}_{2}$ at $560 \mathrm{~nm}$.

Reactions were monitored at $25 \pm 0.5^{\circ} \mathrm{C}$.

Sample Decay $\mathrm{Sml}_{2}-\mathrm{H}_{2} \mathrm{O}-$ Decanolide:

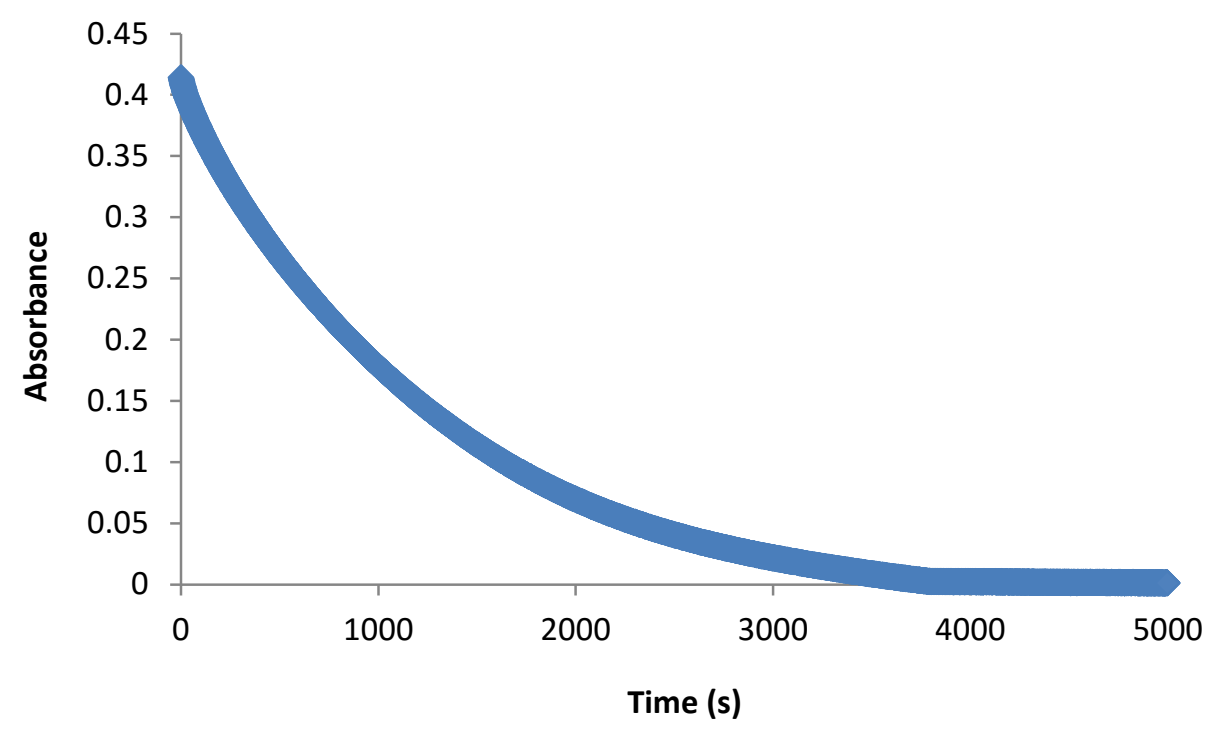

Figure S16. Sample decay for $1 \mathrm{M} \mathrm{H}_{2} \mathrm{O}, 500 \mathrm{mM}$ 5-decanolide, $10 \mathrm{mM} \mathrm{Sml}_{2}$, measured at $25 \mathrm{C}, 560 \mathrm{~nm}$. 


\section{Order of Water:}

For the order of water, the concentration of 5-decanolide was kept constant at $500 \mathrm{mM}$ and was combined with $10 \mathrm{mM} \mathrm{Sml}_{2}$. Each experiment was repeated three times.

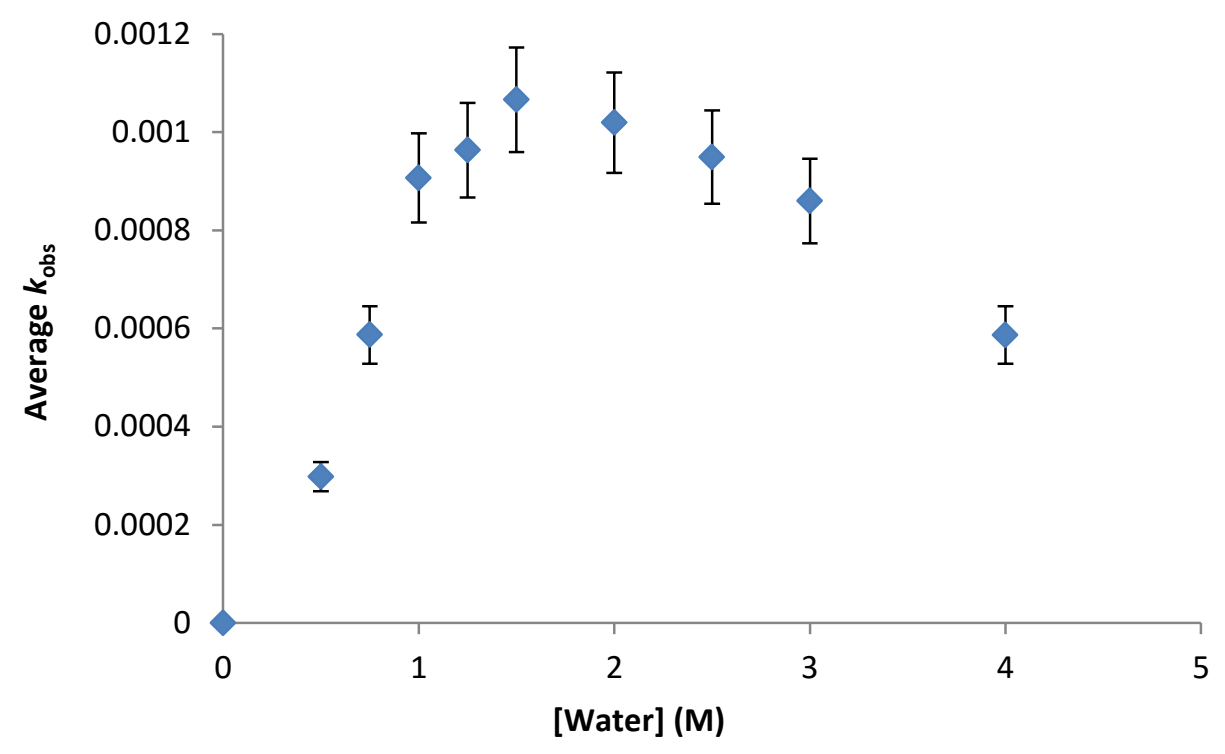

Figure S17. Rates of reduction of 5-decanolide with increasing concentration of water.

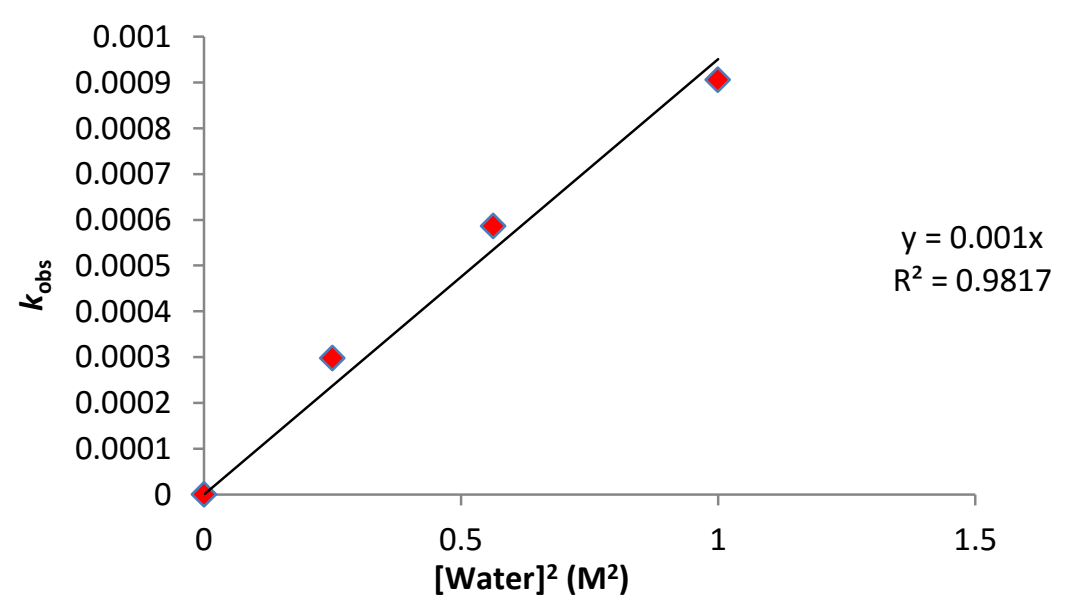

Figure S18. Second-order rate dependence on water in the reduction of 5-decanolide at low concentrations. 


\section{Order of 5-Decanolide:}

For the order of 5-decanolide, substrate concentration was varied from $100 \mathrm{mM}$ to $300 \mathrm{mM}$. Substrate was combined with $10 \mathrm{mM} \mathrm{Sml}_{2}$ and 100 eq $\mathrm{H}_{2} \mathrm{O}$ in the stopped-flow and the decay of Sm(II) was observed at $560 \mathrm{~nm}$ and $25 \pm 0.5^{\circ} \mathrm{C}$. The experiment was repeated three times.

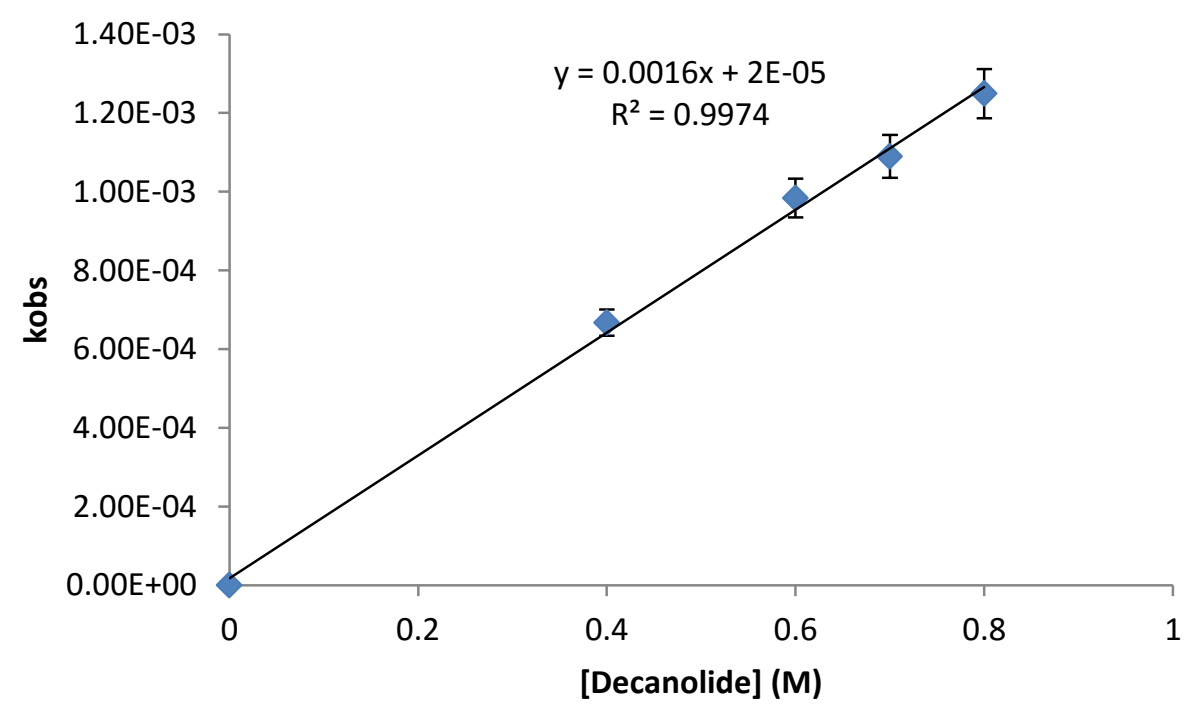

Figure S19. Linear rate dependence on 5-decanolide with increasing concentrations of 5-decanolide.

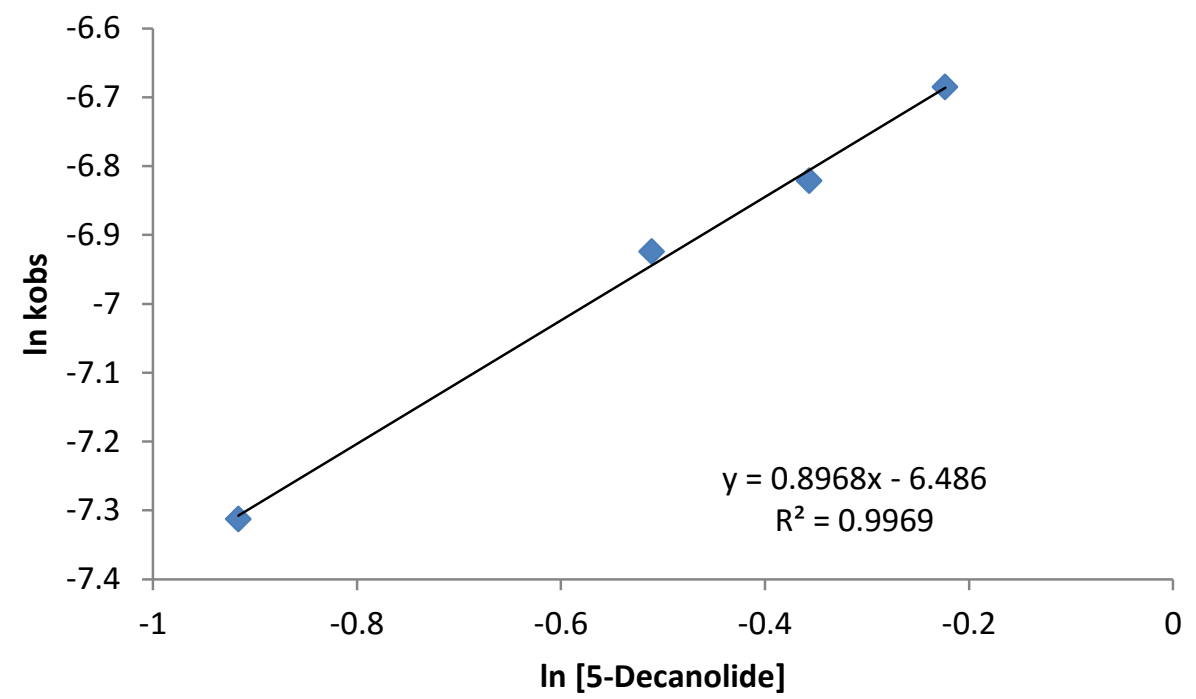

Figure S20. Linear rate dependence on 5-decanolide with increasing concentrations of 5-decanolide. 


\section{Order of $\mathrm{Sml}_{2}$ using Fractional Times Method:}

Fractional times method was applied to determine the order of $\mathrm{Sml}_{2}$ over more than one day and at different water concentrations with a constant concentration of $500 \mathrm{mM} \mathrm{5-decanolide} \mathrm{and} 10$

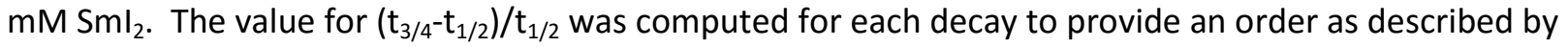
House below.

Table S3. Order of $\mathrm{Sml}_{2}$ in the reduction of 5-decanolide by fractional times method.

\begin{tabular}{|c|c|c|c|c|c|c|c|}
\hline Date & File & $A_{0}$ & $A_{1 / 2}$ & $A_{3 / 4}$ & $t_{1 / 2}$ & $t_{3 / 4}$ & $\left(t_{3 / 4}-t_{1 / 2}\right) / t_{1 / 2}$ \\
\hline 09-08-15 & $100 \mathrm{~A}$ & 0.444386 & 0.222193 & 0.1110965 & 665 & 1343 & 1.02 \\
\hline 09-09-15 & $150 \mathrm{~A}$ & 0.410207 & 0.2051035 & 0.1025518 & 492 & 1006 & 1.05 \\
\hline \multirow[t]{3}{*}{ 09-15-15 } & $200 \mathrm{~A}$ & 0.335609 & 0.1678045 & 0.08390225 & 528 & 1063 & 1.01 \\
\hline & & & & & & Average: & 1.03 \\
\hline & & & & & & Order: & 1 \\
\hline
\end{tabular}

House, J. E. Principles of Chemical Kinetics; Academic Press: St. Louis, MO, 2007; pp 79-83.

Kinetic Isotope Effect on Reduction of 5-Decanolide:

The kinetic isotope study was performed using equimolar quantities of degassed $\mathrm{H}_{2} \mathrm{O}$ and $\mathrm{D}_{2} \mathrm{O}$ where the water concentration was varied from $0.75-3 \mathrm{M}$ and the concentration of 5-decanolide was maintained at $500 \mathrm{mM}$ and $\mathrm{Sml}_{2}$ was maintained at $10 \mathrm{mM}$.

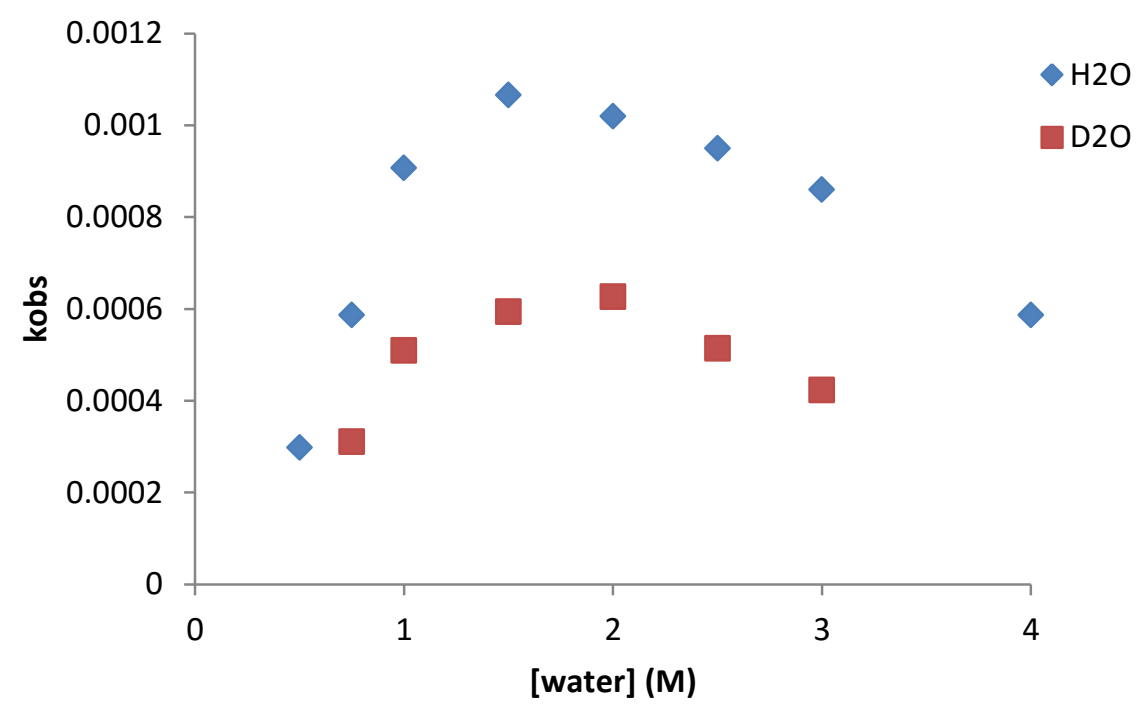

Figure S21. Rates of reduction of 5-decanolide with increasing concentrations of $\mathrm{H}_{2} \mathrm{O}$ and $\mathrm{D}_{2} \mathrm{O}$. 
Activation Parameters for 5-Decanolide:

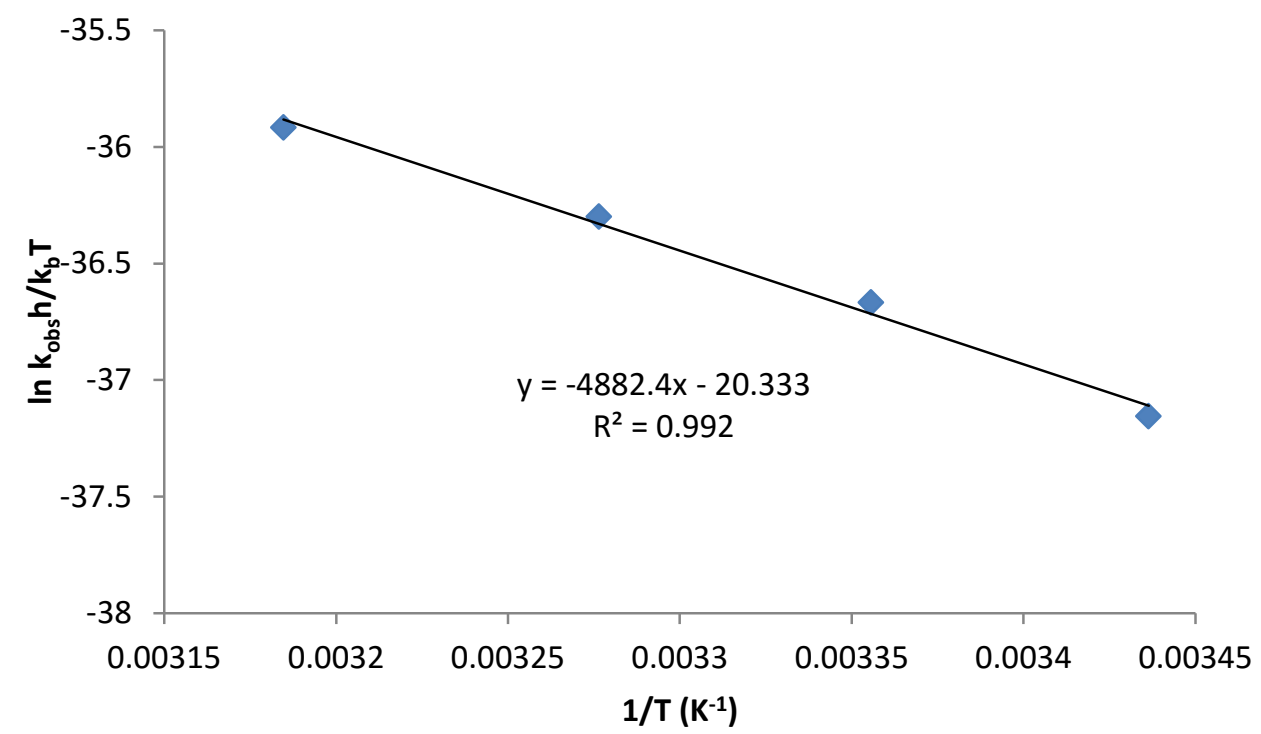

Figure S22. Sample eyring plot for the reduction of $500 \mathrm{mM}$ 5-decanolide with $1 \mathrm{M} \mathrm{H}_{2} \mathrm{O}$ and $10 \mathrm{mM} \mathrm{Sml}_{2}$ from $20-50{ }^{\circ} \mathrm{C}$. This experiment was performed three times and the reported activation parameters are an average of the values obtained. 
UV-Vis Spectra of $\mathrm{Sml}_{2}$-5-decanolide:

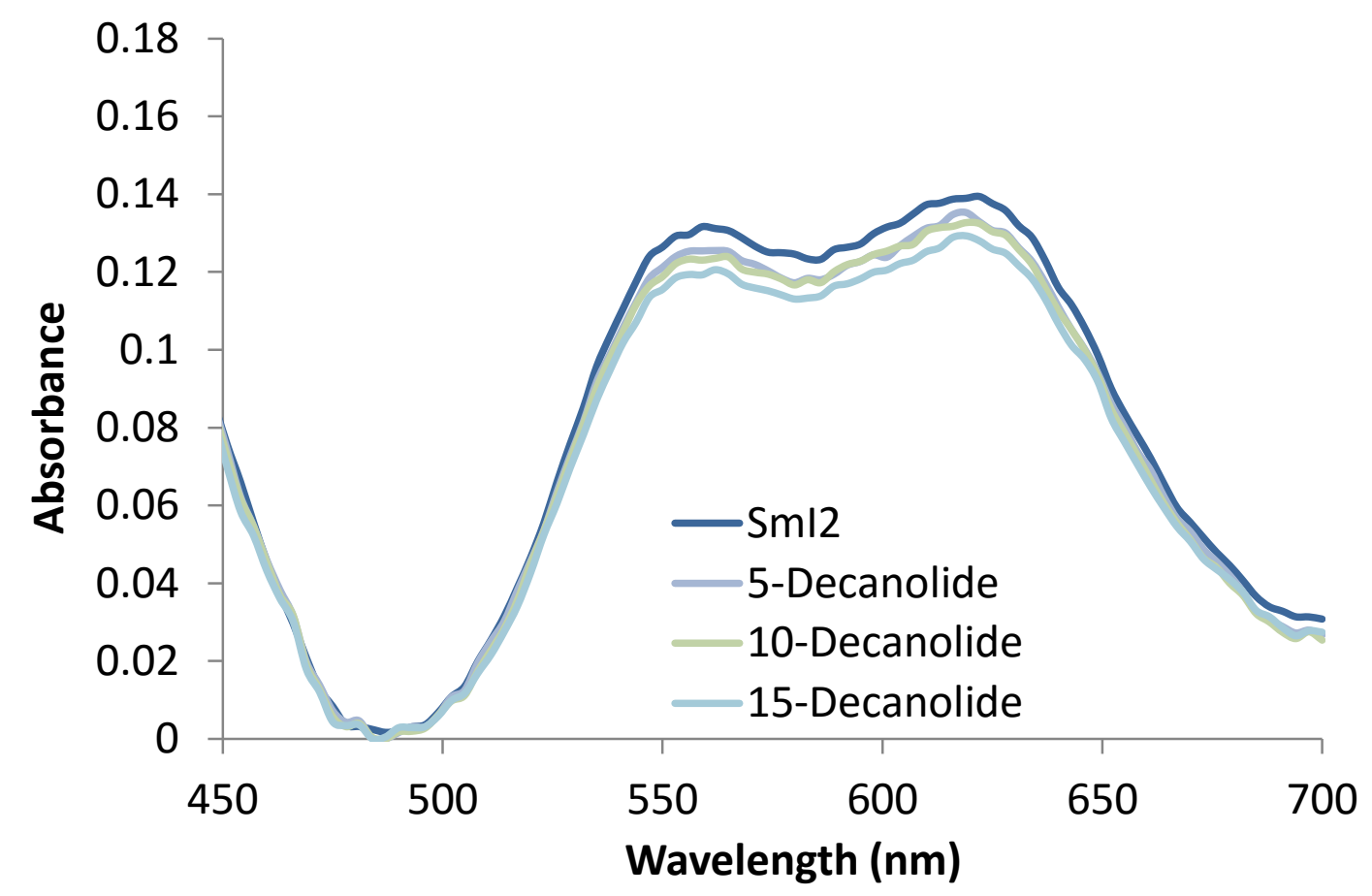

Figure S23: UV-Vis spectra of $\mathrm{Sml}_{2}(2.5 \mathrm{mM})$ in presence of increasing amount of 5-decanolide $(5,10,15$ equiv) in THF. 
NMR Spectra of Reduced Products:

1-Heptanol:

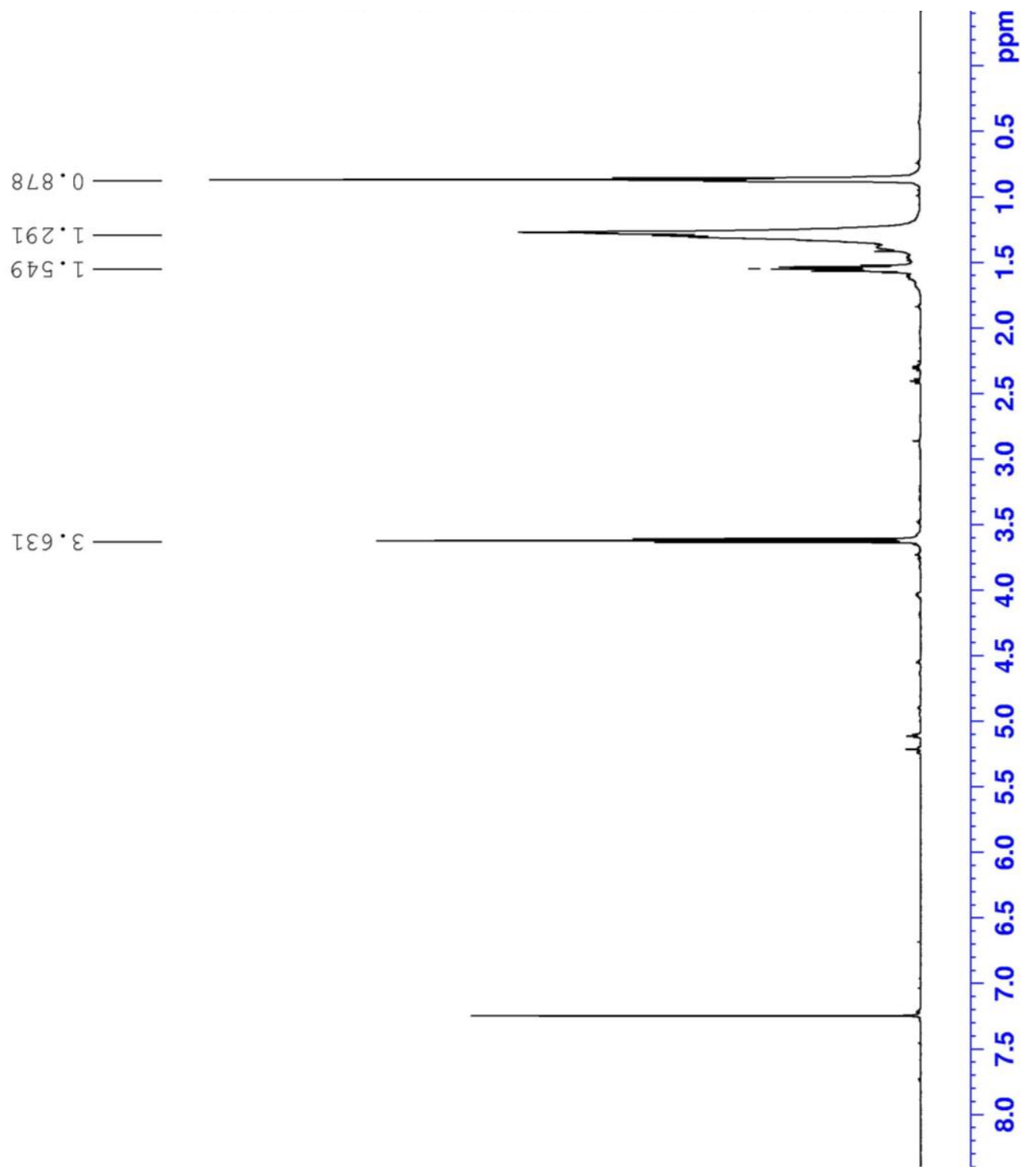




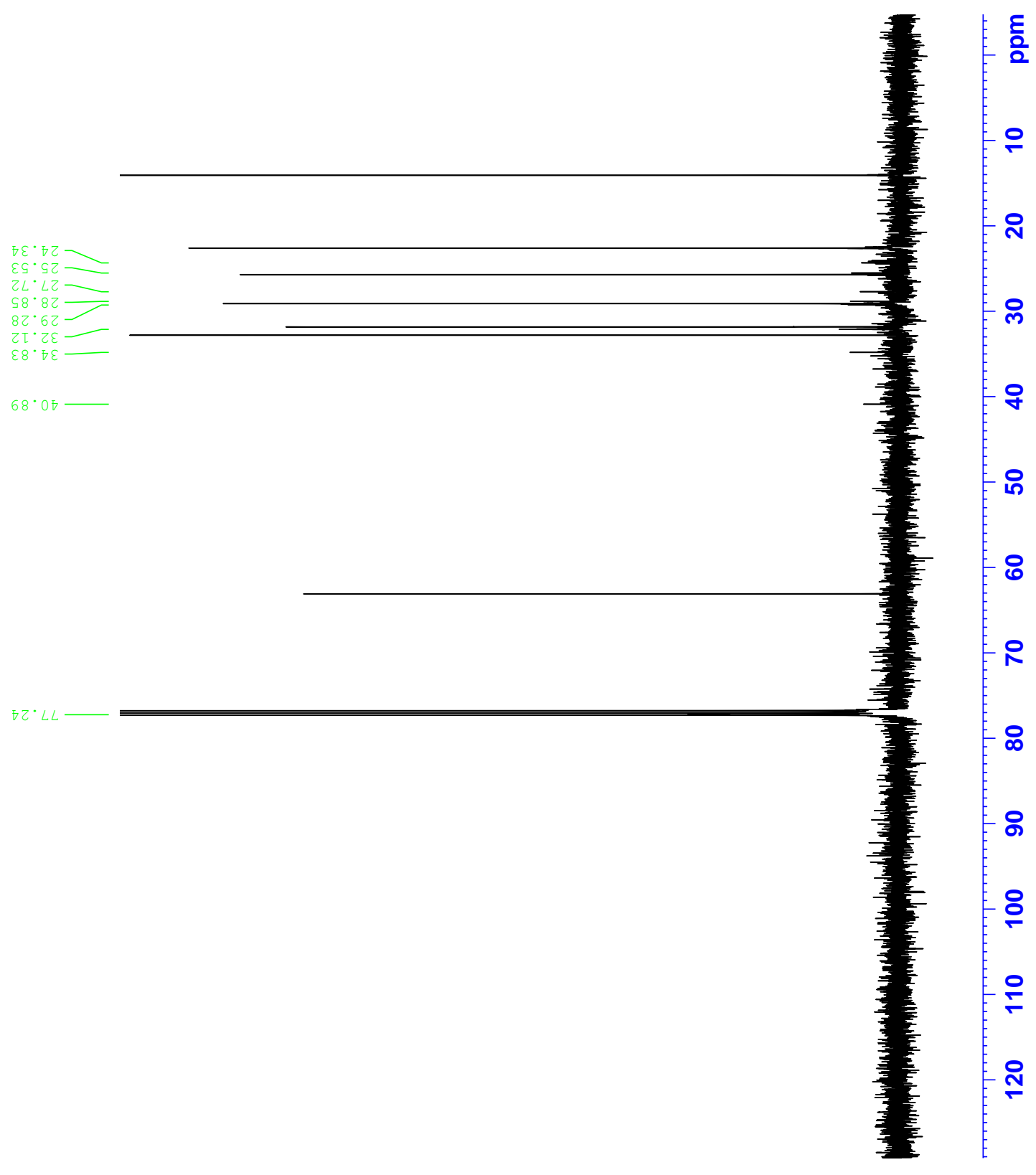


Cyclohexanol:
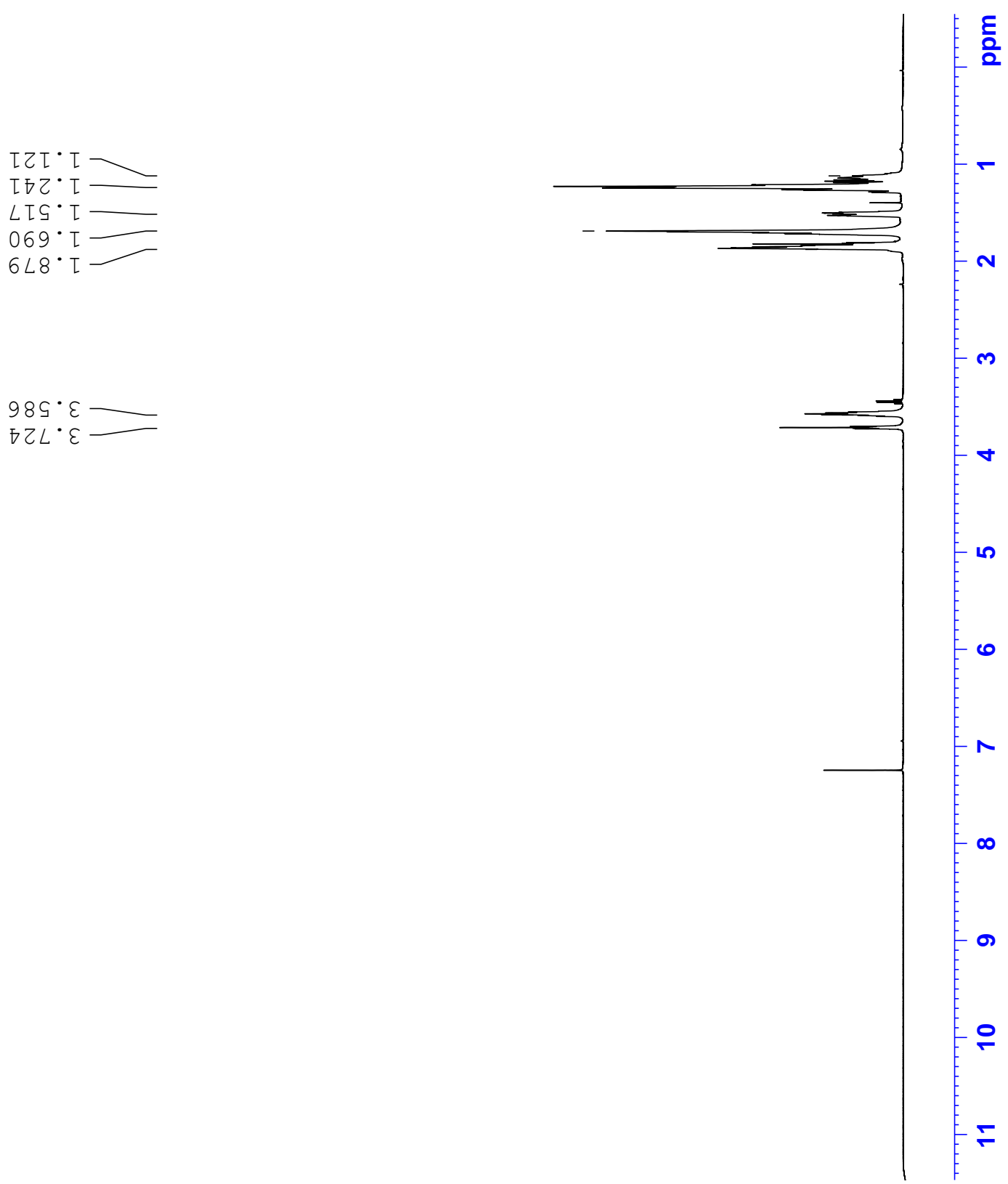

$989^{\circ} \varepsilon$ 


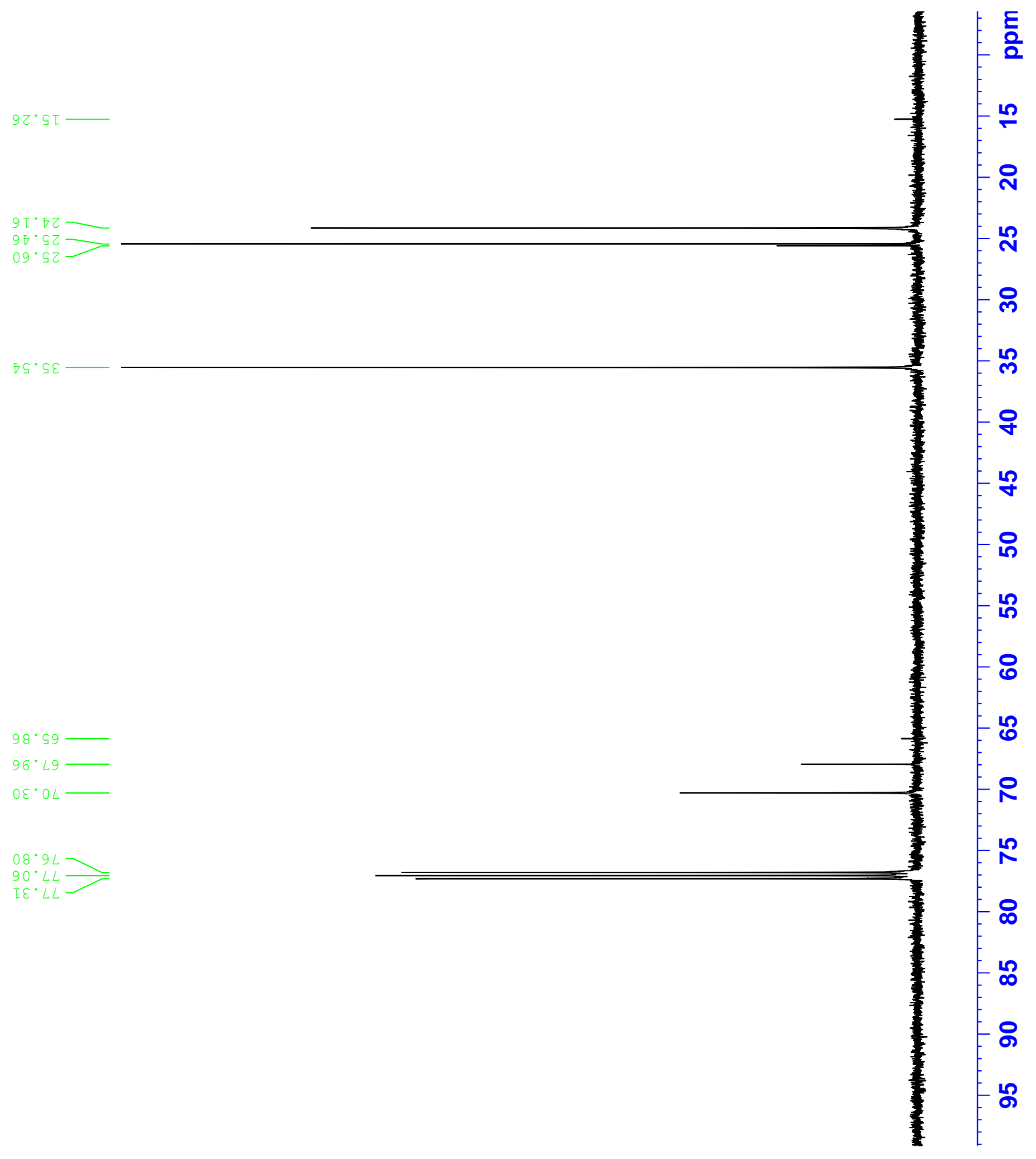


1,5-Decanediol:

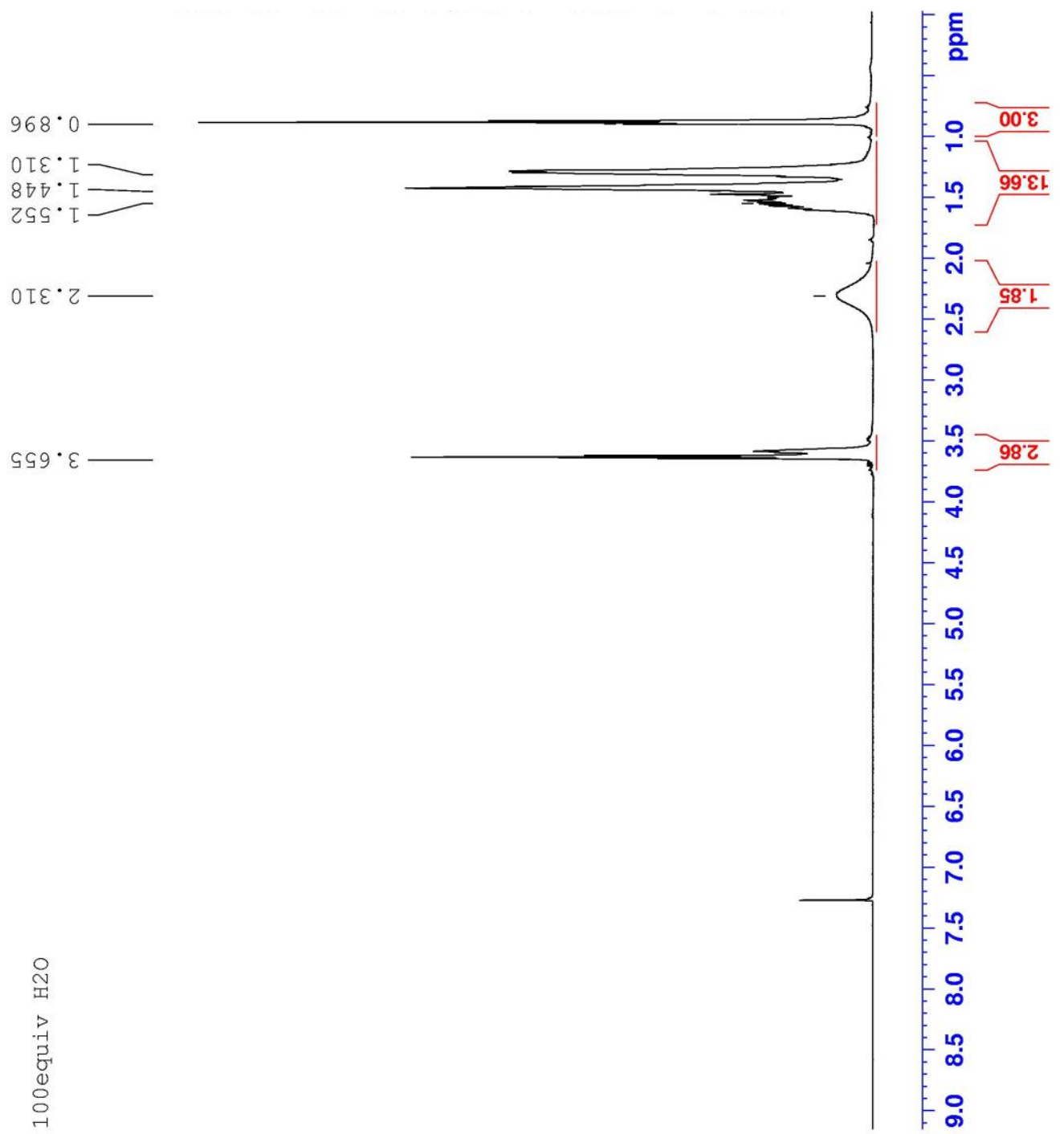




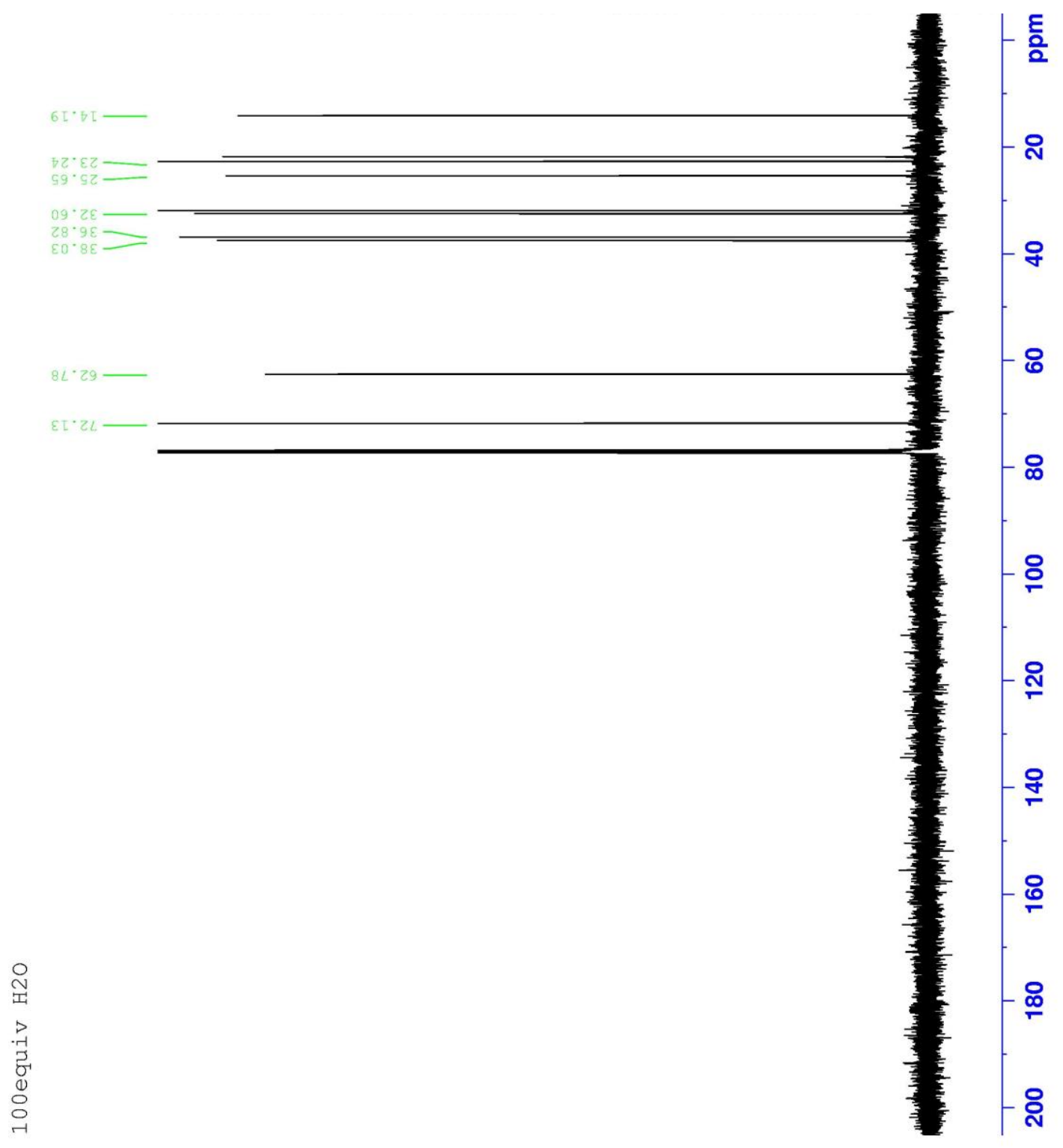


1,1'-Dicyclohexanol:

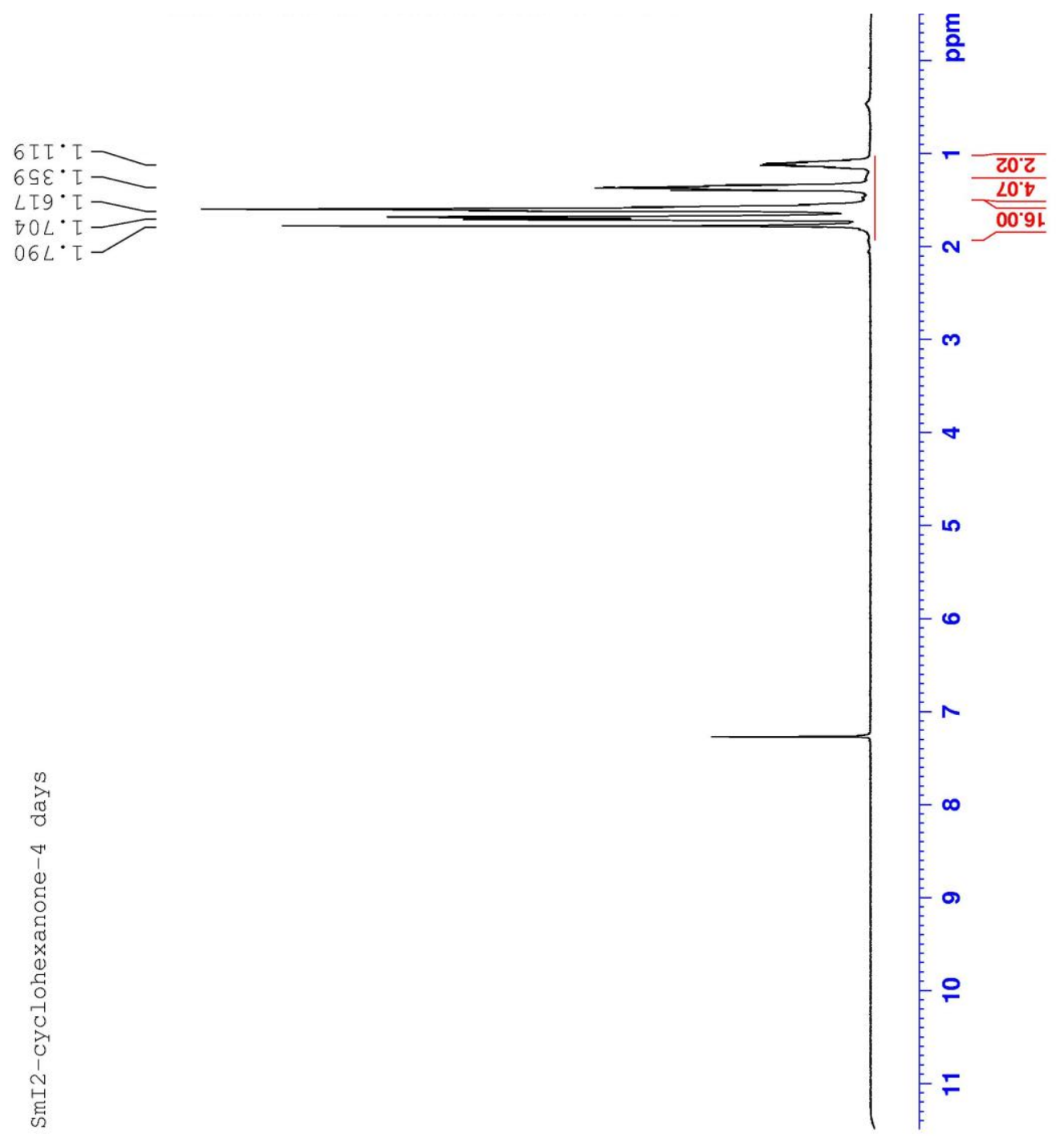


7,8-Tetradecanediol

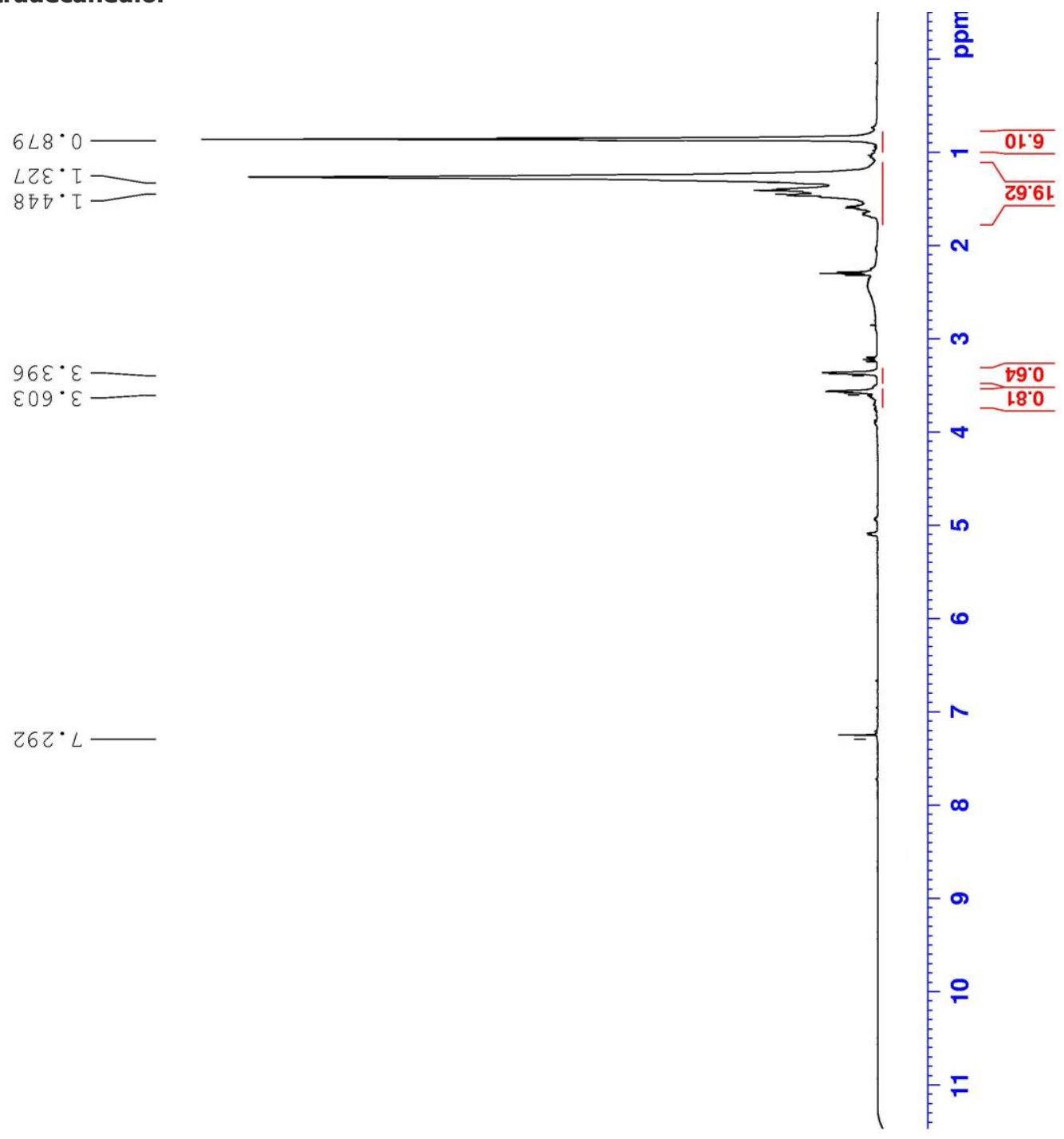




\section{Computational Methods}

Gaussian09(1) programs were used for the calculations with the APF-D(2) hybrid DFT(3) method and the $6-311+g(2 d, p)$ basis set $(4,5)$. Solvation values were calculated using the polarizable continuum model with integral equation formalism. IEFPCM $(6,7)$, with tetrahydrofuran as the solvent. The geometries and frequencies were calculated with uapfd/6-311+g(2d,p) opt $=($ calcfc,tight) int=(ultrafine, acc2e=12) pop=npa. scrf=(iefpcm, solvent=thf) was added to the route section for solvation. Natural-population analysis(8) was obtained by including pop=npa.

1) Gaussian 09, Revision D.01,

M. J. Frisch, G. W. Trucks, H. B. Schlegel, G. E. Scuseria,

M. A. Robb, J. R. Cheeseman, G. Scalmani, V. Barone, B. Mennucci,

G. A. Petersson, H. Nakatsuji, M. Caricato, X. Li, H. P. Hratchian,

A. F. Izmaylov, J. Bloino, G. Zheng, J. L. Sonnenberg, M. Hada,

M. Ehara, K. Toyota, R. Fukuda, J. Hasegawa, M. Ishida, T. Nakajima,

Y. Honda, O. Kitao, H. Nakai, T. Vreven, J. A. Montgomery, Jr.,

J. E. Peralta, F. Ogliaro, M. Bearpark, J. J. Heyd, E. Brothers,

K. N. Kudin, V. N. Staroverov, T. Keith, R. Kobayashi, J. Normand,

K. Raghavachari, A. Rendell, J. C. Burant, S. S. Iyengar, J. Tomasi,

M. Cossi, N. Rega, J. M. Millam, M. Klene, J. E. Knox, J. B. Cross,

V. Bakken, C. Adamo, J. Jaramillo, R. Gomperts, R. E. Stratmann,

O. Yazyev, A. J. Austin, R. Cammi, C. Pomelli, J. W. Ochterski,

R. L. Martin, K. Morokuma, V. G. Zakrzewski, G. A. Voth,

P. Salvador, J. J. Dannenberg, S. Dapprich, A. D. Daniels,

O. Farkas, J. B. Foresman, J. V. Ortiz, J. Cioslowski,

and D. J. Fox, Gaussian, Inc., Wallingford CT, 2013

2)A. Austin, G. Petersson, M. J. Frisch, F. J. Dobek, G. Scalmani, and K. Throssell, "A density functional with spherical atom dispersion terms", J. Chem. Theory and Comput. 8 (2012) 4989.

3) R. G. Parr and W. Yang, Density-functional theory of atoms and molecules (Oxford Univ. Press, Oxford, 1989).

4)A. D. McLean and G. S. Chandler, "Contracted Gaussian-basis sets for molecular calculations. 1. 2nd row atoms, Z=11-18," J. Chem. Phys., 72 (1980) 5639-48

5)K. Raghavachari, J. S. Binkley, R. Seeger, and J. A. Pople, "Self-Consistent Molecular Orbital Methods. 20. Basis set for correlated wave-functions," J. Chem. Phys., 72 (1980) 650-54.

6) E. Cancès, B. Mennucci, and J. Tomasi, "A new integral equation formalism for the polarizable continuum model: Theoretical background and applications to isotropic and anistropic dielectrics," J. Chem. Phys., 107 (1997) 3032-41.

7) J. Tomasi, B. Mennucci, and R. Cammi, "Quantum mechanical continuum solvation models," Chem. Rev., 105 (2005) 2999-3093.

8)A. E. Reed, R. B. Weinstock, and F. Weinhold, "Natural-population analysis," J. Chem. Phys., 83 (1985) 735-46. 
Natural Population Analysis (NPA) Summary:

\begin{tabular}{|c|c|c|c|c|c|c|c|}
\hline \multicolumn{3}{|c|}{ Heptaldehyde } & \multicolumn{2}{|c|}{ Cyclohexanone } & \multicolumn{3}{|c|}{ d-Valerolactone } \\
\hline \multicolumn{3}{|l|}{ Gas } & \multicolumn{2}{|l|}{ Gas } & \multicolumn{3}{|l|}{ Gas } \\
\hline & \multirow[t]{2}{*}{$\mathrm{C}=\mathrm{O}$} & \multirow[t]{2}{*}{-0.533} & \multirow[t]{2}{*}{$\mathrm{C}=\mathrm{O}$} & \multirow[t]{2}{*}{-0.560} & & $C=O$ & -0.582 \\
\hline & & & & & & C-O- & -0.563 \\
\hline & \multirow[t]{2}{*}{$\mathrm{C}-\mathrm{O}^{-}$} & \multirow[t]{2}{*}{-0.740} & \multirow[t]{2}{*}{$\mathrm{C}-\mathrm{O}^{-}$} & \multirow[t]{2}{*}{-0.711} & & $\mathrm{C}^{-} \mathrm{O}^{-}$ & -0.658 \\
\hline & & & & & & C-O- & -0.590 \\
\hline & \multirow[t]{2}{*}{$\Delta \mathrm{C}=\mathrm{O} \rightarrow \mathrm{C}-\mathrm{O}^{-}$} & \multirow[t]{2}{*}{-0.207} & \multirow[t]{2}{*}{$\Delta \mathrm{C}=\mathrm{O} \rightarrow \mathrm{C}-\mathrm{O}^{-}$} & \multirow[t]{2}{*}{-0.151} & & $\Delta \mathrm{C}=\mathrm{O} \rightarrow \mathrm{C}^{-} \mathrm{O}^{-}$ & -0.076 \\
\hline & & & & & & $\Delta \mathrm{C}-\mathrm{O}-\rightarrow \mathrm{C}^{-} \mathrm{O}^{-}$ & -0.027 \\
\hline \multicolumn{3}{|c|}{ iefpcm } & \multicolumn{2}{|l|}{ iefpcm } & \multicolumn{3}{|c|}{ iefpcm } \\
\hline & \multirow[t]{2}{*}{$\mathrm{C}=\mathrm{O}$} & \multirow[t]{2}{*}{-0.580} & \multirow[t]{2}{*}{$\mathrm{C}=\mathrm{O}$} & \multirow[t]{2}{*}{-0.610} & & $\mathrm{C}=\mathrm{O}$ & -0.642 \\
\hline & & & & & & $\mathrm{C}-\mathrm{O}-$ & -0.567 \\
\hline & \multirow[t]{2}{*}{$\mathrm{C}-\mathrm{O}^{-}$} & \multirow[t]{2}{*}{-0.895} & \multirow[t]{2}{*}{${\mathrm{C}-\mathrm{O}^{-}}^{-}$} & \multirow[t]{2}{*}{-0.899} & & $\mathrm{C}-\mathrm{O}^{-}$ & -0.871 \\
\hline & & & & & & $\mathrm{C}-\mathrm{O}-$ & -0.675 \\
\hline & \multirow[t]{2}{*}{$\Delta \mathrm{C}=\mathrm{O} \rightarrow \mathrm{C}-\mathrm{O}^{-}$} & \multirow[t]{2}{*}{-0.315} & \multirow[t]{2}{*}{$\Delta \mathrm{C}=\mathrm{O} \rightarrow \mathrm{C}-\mathrm{O}^{-}$} & \multirow[t]{2}{*}{-0.289} & & $\Delta \mathrm{C}=\mathrm{O} \rightarrow \mathrm{C}^{-} \mathrm{O}^{-}$ & -0.229 \\
\hline & & & & & & $\Delta \mathrm{C}-\mathrm{O}-\rightarrow \mathrm{C}^{-\mathrm{O}^{-}}$ & -0.108 \\
\hline
\end{tabular}




\section{Heptaldehyde (gas)}

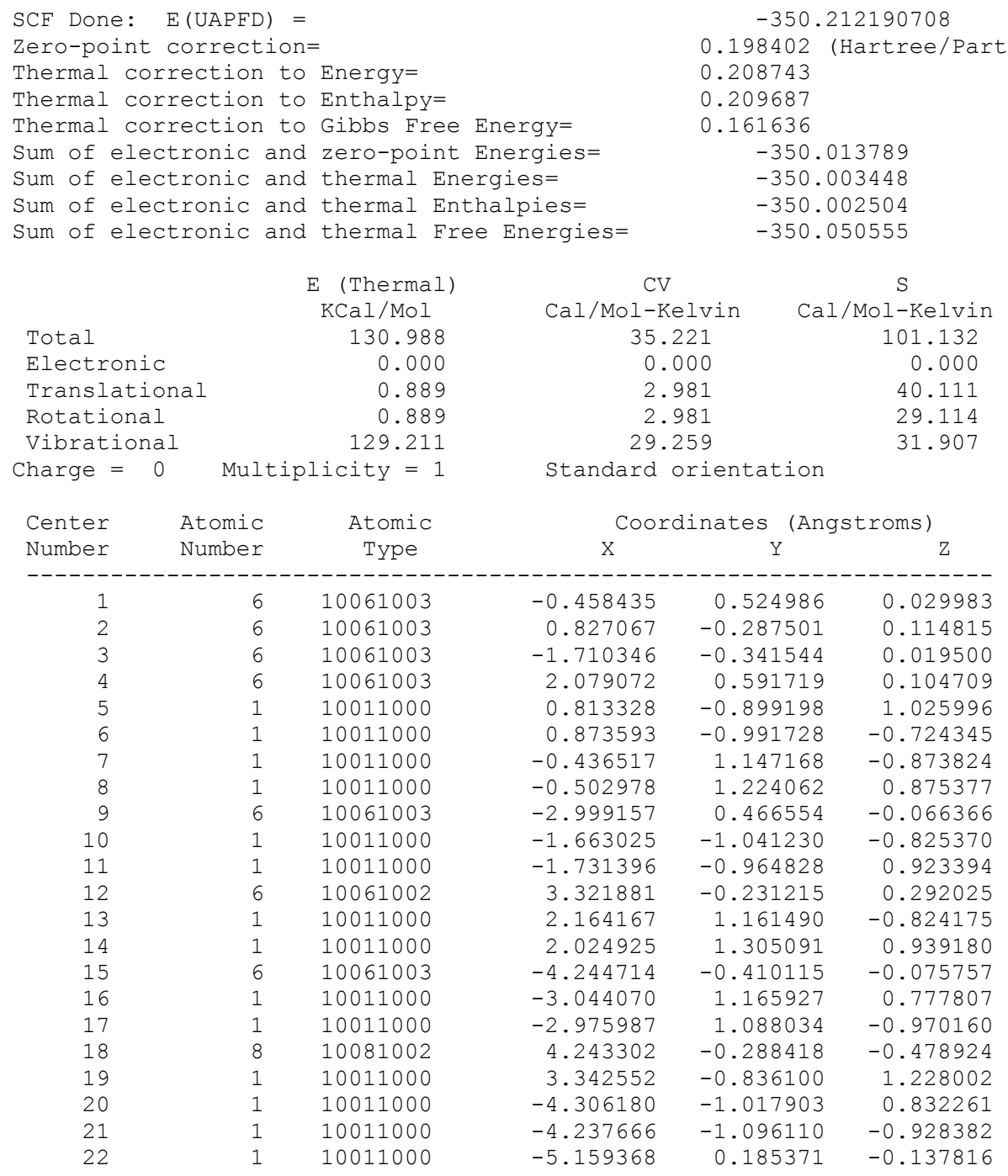

Summary of Natural Population Analysis:

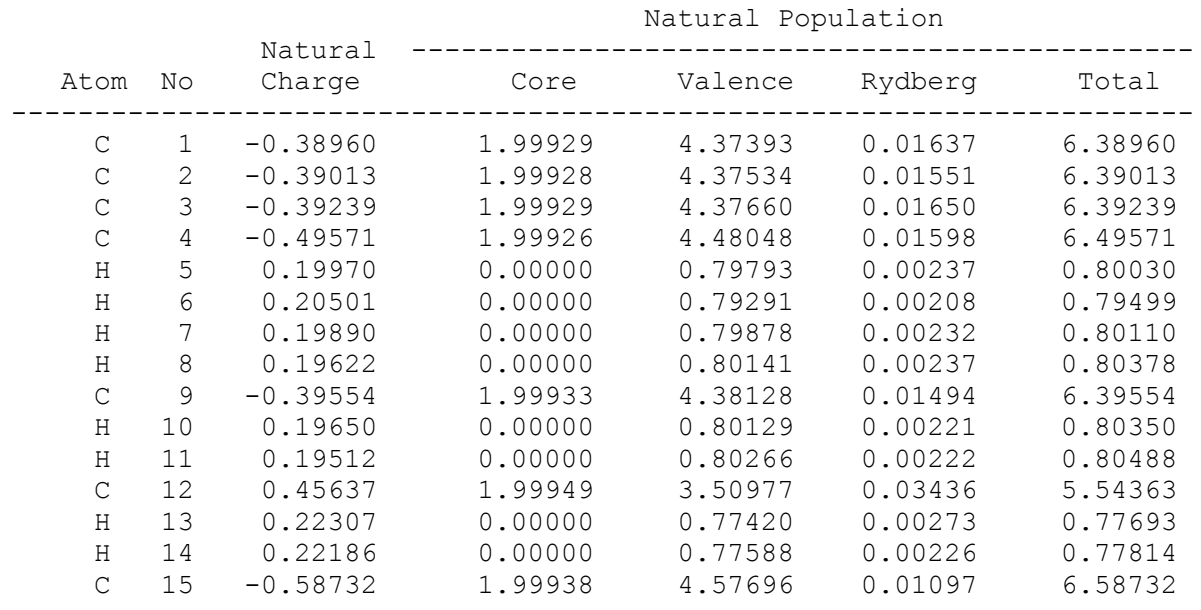




\begin{tabular}{|c|c|c|c|c|c|c|}
\hline $\mathrm{H}$ & 16 & 0.19439 & 0.00000 & 0.80347 & 0.00214 & 0.80561 \\
\hline $\mathrm{H}$ & 17 & 0.19509 & 0.00000 & 0.80277 & 0.00214 & 0.80491 \\
\hline 0 & 18 & -0.53275 & 1.99975 & 6.50271 & 0.03029 & 8.53275 \\
\hline $\mathrm{H}$ & 19 & 0.09785 & 0.00000 & 0.89819 & 0.00396 & 0.90215 \\
\hline $\mathrm{H}$ & 20 & 0.19848 & 0.00000 & 0.79992 & 0.00161 & 0.80152 \\
\hline $\mathrm{H}$ & 21 & 0.19892 & 0.00000 & 0.79948 & 0.00160 & 0.80108 \\
\hline $\mathrm{H}$ & 22 & 0.20595 & 0.00000 & 0.79294 & 0.00111 & 0.79405 \\
\hline & $=====$ & $========$ & $============$ & $===========$ & $=========$ & $=========3$ \\
\hline * Tota & 1 * & 0.00000 & 15.99507 & 47.81889 & 0.18604 & 64.00000 \\
\hline Maximum $\mathrm{F}$ & Porce & & 0.000000 & 0.000015 & YES & \\
\hline RMS & Force & & 0.000000 & 0.000010 & YES & \\
\hline Maximum & Displa & acement & 0.000034 & 0.000060 & YES & \\
\hline RMS & Displa & acement & 0.000008 & 0.000040 & YES & \\
\hline
\end{tabular}

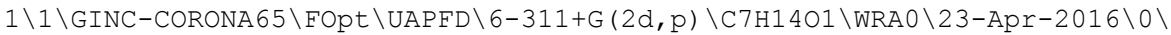
$\backslash \#$ opt $=($ calcfc, tight) freq uapfd $/ 6-311+g(2 d, p)$ pop=npa geom=connectivi ty int=(ultrafine, acc2e=12) \\HALDMM0422A. LOG $-->$ method $5-->$ Hald apfd $0422 \mathrm{a} . g j f \backslash \backslash 0,1 \backslash \mathrm{C},-0.4461364643,0.523548974,0.0276162034 \backslash \mathrm{C}, 0.83745 \overline{4} 8991$ $,-0.2895860463,0.1327466453 \backslash C,-1.699628686,-0.3407556133,0.0275719838 \backslash$ $\mathrm{C}, 2.0911516496,0.5870310421,0.1119740022 \backslash \mathrm{H}, 0.8186890362,-0.8845375877$, $1.0548604021 \backslash \mathrm{H}, 0.8862111052,-1.0090826709,-0.6932287738 \backslash \mathrm{H},-0.419203448$ $1,1.1291038037,-0.8872808978 \backslash \mathrm{H},-0.4929471623,1.2380066389,0.8599269409$ $\backslash \mathrm{C},-2.9865325939,0.4679790919,-0.0785267162 \backslash \mathrm{H},-1.6500435555,-1.0558174$ $267,-0.8041920989 \backslash \mathrm{H},-1.7256962768,-0.947411718,0.9425797762 \backslash \mathrm{C}, 3.331595$ $5729,-0.2346028384,0.3195794081 \backslash \mathrm{H}, 2.1812687295,1.1396112498,-0.8267687$ $592 \backslash \mathrm{H}, 2.0348114471,1.3155999299,0.9330631149 \backslash \mathrm{C},-4.2336944843,-0.406454$ $7822,-0.0772666131 \backslash \mathrm{H},-3.0337064537,1.1827115833,0.7525572011 \backslash \mathrm{H},-2.9583$ $482414,1.0728299872,-0.9933918794 \backslash 0,4.2561726358,-0.307527753,-0.44624$ $76478 \backslash \mathrm{H}, 3.3471466932,-0.8223540636,1.2665068806 \backslash \mathrm{H},-4.3001657703,-0.997$ $4691131,0.8414085819 \backslash \mathrm{H},-4.2243229806,-1.1078969194,-0.9172066258 \backslash \mathrm{H},-5$. $1469486514,0.1894582317,-0.1540851285 \backslash \backslash$ Version=EM64L-G09RevD.01 \State $=$ $1-\mathrm{A} \backslash \mathrm{HF}=-350.2121907 \backslash \mathrm{S} 2=0 . \backslash \mathrm{S} 2-1=0 . \backslash \mathrm{S} 2 \mathrm{~A}=0 . \backslash \mathrm{RMSD}=6.130 \mathrm{e}-09 \backslash \mathrm{RMSF}=1.983 e-07$ $\backslash$ Dipole $=-1.134177,0.1900565,0.5469986 \backslash$ Quadrupole $=-9.231654,4.2754078,4$ $.9562462,1.206771,4.3533655,-0.1891487 \backslash P G=C 01 \quad[X(C 7 H 1401)] \backslash \backslash @$

$\begin{array}{lllll}\text { Maximum } & \text { Force } & 0.000000 & 0.000015 & \text { YES } \\ \text { RMS } & \text { Force } & 0.000000 & 0.000010 & \text { YES } \\ \text { Maximum } & \text { Displacement } & 0.000011 & 0.000060 & \text { YES } \\ \text { RMS } & \text { Displacement } & 0.000003 & 0.000040 & \text { YES }\end{array}$

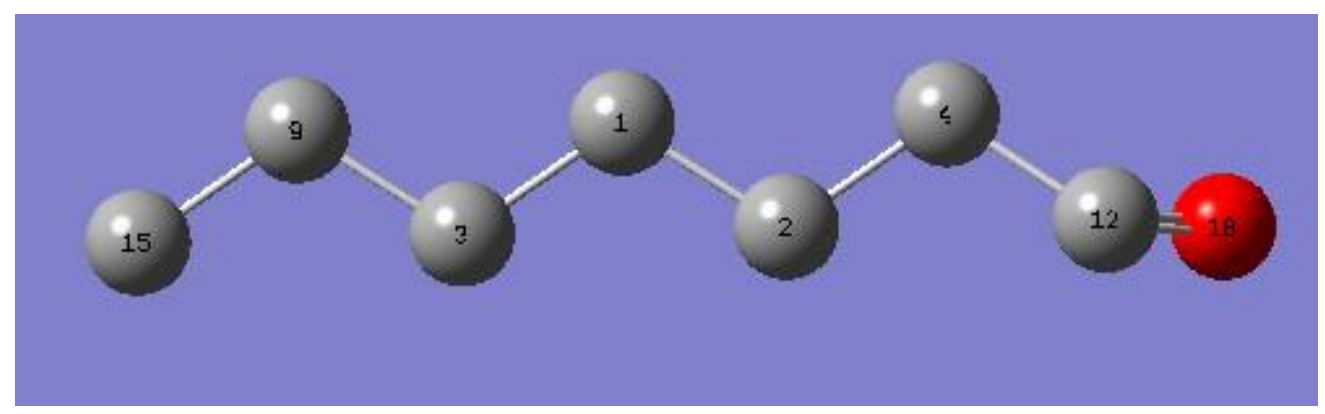




\section{Heptaldehyde (iefpcm)}

SCF Done: $\mathrm{E}$ (UAPFD) =

Zero-point correction=

Thermal correction to Energy=

Thermal correction to Enthalpy=

Thermal correction to Gibbs Free Energy=

Sum of electronic and zero-point Energies=

Sum of electronic and thermal Energies=

Sum of electronic and thermal Enthalpies=

Sum of electronic and thermal Free Energies=
$-350.217382031$

0.198189 (Hartree/Particle)

0.208536

0.209480

0.161407

$-350.019193$

$-350.008846$

$-350.007902$

$-350.055975$

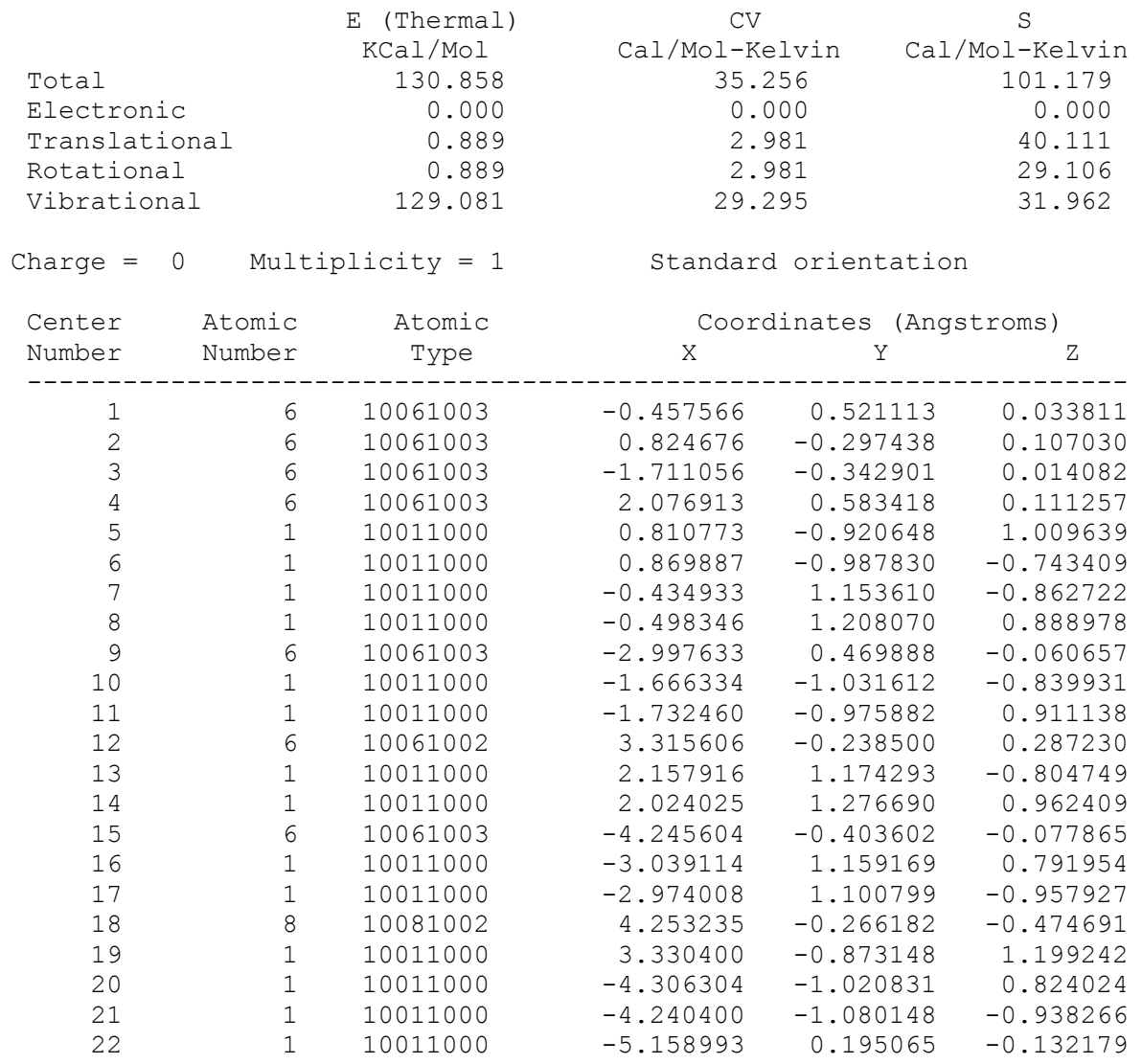

Summary of Natural Population Analysis:

Natural Population

\begin{tabular}{|c|c|c|c|c|c|c|}
\hline Atom & No & $\begin{array}{l}\text { Natural } \\
\text { Charge }\end{array}$ & Core & Valence & Rydberg & Total \\
\hline & & & & & -1 & - \\
\hline C & 1 & -0.39065 & 1.99929 & 4.37495 & 0.01641 & 6.39065 \\
\hline C & 2 & -0.38988 & 1.99927 & 4.37509 & 0.01551 & 6.38988 \\
\hline C & 3 & -0.39316 & 1.99929 & 4.37736 & 0.01651 & 6.39316 \\
\hline C & 4 & -0.49938 & 1.99925 & 4.48399 & 0.01614 & 6.49938 \\
\hline $\mathrm{H}$ & 5 & 0.20461 & 0.00000 & 0.79314 & 0.00224 & 0.79539 \\
\hline $\mathrm{H}$ & 6 & 0.20579 & 0.00000 & 0.79216 & 0.00204 & 0.79421 \\
\hline $\mathrm{H}$ & 7 & 0.20000 & 0.00000 & 0.79772 & 0.00228 & 0.80000 \\
\hline $\mathrm{H}$ & 8 & 0.19882 & 0.00000 & 0.79886 & 0.00231 & 0.80118 \\
\hline C & 9 & -0.39622 & 1.99933 & 4.38191 & 0.01498 & 6.39622 \\
\hline $\mathrm{H}$ & 10 & 0.19710 & 0.00000 & 0.80071 & 0.00219 & 0.80290 \\
\hline $\mathrm{H}$ & 11 & 0.19635 & 0.00000 & 0.80146 & 0.00219 & 0.80365 \\
\hline C & 12 & 0.47243 & 1.99949 & 3.49444 & 0.03364 & 5.52757 \\
\hline $\mathrm{H}$ & 13 & 0.22298 & 0.00000 & 0.77440 & 0.00262 & 0.77702 \\
\hline $\mathrm{H}$ & 14 & 0.23372 & 0.00000 & 0.76412 & 0.00216 & 0.76628 \\
\hline C & 15 & -0.58968 & 1.99938 & 4.57938 & 0.01091 & 6.58968 \\
\hline $\mathrm{H}$ & 16 & 0.19515 & 0.00000 & 0.80271 & 0.00214 & 0.80485 \\
\hline
\end{tabular}




\begin{tabular}{|c|c|c|c|c|c|c|}
\hline $\mathrm{H}$ & 17 & 0.19551 & 0.00000 & 0.80235 & 0.00214 & 0.80449 \\
\hline 0 & 18 & -0.57983 & 1.99975 & 6.55056 & 0.02952 & 8.57983 \\
\hline $\mathrm{H}$ & 19 & 0.11268 & 0.00000 & 0.88380 & 0.00352 & 0.88732 \\
\hline $\mathrm{H}$ & 20 & 0.19891 & 0.00000 & 0.79947 & 0.00161 & 0.80109 \\
\hline $\mathrm{H}$ & 21 & 0.19914 & 0.00000 & 0.79925 & 0.00161 & 0.80086 \\
\hline $\mathrm{H}$ & 22 & 0.20560 & 0.00000 & 0.79328 & 0.00111 & 0.79440 \\
\hline$:======$ & $===$ & $=======$ & $=======0=$ & $=======$ & $=======$ & $========$ \\
\hline * Total & 1 * & 0.00000 & 15.99506 & 47.82114 & 0.18381 & 64.00000 \\
\hline Maximum Fo & orce & & 0.000000 & 0.000015 & YES & \\
\hline RMS $\quad \mathrm{F}$ & For & & 0.000000 & 0.000010 & YES & \\
\hline Maximum D & Dist & acement & 0.000033 & 0.000060 & YES & \\
\hline RMS $\quad D$ & Disk & acement & 0.000009 & 0.000040 & YES & \\
\hline
\end{tabular}

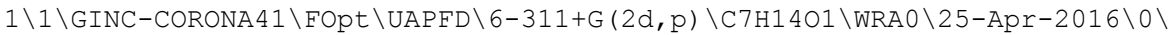
$\backslash \#$ opt $=($ calcf, tight $)$ freq uapfd/6-311+g(2d,p) scrf=(iefpcm, solvent=th

f) pop=npa geom=connectivity int=(ultrafine, acc2e=12) \\1) Heptaaldehyde Z clean $->$ opt=(calcfc,tight) uff scrf $=($ iefpcm, solvent=thf) int=ultr afine fname=HaldMM pcm0425.gjf 2) HaldMM pcm0425.log $->$ (M60) \# opt $=($ ca lcfc,tight) freq uapfd/6-311+g (2d,p) scrfe(iefpcm, solvent=thf) pop=npa geom=connectivity int=(ultrafine, acc2e=12) fname=Hald $p c m 0425 . g j f \backslash \backslash 0$, $1 \backslash \mathrm{C},-0.4450445096,0.5217577533,0.0242463564 \backslash \mathrm{C}, 0.836341 \overline{2} 845,-0.29443430$ $84,0.131066228 \backslash \mathrm{C},-1.6989479625,-0.3418753078,0.0276377686 \backslash \mathrm{C}, 2.08907577$ $76,0.5855100832,0.1116087141 \backslash \mathrm{H}, 0.8177851911,-0.8869110701,1.0540576285$ $\backslash \mathrm{H}, 0.885166814,-1.0130739503,-0.6954354487 \backslash \mathrm{H},-0.4177805277,1.123716675$ $8,-0.8929440936 \backslash \mathrm{H},-0.4894633835,1.2371206828,0.8556099856 \backslash \mathrm{C},-2.9846661$ $699,0.4685086967,-0.0805281868 \backslash \mathrm{H},-1.6505941014,-1.0589542787,-0.802494$ $3664 \backslash \mathrm{H},-1.7249856173,-0.9443004103,0.9453735184 \backslash \mathrm{C}, 3.3264243142,-0.2305$ $701273,0.3210296447 \backslash \mathrm{H}, 2.1747765734,1.1451891194,-0.8233646768 \backslash \mathrm{H}, 2.0325$ $722063,1.3070544403,0.938689394 \backslash \mathrm{C},-4.2330681642,-0.4045119097,-0.07427$ $17199 \backslash \mathrm{H},-3.0297715961,1.1861092665,0.7481994761 \backslash \mathrm{H},-2.9564079581,1.0688$ $579473,-0.9983964923 \backslash 0,4.267637919,-0.2842924173,-0.4350591046 \backslash \mathrm{H}, 3.336$ $5134144,-0.8341750351,1.2539404079 \backslash \mathrm{H},-4.2984148124,-0.9910144164,0.847$ $5767402 \backslash \mathrm{H},-4.2241944069,-1.1096305281,-0.9113858804 \backslash \mathrm{H},-5.1458282847,0$. $192393094,-0.1530438931 \backslash \backslash$ Version=EM64L-G0 9RevD.01 \State=1-A \HF=-350.21 $7382 \backslash \mathrm{S} 2=0 . \backslash \mathrm{S} 2-1=0 . \backslash \mathrm{S} 2 \mathrm{~A}=0 . \backslash \mathrm{RMSD}=8.443 \mathrm{e}-09 \backslash \mathrm{RMSF}=8.684 \mathrm{e}-07 \backslash \mathrm{Dipole}=-1.3252$ $079,0.2039703,0.6963942 \backslash$ Quadrupole $=-11.2893632,5.2678976,6.0214656,1.1$ $939338,5.2009086,-0.2108157 \backslash P G=C 01 \quad[X(C 7 H 1401)] \backslash \backslash @$

$\begin{array}{lllll}\text { Maximum } & \text { Force } & 0.000001 & 0.000015 & \text { YES } \\ \text { RMS } & \text { Force } & 0.000000 & 0.000010 & \text { YES } \\ \text { Maximum } & \text { Displacement } & 0.000039 & 0.000060 & \text { YES } \\ \text { RMS } & \text { Displacement } & 0.000009 & 0.000040 & \text { YES }\end{array}$

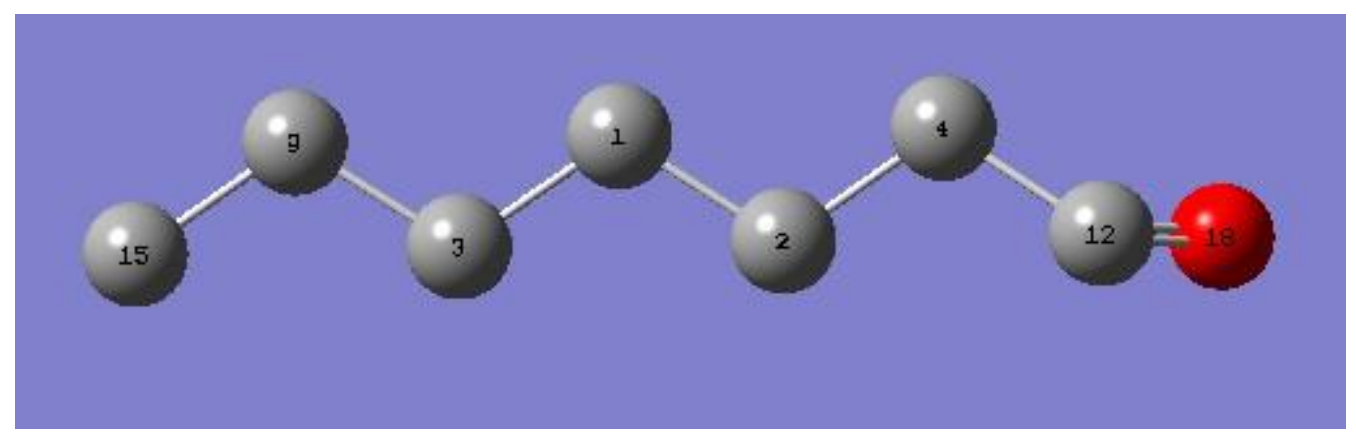




\section{Heptaldehyde radical ion (gas)}

Zero-point correction=

Thermal correction to Energy=

Thermal correction to Enthalpy=

Thermal correction to Gibbs Free Energy=

Sum of electronic and zero-point Energies=

Sum of electronic and thermal Energies=

Sum of electronic and thermal Enthalpies=

Sum of electronic and thermal Free Energies=
$\mathrm{SCF}$ Done: $\mathrm{E}(\mathrm{UAPFD})=$

-350.185654575
$0.194757 \quad$ (Hartree/Particle)
0.205189
0.206133
0.157577
-349.990897
-349.980465
-349.979521
-350.028078

CV

$\mathrm{KCal} / \mathrm{Mol}$

Total

Electronic

Translational

Rotational

Vibrational

128.758

0.000

0.889

0.889

126.981

Charge $=-1$ Multiplicity $=2$

\begin{tabular}{|c|c|c|c|c|c|}
\hline \multirow{2}{*}{$\begin{array}{l}\text { Center } \\
\text { Number }\end{array}$} & \multirow{2}{*}{$\begin{array}{l}\text { Atomic } \\
\text { Number }\end{array}$} & \multirow{2}{*}{$\begin{array}{l}\text { Atomic } \\
\text { Type }\end{array}$} & \multicolumn{3}{|c|}{ Coordinates (Angstroms) } \\
\hline & & & $\mathrm{X}$ & $\mathrm{Y}$ & $\mathrm{Z}$ \\
\hline--1 & & & --ー-ー-ー-ー- & ---------1 & ---------- \\
\hline 1 & 6 & 0 & -0.427038 & 0.569417 & -0.092483 \\
\hline 2 & 6 & 0 & 0.896239 & -0.113346 & 0.219434 \\
\hline 3 & 6 & 0 & -1.650638 & -0.309331 & 0.141878 \\
\hline 4 & 6 & 0 & 2.118889 & 0.767306 & -0.070562 \\
\hline 5 & 1 & 0 & 0.920023 & -0.413102 & 1.275372 \\
\hline 6 & 1 & 0 & 0.997833 & -1.037049 & -0.361048 \\
\hline 7 & 1 & 0 & -0.424171 & 0.911633 & -1.136700 \\
\hline 8 & 1 & 0 & -0.520024 & 1.481344 & 0.516136 \\
\hline 9 & 6 & 0 & -2.962510 & 0.387796 & -0.196192 \\
\hline 10 & 1 & 0 & -1.561264 & -1.226506 & -0.455182 \\
\hline 11 & 1 & 0 & -1.670895 & -0.639894 & 1.188582 \\
\hline 12 & 6 & 0 & 3.434431 & 0.101654 & 0.230631 \\
\hline 13 & 1 & 0 & 2.072050 & 1.034157 & -1.148449 \\
\hline 14 & 1 & 0 & 2.042378 & 1.707696 & 0.493146 \\
\hline 15 & 6 & 0 & -4.188370 & -0.481486 & 0.049849 \\
\hline 16 & 1 & 0 & -3.042500 & 1.311903 & 0.391313 \\
\hline 17 & 1 & 0 & -2.940392 & 0.707543 & -1.246198 \\
\hline 18 & 8 & 0 & 3.772211 & -0.987722 & -0.301353 \\
\hline 19 & 1 & 0 & 4.152217 & 0.679477 & 0.847177 \\
\hline 20 & 1 & 0 & -4.254887 & -0.785383 & 1.100274 \\
\hline 21 & 1 & 0 & -4.152549 & -1.397749 & -0.549384 \\
\hline 22 & 1 & 0 & -5.121531 & 0.035651 & -0.199550 \\
\hline
\end{tabular}

102.196

1.377

40.111

29.273

31.434
30.266
standard orientation

$\begin{array}{rr}0.000 & 1.377 \\ 2.981 & 40.111 \\ 2.981 & 29.273 \\ 30.266 & 31.434\end{array}$

Summary of Natural Population Analysis:

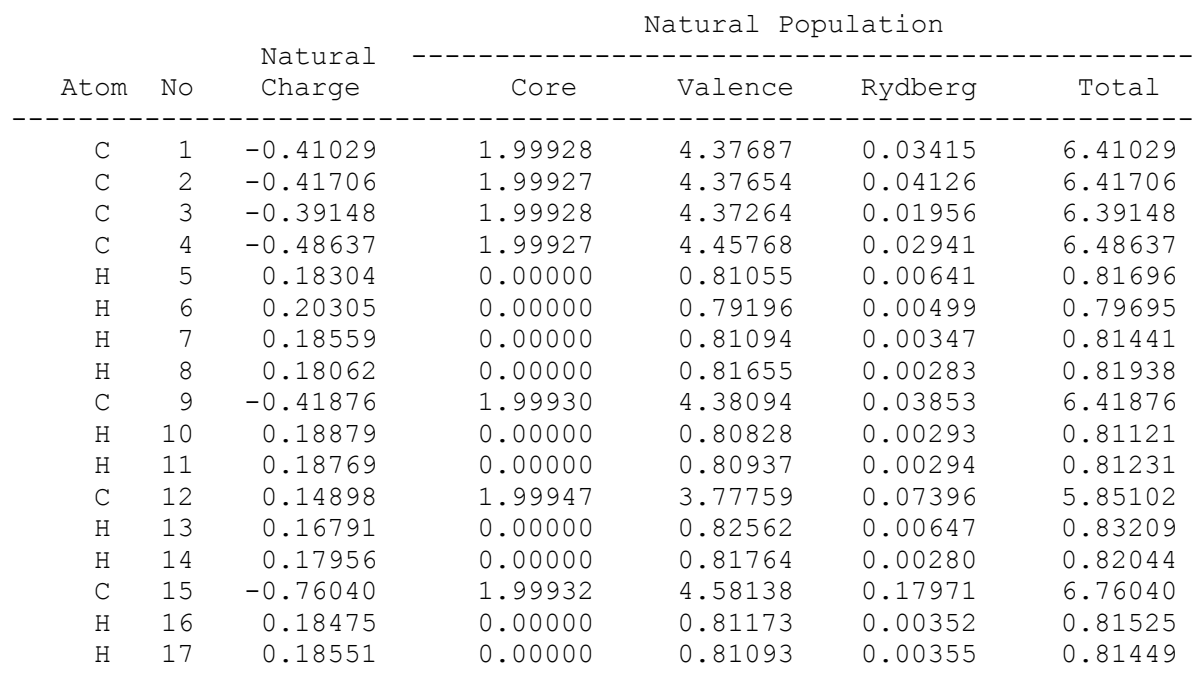




$\begin{array}{rrrrrrr}\mathrm{O} & 18 & -0.73995 & 1.99979 & 6.70263 & 0.03752 & 8.73995 \\ \mathrm{H} & 19 & 0.08041 & 0.00000 & 0.91451 & 0.00507 & 0.91959 \\ \mathrm{H} & 20 & 0.18794 & 0.00000 & 0.80870 & 0.00336 & 0.81206 \\ \mathrm{H} & 21 & 0.18824 & 0.00000 & 0.80824 & 0.00353 & 0.81176 \\ \mathrm{H} & 22 & 0.17225 & 0.00000 & 0.80207 & 0.02568 & 0.82775 \\ ===================================================================0 \\ \text { * Total * } & -1.00000 & 15.99497 & 48.47337 & 0.53166 & 65.00000\end{array}$

$\begin{array}{llccc}\text { Maximum } & \text { Force } & 0.000002 & 0.000015 & \text { YES } \\ \text { RMS } & \text { Force } & 0.000000 & 0.000010 & \text { YES } \\ \text { Maximum } & \text { Displacement } & 0.000055 & 0.000060 & \text { YES } \\ \text { RMS } & \text { Displacement } & 0.000015 & 0.000040 & \text { YES }\end{array}$

$1 \backslash 1 \backslash$ GINC-CORONA55 $\backslash$ FOpt \UAPFD $\backslash 6-311+\mathrm{G}(2 \mathrm{~d}, \mathrm{p}) \backslash \mathrm{C} 7 \mathrm{H} 1401(1-, 2) \backslash$ WRA $\backslash 24-$ Apr -2 $016 \backslash 0 \backslash \backslash \#$ opt=(calcfc,tight) freq uapfd/6-311+g $(2 d, p)$ pop=npa geom=conn ectivity int=(ultrafine, acc2e=12) \\HaldMM0320.gjf -->mod to radical io

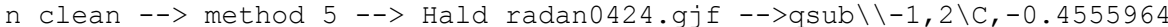
$127,0.4909621,0.12 \overline{8} 5066619 \backslash \mathrm{C}, \overline{0} .8362240308,-0.3112533809,0.1751007481 \backslash \mathrm{C}$ $,-1.7121965761,-0.361236161,-0.0094579094 \backslash \mathrm{C}, 2.0935470564,0.5639841513$, $0.262384944 \backslash \mathrm{H}, 0.8200691101,-0.9921755936,1.0362094651 \backslash \mathrm{H}, 0.9258366652,-$ $0.9465758856,-0.7130669567 \backslash \mathrm{H},-0.4118346199,1.205554338,-0.7051313749 \backslash \mathrm{H}$ $,-0.5377897359,1.1043253988,1.0382885191 \backslash \mathrm{C},-2.991417502,0.4637742034,-$ $0.0745454852 \backslash \mathrm{H},-1.6341097304,-0.983714585,-0.9106237281 \backslash \mathrm{H},-1.773042879$ $5,-1.0653258536,0.830677648 \backslash \mathrm{C}, 3.3784816856,-0.218028577,0.3055050099 \backslash \mathrm{H}$ $, 2.0862807085,1.2238208981,-0.6319337082 \backslash \mathrm{H}, 2.0299995537,1.2201216721,1$ $.1418154572 \backslash \mathrm{C},-4.2503201294,-0.3840522845,-0.1981317368 \backslash \mathrm{H},-3.059636032$ $8,1.0959295318,0.8205861291 \backslash \mathrm{H},-2.9289533891,1.159063508,-0.921841668 \backslash 0$ $, 3.6975111185,-1.034248956,-0.5977380238 \backslash \mathrm{H}, 4.0957128305,0.0515334442,1$ $.106841326 \backslash \mathrm{H},-4.3566638771,-1.0630987334,0.6549558451 \backslash \mathrm{H},-4.2256470391$, $-1.0027279739,-1.1017537008 \backslash \mathrm{H},-5.1593368454,0.2258287389,-0.2445394516$

\\Version=EM64L-G09RevD.01 \State $=2-\mathrm{A} \backslash \mathrm{HF}=-350.1856546 \backslash \mathrm{S} 2=0.75224 \backslash \mathrm{S} 2-1=0$ $. \backslash \mathrm{S} 2 \mathrm{~A}=0.750004 \backslash \mathrm{RMSD}=5.036 \mathrm{e}-09 \backslash \mathrm{RMSF}=5.618 \mathrm{e}-07 \backslash \mathrm{Dipole}=-1.668864,0.818711$ $1,0.4381844 \backslash$ Quadrupole $=-38.9621637,18.1992119,20.7629517,7.2358584,1.8$ $581908,-0.8367359 \backslash \mathrm{PG}=\mathrm{C} 01 \quad[\mathrm{X}(\mathrm{C} 7 \mathrm{H} 1401)] \backslash \backslash @$

$\begin{array}{lllll}\text { Maximum } & \text { Force } & 0.000002 & 0.000015 & \text { YES } \\ \text { RMS } & \text { Force } & 0.000000 & 0.000010 & \text { YES } \\ \text { Maximum } & \text { Displacement } & 0.000051 & 0.000060 & \text { YES } \\ \text { RMS } & \text { Displacement } & 0.000014 & 0.000040 & \text { YES }\end{array}$

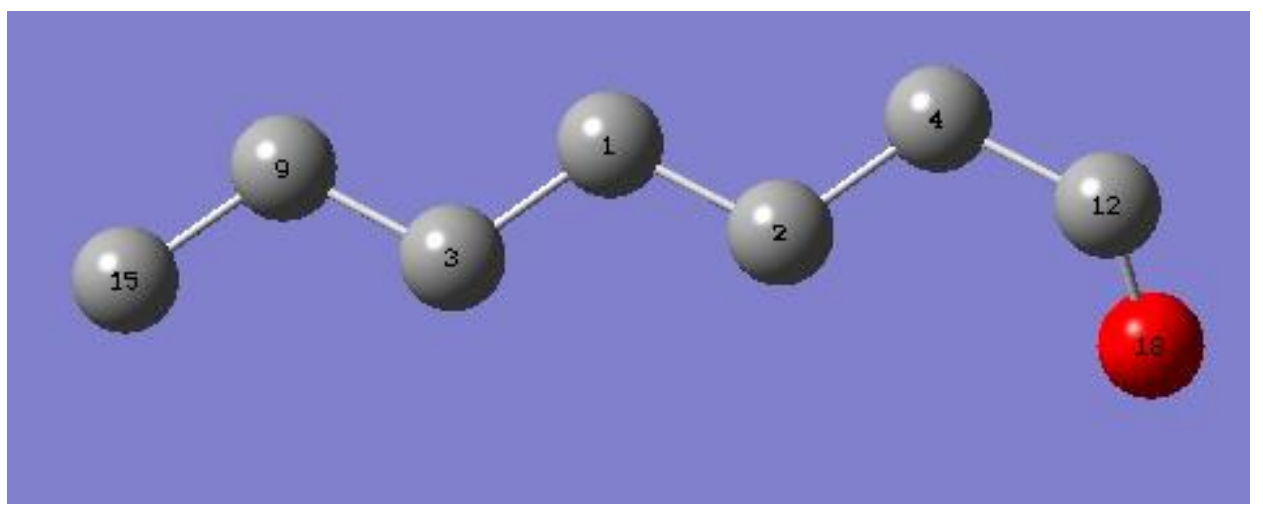




\section{Heptaldehyde radical ion (iefpcm)}

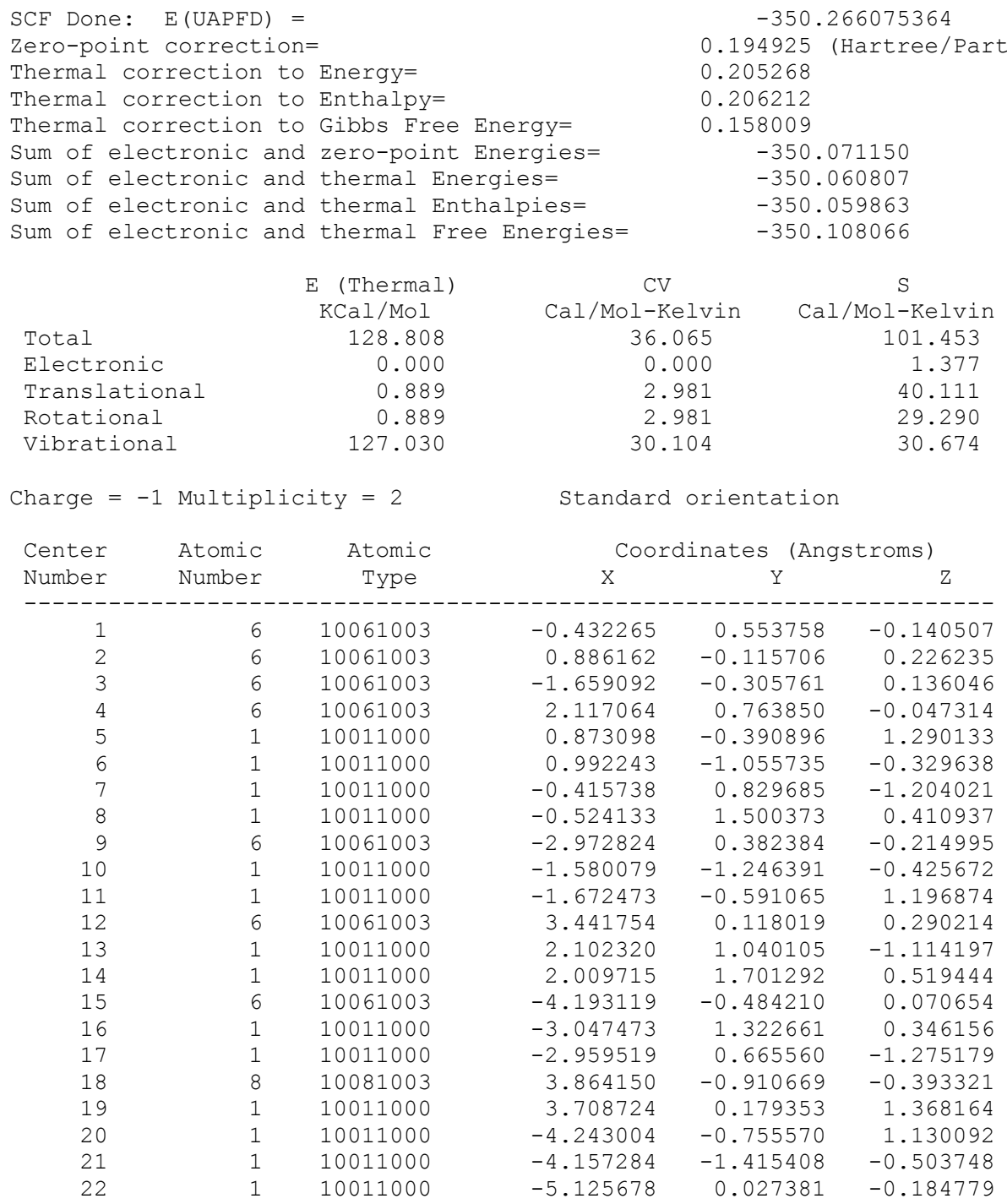

Summary of Natural Population Analysis:

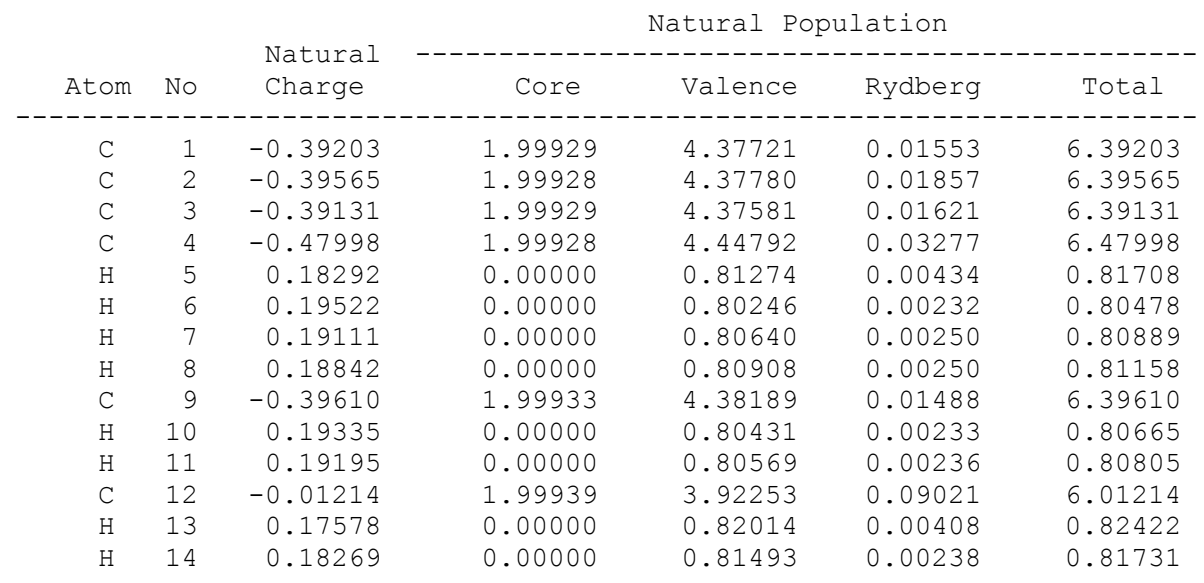




$\begin{array}{crrcccl}\text { C } & 15 & -0.58975 & 1.99939 & 4.57951 & 0.01085 & 6.58975 \\ \mathrm{H} & 16 & 0.19236 & 0.00000 & 0.80540 & 0.00224 & 0.80764 \\ \mathrm{H} & 17 & 0.19306 & 0.00000 & 0.80470 & 0.00223 & 0.80694 \\ \mathrm{O} & 18 & -0.89492 & 1.99982 & 6.86575 & 0.02935 & 8.89492 \\ \mathrm{H} & 19 & 0.06699 & 0.00000 & 0.92754 & 0.00547 & 0.93301 \\ \mathrm{H} & 20 & 0.19757 & 0.00000 & 0.80075 & 0.00168 & 0.80243 \\ \mathrm{H} & 21 & 0.19802 & 0.00000 & 0.80031 & 0.00167 & 0.80198 \\ \mathrm{H} & 22 & 0.20243 & 0.00000 & 0.79641 & 0.00117 & 0.79757 \\ ======================================================= \\ \text { * Total * }-1.00000 & 15.99507 & 48.73929 & 0.26564 & 65.00000 \\ \text { Maximum Force } & & & & \end{array}$

$1 \backslash 1 \backslash$ GINC-CORONA 65 \FOpt \UAPFD \6-311+G $(2 \mathrm{~d}, \mathrm{p}) \backslash \mathrm{C} 7 \mathrm{H} 14 \mathrm{O}(1-, 2) \backslash$ WRA $\backslash 25-A p r-2$ $016 \backslash 0 \backslash \backslash \#$ opt=(calcfC, tight) freq uapfd/6-311+g $(2 \mathrm{~d}, \mathrm{p})$ scrf=(iefpcm, solv ent=thf) pop=npa geom=connectivity int=(ultrafine, acc $2 e=12) \backslash \backslash$ HALDMM 03 20.gjf mod to radical anion clean --> opt=(calcfc,tight) uff scrf=(ief pcm, solvent=thf) int=ultrafine fname=Hald radanMMpcm0425 $0425-->$ Hald _radanMMpcm0425.log --> method_6 --> Hald_radan_pcm_m6_gjf0425 --> qsu $\overline{\mathrm{b}} \backslash \backslash-1,2 \backslash \mathrm{C},-0.4168813465,0.5455 \overline{5} 52835,-0.1 \overline{4} 52319 \overline{8} 68 \backslash \overline{\mathrm{C}}, 0.8969960899,-0.1$ $243787592,0.2366667007 \backslash C,-1.6486859429,-0.3044670454,0.1384539489 \backslash \mathrm{C}, 2$. $1329525304,0.7457044543,-0.0442861413 \backslash \mathrm{H}, 0.8782683628,-0.3841698559,1.3$ $043446114 \backslash \mathrm{H}, 1.0011810449,-1.0727682573,-0.3051859972 \backslash \mathrm{H},-0.3946890228,0$ $.8060748445,-1.2125222906 \backslash \mathrm{H},-0.506806313,1.500404316,0.3921575779 \backslash \mathrm{C},-2$ $.9578496044,0.3843511414,-0.2279933854 \backslash \mathrm{H},-1.5715462502,-1.253423183,-0$ $.4093488783 \backslash \mathrm{H},-1.6677628842,-0.5744148097,1.2032064635 \backslash \mathrm{C}, 3.4533197352$, $0.0989529126,0.3080879355 \backslash \mathrm{H}, 2.1238898125,1.0066414541,-1.1150822437 \backslash \mathrm{H}$, $2.0274415432,1.6916706839,0.5084816971 \backslash \mathrm{C},-4.1831926078,-0.4726442858,0$ $.0649163005 \backslash \mathrm{H},-3.0306248311,1.3329259125,0.3192653329 \backslash \mathrm{H},-2.9388615676$, $0.6521800708,-1.2920721358 \backslash 0,3.8739489072,-0.941320105,-0.3588003874 \backslash \mathrm{H}$ $, 3.7160693587,0.174612463,1.3861648178 \backslash \mathrm{H},-4.2387045451,-0.7285082388,1$ $.1279244392 \backslash \mathrm{H},-4.1491348989,-1.4121612276,-0.4958866995 \backslash \mathrm{H},-5.112379570$ $3,0.0393322311,-0.2017846793 \backslash \backslash$ Version $=E M 64 \mathrm{~L}-\mathrm{G} 0$ 9RevD.01 $\backslash$ State $=2-\mathrm{A} \backslash \mathrm{HF}=-3$ $50.2660754 \backslash \mathrm{S} 2=0.754466 \backslash \mathrm{S} 2-1=0 . \backslash \mathrm{S} 2 \mathrm{~A}=0.750012 \backslash \mathrm{RMSD}=8.327 \mathrm{e}-09 \backslash \mathrm{RMSF}=9.850 \mathrm{e}$ $-06 \backslash \mathrm{Dipole}=-7.0336493,0.9516431,0.4215233 \backslash$ Quadrupole $=-35.0876847,15.53$ $35897,19.554095,6.7716466,3.4162885,-0.771935 \backslash \mathrm{PG}=\mathrm{C} 01 \quad[\mathrm{X}(\mathrm{C} 7 \mathrm{H} 14 \mathrm{O} 1)] \backslash \backslash \mathrm{Q}$

Maximum Force $0.000000 \quad 0.000015$ YES

RMS Force $0.000000 \quad 0.000010$ YES

Maximum Displacement $0.000003 \quad 0.000060$ YES

RMS Displacement $0.000001 \quad 0.000040$ YES

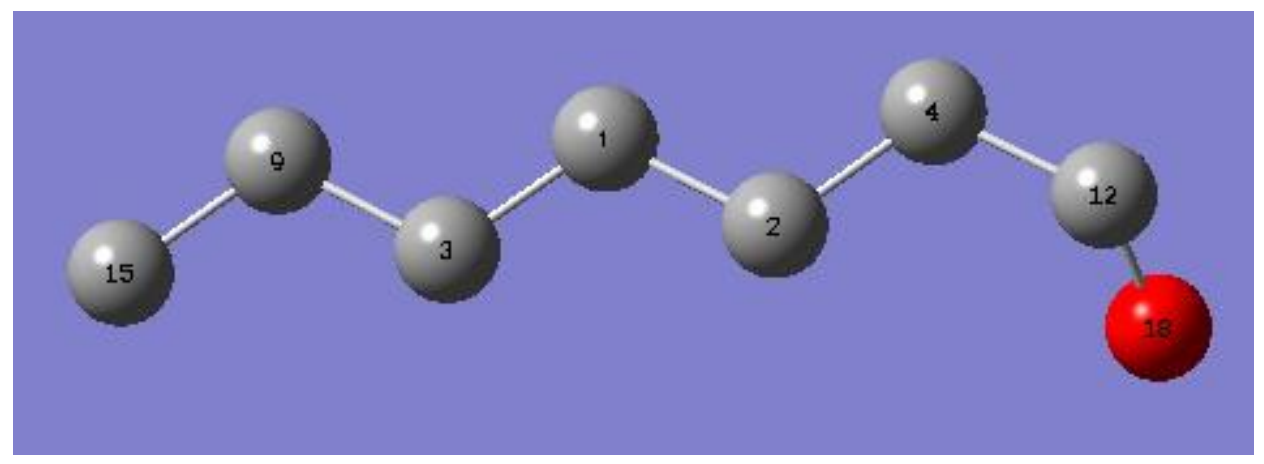




\section{Cyclohexanone (gas)}

$\mathrm{SCF}$ Done: $\mathrm{E}(\mathrm{UAPFD})=$

Zero-point correction=

Thermal correction to Energy=

Thermal correction to Enthalpy=

Thermal correction to Gibbs Free Energy=

Sum of electronic and zero-point Energies=

Sum of electronic and thermal Energies=

Sum of electronic and thermal Enthalpies=

Sum of electronic and thermal Free Energies=
$-309.731277644$

0.150804 (Hartree/Particle)

0.157334

0.158278

0.120377

$-309.580473$

$-309.573943$

$-309.572999$

$-309.610901$

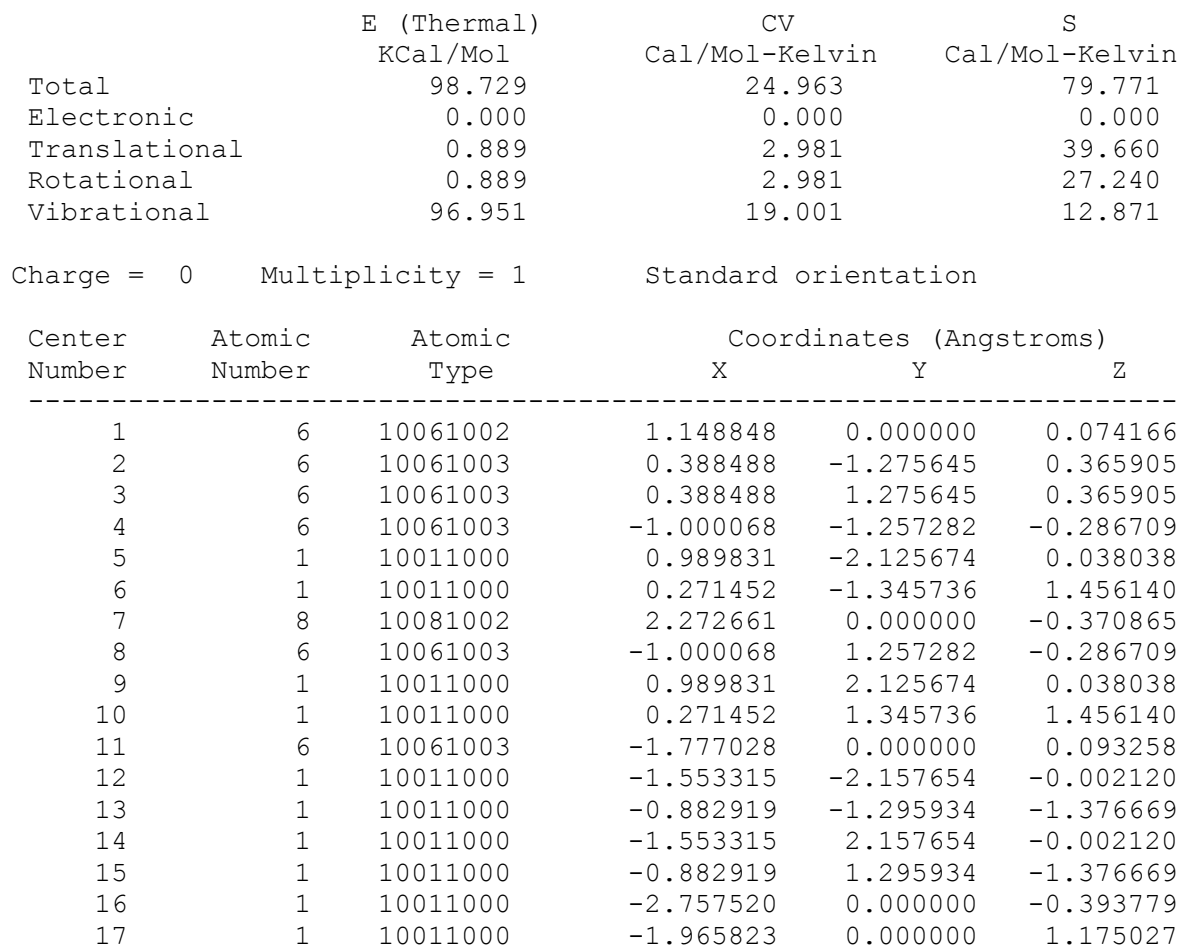

Summary of Natural Population Analysis:

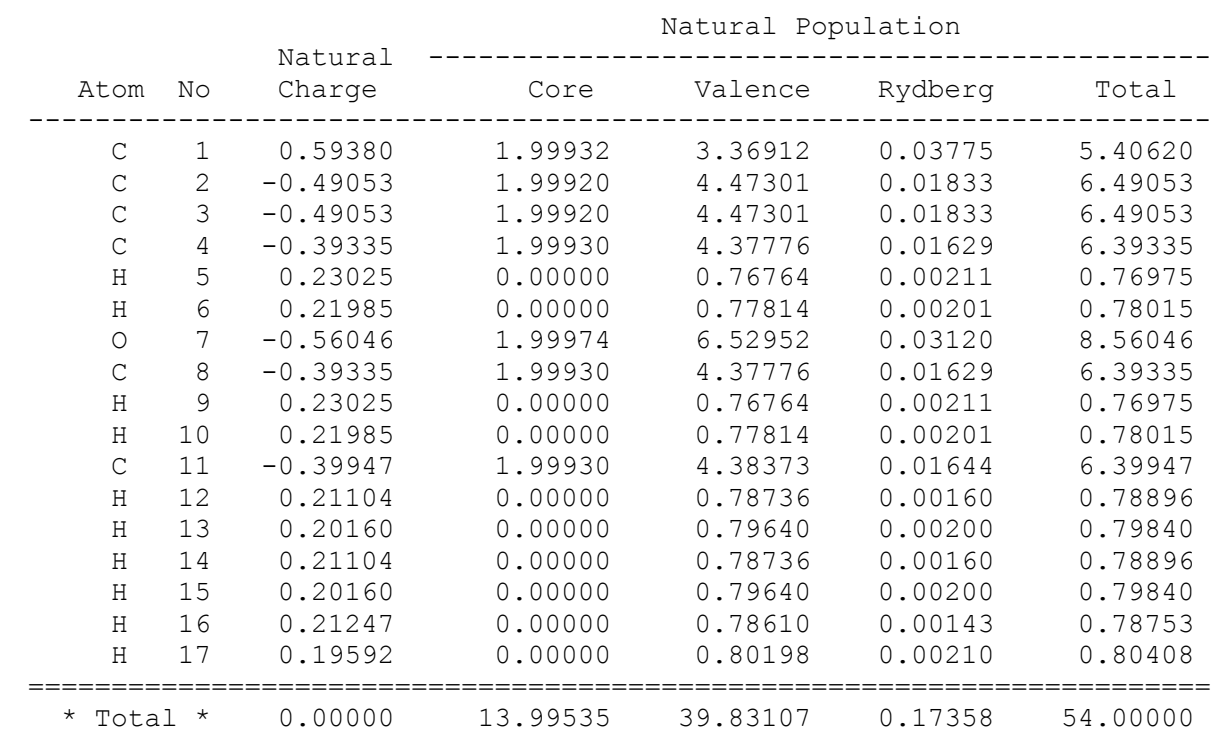

Maximum Force

0.000001

0.000015

YES 


$\begin{array}{lllll}\text { RMS } & \text { Force } & 0.000000 & 0.000010 & \text { YES } \\ \text { Maximum } & \text { Displacement } & 0.000011 & 0.000060 & \text { YES } \\ \text { RMS } & \text { Displacement } & 0.000002 & 0.000040 & \text { YES }\end{array}$

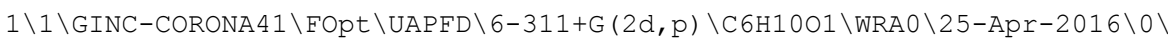
$\backslash \#$ opt $=($ calcfc, tight) freq uapfd $/ 6-311+g(2 d, p)$ pop=npa geom=connectivi ty int=(ultrafine, acc2e=12) \\1) Cyclohexanone chair clean Z - - opt=cal CfC uff fname=CyhexoneMM0322 2) CyhexoneMM0322.log --> (M5) \# opt=(calc $f_{C}$, tight) freq uapfd $/ 6-311+g(2 d, p)$ pop=npa geom=connectivity int=(ultr afine, acc2e=12) fname=Cyhexone0425.gjf $\backslash \backslash 0,1 \backslash C,-1.1521614327,-0.0000211$ $304,0.0668834492 \backslash C,-0.3925036031,1.2756360665,0.3603949662 \backslash C,-0.392467$ $7988,-1.2756544586,0.360406196 \backslash C, 0.997575969,1.2572890235,-0.288968218$ $9 \backslash \mathrm{H},-0.9930898697,2.1256549622,0.0311177051 \backslash \mathrm{H},-0.2780202906,1.34573345$ $57,1.4509006429 \backslash 0,-2.2749301954,-0.0000388021,-0.380776272 \backslash \mathrm{C}, 0.9976112$ $524,-1.2572741091,-0.2889571582 \backslash \mathrm{H},-0.9930302214,-2.1256931201,0.031136$ $3998 \backslash \mathrm{H},-0.2779825303,-1.3457390378,1.4509124924 \backslash \mathrm{C}, 1.7736622831,0.00002$ $00271,0.0928209678 \backslash \mathrm{H}, 1.5501428123,2.1576709043,-0.0030899521 \backslash \mathrm{H}, 0.88297$ $65183,1.2959352669,-1.3791996701 \backslash \mathrm{H}, 1.5502033669,-2.1576379672,-0.00307$ $09807 \backslash \mathrm{H}, 0.8830128789,-1.2959331534,-1.3791882662 \backslash \mathrm{H}, 2.7552909771,0.0000$ $316702,-0.3919206538 \backslash \mathrm{H}, 1.959925884,0.0000274023,1.1750293527 \backslash \backslash$ Version $=$

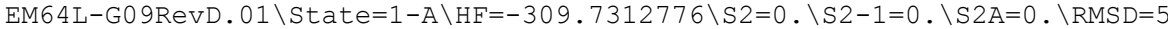
$.902 \mathrm{e}-09 \backslash \mathrm{RMSF}=6.274 \mathrm{e}-07 \backslash \mathrm{Dipole}=1.2798068,0.0000197,0.4026799 \backslash$ Quadrupol $\mathrm{e}=-5.0387505,2.8695015,2.169249,-0.0001182,-1.6757764,-0.0000266 \backslash \mathrm{PG}=\mathrm{C} 0$ $1[\mathrm{X}(\mathrm{C} 6 \mathrm{H} 10 \mathrm{O} 1)] \backslash \backslash @$

$\begin{array}{lllll}\text { Maximum } & \text { Force } & 0.000001 & 0.000015 & \text { YES } \\ \text { RMS } & \text { Force } & 0.000000 & 0.000010 & \text { YES } \\ \text { Maximum } & \text { Displacement } & 0.000011 & 0.000060 & \text { YES } \\ \text { RMS } & \text { Displacement } & 0.000002 & 0.000040 & \text { YES }\end{array}$

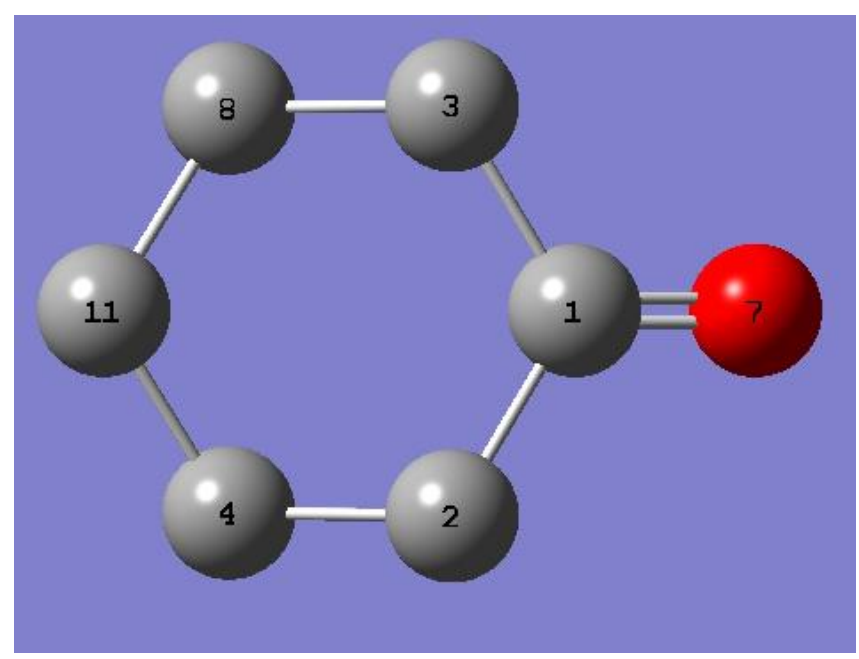




\section{Cyclohexanone (iefpcm)}

$\mathrm{SCF}$ Done: $\mathrm{E}(\mathrm{UAPFD})=$

Zero-point correction=

Thermal correction to Energy=

Thermal correction to Enthalpy=

Thermal correction to Gibbs Free Energy=

Sum of electronic and zero-point Energies=

Sum of electronic and thermal Energies=

Sum of electronic and thermal Enthalpies=

Sum of electronic and thermal Free Energies=
$-309.737020939$

0.150704 (Hartree/Particle)

0.157223

0.158167

0.120282

$-309.586317$

$-309.579798$

$-309.578853$

$-309.616739$

$\begin{array}{lccc} & \text { E (Thermal) } & \text { CV } & \text { S } \\ \text { KCal/Mol } & \text { Cal/Mol-Kelvin } & \text { Cal/Mol-Kelvin } \\ \text { Total } & 98.659 & 24.949 & 79.736 \\ \text { Electronic } & 0.000 & 0.000 & 0.000 \\ \text { Translational } & 0.889 & 2.981 & 39.660 \\ \text { Rotational } & 0.889 & 2.981 & 27.240 \\ \text { Vibrational } & 96.882 & 18.987 & 12.837\end{array}$

Charge $=0$ Multiplicity $=1 \quad$ Standard orientation

\begin{tabular}{|c|c|c|c|c|c|}
\hline \multirow{2}{*}{$\begin{array}{l}\text { Center } \\
\text { Number }\end{array}$} & \multirow{2}{*}{$\begin{array}{l}\text { Atomic } \\
\text { Number }\end{array}$} & \multirow{2}{*}{$\begin{array}{c}\text { Atomic } \\
\text { Type }\end{array}$} & \multicolumn{3}{|c|}{ Coordinates (Angstroms) } \\
\hline & & & $\mathrm{X}$ & $\mathrm{Y}$ & Z \\
\hline 1 & 6 & 10061002 & -0.416245 & -1.069800 & 0.000000 \\
\hline 2 & 6 & 10061003 & 0.162202 & -0.504738 & 1.273999 \\
\hline 3 & 6 & 10061003 & 0.162202 & -0.504738 & -1.273999 \\
\hline 4 & 6 & 10061003 & 0.162202 & 1.030389 & 1.256096 \\
\hline 5 & 1 & 10011000 & -0.388109 & -0.908069 & 2.126301 \\
\hline 6 & 1 & 10011000 & 1.199035 & -0.860891 & 1.342964 \\
\hline 7 & 8 & 10081002 & -1.290090 & -1.913744 & 0.000000 \\
\hline 8 & 6 & 10061003 & 0.162202 & 1.030389 & -1.256096 \\
\hline 9 & 1 & 10011000 & -0.388109 & -0.908069 & -2.126301 \\
\hline 10 & 1 & 10011000 & 1.199035 & -0.860891 & -1.342964 \\
\hline 11 & 6 & 10061003 & 0.837978 & 1.571041 & 0.000000 \\
\hline 12 & 1 & 10011000 & 0.656789 & 1.404219 & 2.157405 \\
\hline 13 & 1 & 10011000 & -0.873429 & 1.389027 & 1.295420 \\
\hline 14 & 1 & 10011000 & 0.656789 & 1.404219 & -2.157405 \\
\hline 15 & 1 & 10011000 & -0.873429 & 1.389027 & -1.295420 \\
\hline 16 & 1 & 10011000 & 0.812268 & 2.665285 & 0.000000 \\
\hline 17 & 1 & 10011000 & 1.896628 & 1.280832 & 0.000000 \\
\hline
\end{tabular}

Summary of Natural Population Analysis:

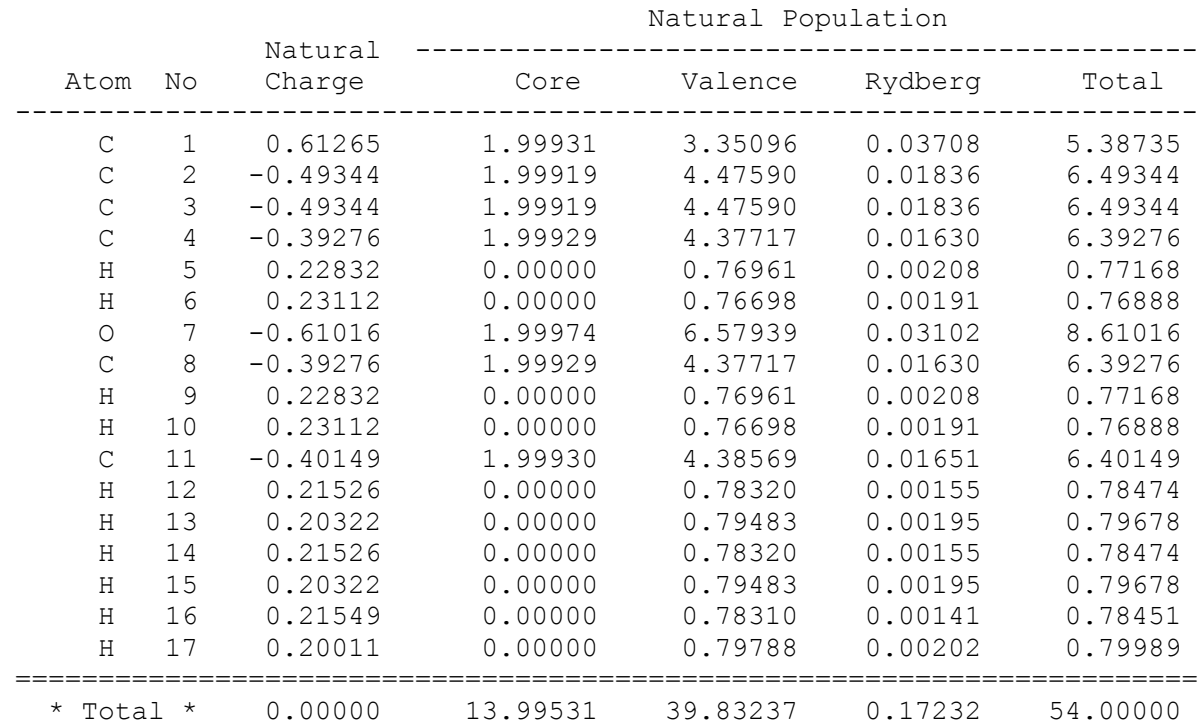




\begin{tabular}{|c|c|c|c|c|}
\hline Maximum & Force & 0.000002 & 0.000015 & YES \\
\hline RMS & Force & 0.000000 & 0.000010 & YES \\
\hline Maximum & Displacement & 0.000050 & 0.000060 & YES \\
\hline RMS & Displacement & 0.000006 & 0.000040 & YES \\
\hline
\end{tabular}

$1 \backslash 1 \backslash$ GINC-CORONA52 $\backslash$ FOpt \UAPFD $\backslash 6-311+\mathrm{G}(2 \mathrm{~d}, \mathrm{p}) \backslash \mathrm{C} 6 \mathrm{H} 1001 \backslash$ WRA $0 \backslash 26-\mathrm{Apr}-2016 \backslash 0 \backslash$ $\backslash \#$ opt $=($ calcfc, tight) freq uapfd/6-311+g $(2 \mathrm{~d}, \mathrm{p})$ scrf=(iefpcm, solvent=th f) pop=npa geom=connectivity int=(ultrafine, acc2e=12) \\1) Cyclohexanone chair clean $\mathrm{Z}-\rightarrow$ opt=(calcfc,tight) uff scrf=(iefpcm, solvent=thf) in $t=u l t r a f i n e ~ f n a m e=C y h e x o n e M M 0426 . g j f ~ 2)$ CyhexoneMM0426.log --> method_6

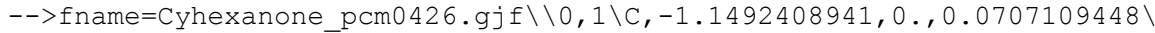
$C,-0.3929170802,1.27 \overline{3} 9993915,0.3568402083 \backslash C,-0.3929170802,-1.273999391$ $5,0.3568402083 \backslash \mathrm{C}, 0.998966209,1.2560957984,-0.2906760354 \backslash \mathrm{H},-0.990733407$ $3,2.1263006045,0.0280037582 \backslash \mathrm{H},-0.2785016074,1.3429642359,1.4471501446 \backslash$ $0,-2.2830234611,0 .,-0.3656191682 \backslash \mathrm{C}, 0.998966209,-1.2560957984,-0.290676$ $0354 \backslash \mathrm{H},-0.9907334073,-2.1263006045,0.0280037582 \backslash \mathrm{H},-0.2785016074,-1.342$ $9642359,1.4471501446 \backslash \mathrm{C}, 1.7742109257,0 ., 0.0939953645 \backslash \mathrm{H}, 1.5465297364,2.1$ $574052529,0.0000792649 \backslash \mathrm{H}, 0.8873102212,1.2954203271,-1.3809454862 \backslash \mathrm{H}, 1.5$ $465297364,-2.1574052529,0.0000792649 \backslash \mathrm{H}, 0.8873102212,-1.2954203271,-1.3$ $809454862 \backslash \mathrm{H}, 2.7555059737,0 .,-0.3908675437 \backslash \mathrm{H}, 1.957620312,0 ., 1.176271694$ $\backslash \backslash$ Version=EM64L-G09RevD.01 \State $=1-A^{\prime} \backslash H F=-309.7370209 \backslash S 2=0 . \backslash S 2-1=0 . \backslash S 2$ $\mathrm{A}=0 . \backslash \mathrm{RMSD}=7.139 \mathrm{e}-09 \backslash \mathrm{RMSF}=2.263 \mathrm{e}-06 \backslash \mathrm{Dipole}=1.6041975,0 ., 0.513494 \backslash$ Quadru pole $=-5.9278967,3.3453859,2.5825108,0 .,-1.8611928,0 . \backslash P G=C S \quad[S G(C 2 H 2 O 1)$ , $\mathrm{X}(\mathrm{C} 4 \mathrm{H} 8)] \backslash \backslash \mathrm{e}$

$\begin{array}{lllll}\text { Maximum } & \text { Force } & 0.000002 & 0.000015 & \text { YES } \\ \text { RMS } & \text { Force } & 0.000000 & 0.000010 & \text { YES } \\ \text { Maximum } & \text { Displacement } & 0.000028 & 0.000060 & \text { YES } \\ \text { RMS } & \text { Displacement } & 0.000004 & 0.000040 & \text { YES }\end{array}$

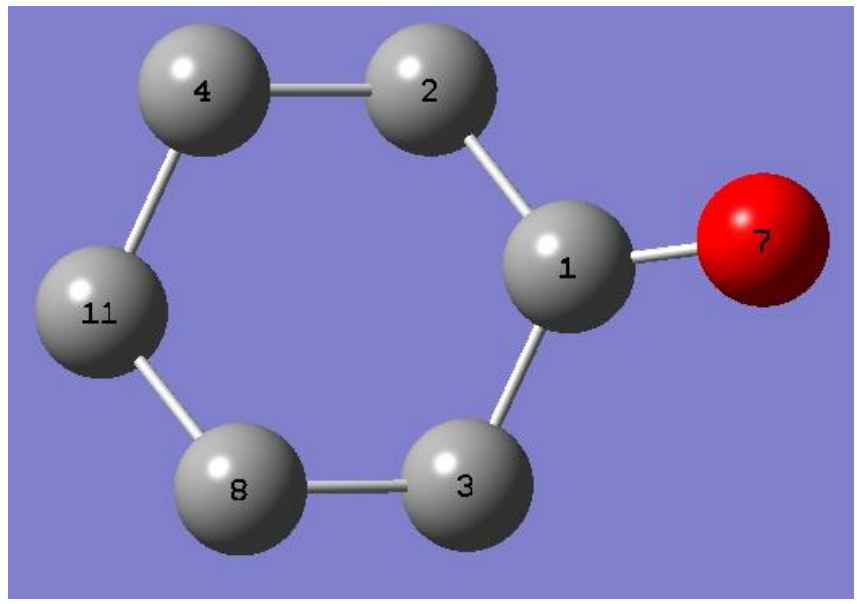




\section{Cyclohexanone radical ion (gas)}

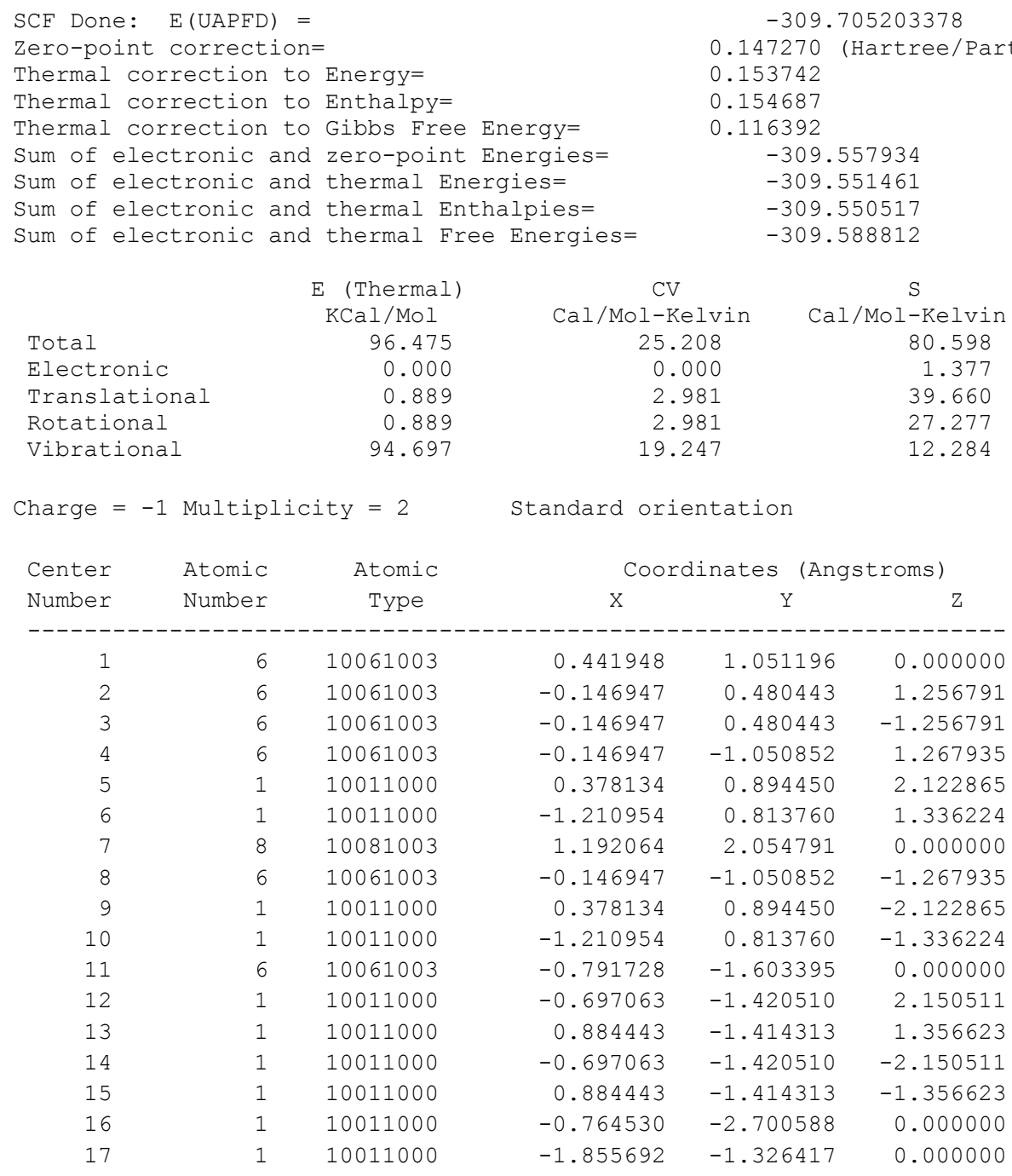

Summary of Natural Population Analysis:

Natural Population

\begin{tabular}{|c|c|c|c|c|c|c|}
\hline Atom & No & $\begin{array}{l}\text { Natural } \\
\text { Charge }\end{array}$ & Core & Valence & Rydberg & Total \\
\hline C & 1 & 0.41364 & 1.99922 & 3.54248 & 0.04465 & 5.58636 \\
\hline C & 2 & -0.54113 & 1.99918 & 4.46134 & 0.08061 & 6.54113 \\
\hline C & 3 & -0.54113 & 1.99918 & 4.46134 & 0.08061 & 6.54113 \\
\hline C & 4 & -0.42849 & 1.99927 & 4.37555 & 0.05367 & 6.42849 \\
\hline $\mathrm{H}$ & 5 & 0.20469 & 0.00000 & 0.79096 & 0.00435 & 0.79531 \\
\hline $\mathrm{H}$ & 6 & 0.16119 & 0.00000 & 0.82231 & 0.01651 & 0.83881 \\
\hline O & 7 & -0.71127 & 1.99977 & 6.68805 & 0.02345 & 8.71127 \\
\hline C & 8 & -0.42849 & 1.99927 & 4.37555 & 0.05367 & 6.42849 \\
\hline $\mathrm{H}$ & 9 & 0.20469 & 0.00000 & 0.79096 & 0.00435 & 0.79531 \\
\hline $\mathrm{H}$ & 10 & 0.16119 & 0.00000 & 0.82231 & 0.01651 & 0.83881 \\
\hline C & 11 & -0.55589 & 1.99924 & 4.39116 & 0.16549 & 6.55589 \\
\hline $\mathrm{H}$ & 12 & 0.17082 & 0.00000 & 0.82204 & 0.00714 & 0.82918 \\
\hline $\mathrm{H}$ & 13 & 0.18052 & 0.00000 & 0.81580 & 0.00369 & 0.81948 \\
\hline
\end{tabular}




$\begin{array}{ccccccc}\mathrm{H} & 14 & 0.17082 & 0.00000 & 0.82204 & 0.00714 & 0.82918 \\ \mathrm{H} & 15 & 0.18052 & 0.00000 & 0.81580 & 0.00369 & 0.81948 \\ \mathrm{H} & 16 & 0.18076 & 0.00000 & 0.81189 & 0.00735 & 0.81924 \\ \mathrm{H} & 17 & 0.17759 & 0.00000 & 0.80824 & 0.01417 & 0.82241 \\ ================================================================ \\ \text { * Total * } & -1.00000 & 13.99513 & 40.41782 & 0.58705 & 55.00000\end{array}$

$\begin{array}{lllll}\text { Maximum } & \text { Force } & 0.000000 & 0.000015 & \text { YES } \\ \text { RMS } & \text { Force } & 0.000000 & 0.000010 & \text { YES } \\ \text { Maximum } & \text { Displacement } & 0.000006 & 0.000060 & \text { YES } \\ \text { RMS } & \text { Displacement } & 0.000001 & 0.000040 & \text { YES }\end{array}$

$1 \backslash 1 \backslash G I N C-M A I A \backslash F O p t \backslash U A P F D \backslash 6-311+G(2 d, p) \backslash C 6 H 1001(1-, 2) \backslash$ WRA0 $\backslash 25-A p r-2016 \backslash$ $0 \backslash \backslash \#$ opt $=($ calcfc, tight) freq uapfd $/ 6-311+g(2 d, p)$ pop=npa geom=connecti vity int=(ultrafine, acc $2 e=12) \backslash \backslash 1)$ Cyclohexanone radical anion chair $\mathrm{z} c$ lean - $>$ opt=calcfc uff fname=Cyclohexol_radanMM0322 2) Cyclohexol_rada nMM0322. log -->(M5) opt=(calcfc,tight) freq uapfd $/ 6-311+g(2 d, p)$ pop=np a geom=connectivity int=(ultrafine, acc2e=12) $-\rightarrow$ fname=Cyclohexol_rada n0425.gjf \\-1,2\C,0.4419745671,1.0466133004,0.\C, $-0.121055455,0.450329$ $9301,1.2567912293 \backslash \mathrm{C},-0.121055455,0.4503299301,-1.2567912293 \backslash \mathrm{C},-0.05321$ $27793,-1.0794606816,1.2679348053 \backslash \mathrm{H}, 0.3851674753,0.887194051,2.12286518$ $71 \backslash \mathrm{H},-1.1987851561,0.7361799666,1.3362242512 \backslash 0,1.1468903528,2.08245642$ $75,0 . \backslash \mathrm{C},-0.0532127793,-1.0794606816,-1.2679348053 \backslash \mathrm{H}, 0.3851674753,0.887$ $194051,-2.1228651871 \backslash \mathrm{H},-1.1987851561,0.7361799666,-1.3362242512 \backslash \mathrm{C},-0.6$ $728804462,-1.6600277735,0 . \backslash \mathrm{H},-0.5864112006,-1.4731290223,2.1505113124 \backslash$ $\mathrm{H}, 0.9932674464,-1.3968703477,1.3566225419 \backslash \mathrm{H},-0.5864112006,-1.473129022$ $3,-2.1505113124 \backslash \mathrm{H}, 0.9932674464,-1.3968703477,-1.3566225419 \backslash \mathrm{H},-0.597099$ $4445,-2.7549382917,0 . \backslash \mathrm{H},-1.7480716944,-1.4304604537,0 . \backslash \backslash$ Version=EM64LG09RevD.01 \State $=2-A^{\prime} \backslash \mathrm{HF}=-309.7052034 \backslash \mathrm{S} 2=0.752082 \backslash \mathrm{S} 2-1=0 . \backslash \mathrm{S} 2 \mathrm{~A}=0.750003$ $\backslash \mathrm{RMSD}=4.721 \mathrm{e}-09 \backslash \mathrm{RMSF}=2.912 \mathrm{e}-07 \backslash \mathrm{Dipole}=0.6539882,-1.1856969,0 . \backslash$ Quadrupo $l e=-0.2265484,-3.3411806,3.567729,-6.6384913,0 ., 0 . \backslash P G=C S \quad[S G(C 2 H 2 O 1), x$ $(\mathrm{C} 4 \mathrm{H} 8)] \backslash \backslash \mathrm{Q}$

$\begin{array}{lllll}\text { Maximum } & \text { Force } & 0.000000 & 0.000015 & \text { YES } \\ \text { RMS } & \text { Force } & 0.000000 & 0.000010 & \text { YES } \\ \text { Maximum } & \text { Displacement } & 0.000006 & 0.000060 & \text { YES } \\ \text { RMS } & \text { Displacement } & 0.000002 & 0.000040 & \text { YES }\end{array}$

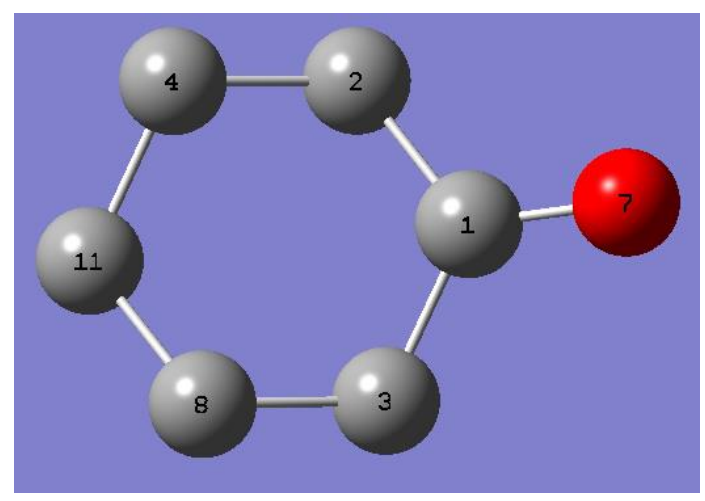




\section{Cyclohexanone radical ion (iefpcm)}

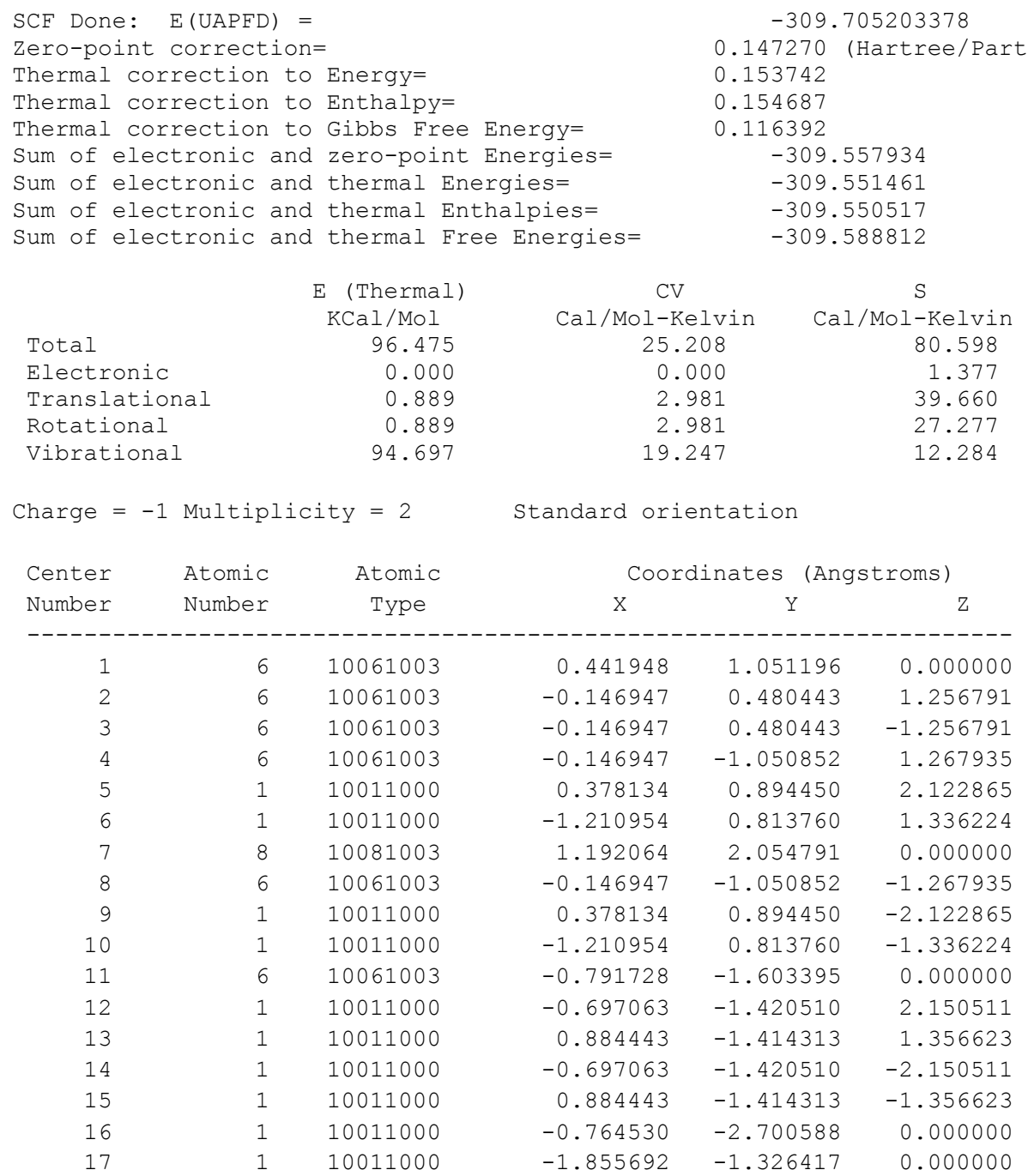

Summary of Natural Population Analysis:

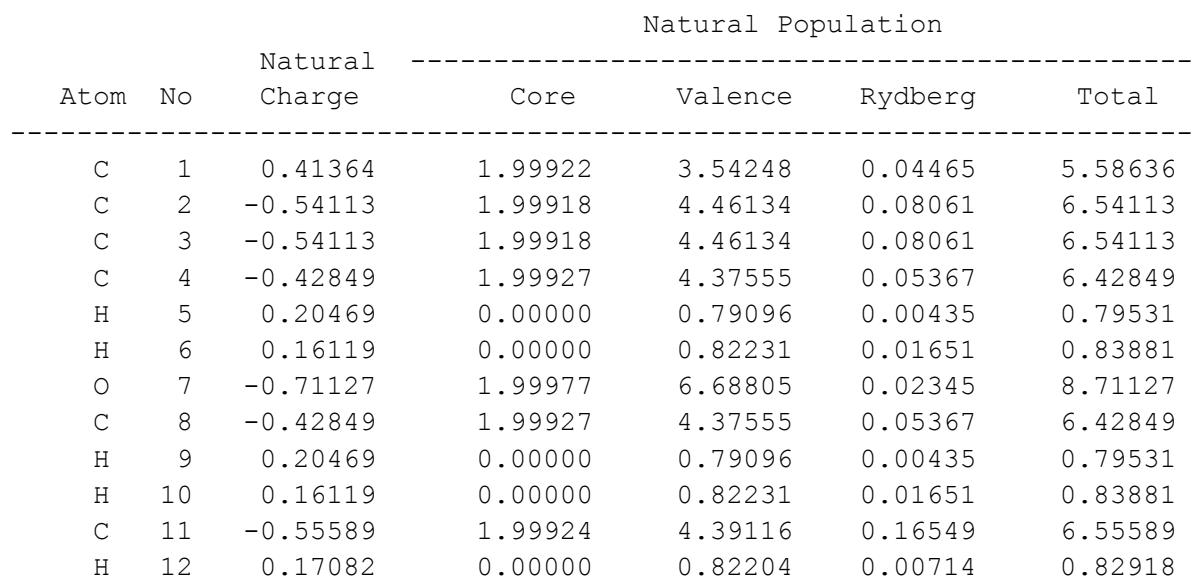




$\begin{array}{ccccccc}\mathrm{H} & 13 & 0.18052 & 0.00000 & 0.81580 & 0.00369 & 0.81948 \\ \mathrm{H} & 14 & 0.17082 & 0.00000 & 0.82204 & 0.00714 & 0.82918 \\ \mathrm{H} & 15 & 0.18052 & 0.00000 & 0.81580 & 0.00369 & 0.81948 \\ \mathrm{H} & 16 & 0.18076 & 0.00000 & 0.81189 & 0.00735 & 0.81924 \\ \mathrm{H} & 17 & 0.17759 & 0.00000 & 0.80824 & 0.01417 & 0.82241 \\ =========================================================== \\ \text { * Total * } & -1.00000 & 13.99513 & 40.41782 & 0.58705 & 55.00000\end{array}$

$\begin{array}{lllll}\text { Maximum } & \text { Force } & 0.000000 & 0.000015 & \text { YES } \\ \text { RMS } & \text { Force } & 0.000000 & 0.000010 & \text { YES } \\ \text { Maximum } & \text { Displacement } & 0.000006 & 0.000060 & \text { YES } \\ \text { RMS } & \text { Displacement } & 0.000001 & 0.000040 & \text { YES }\end{array}$

$1 \backslash 1 \backslash G I N C-M A I A \backslash F O p t \backslash U A P F D \backslash 6-311+G(2 d, p) \backslash C 6 H 1001(1-, 2) \backslash$ WRA $\backslash 25-A p r-2016 \backslash$ $0 \backslash \backslash \#$ opt=(calcfc,tight) freq uapfd/6-311+g $(2 d, p)$ pop=npa geom=connecti vity int=(ultrafine, acc $2 e=12) \backslash \backslash 1$ ) Cyclohexanone radical anion chair $\mathrm{Z} \mathrm{c}$ lean --> opt=calcfc uff fname=Cyclohexol_radanMM0322 2) Cyclohexol_rada nMM0322. log $-->$ (M5) opt=(calcfc,tight) freq uapfd/6-311+g (2d,p) pop=np a geom=connectivity int=(ultrafine, acc2e=12) $-->$ fname=Cyclohexol_rada n0425.gjf $\backslash \backslash-1,2 \backslash C, 0.4419745671,1.0466133004,0 . \backslash C,-0.121055455,0.4 \overline{5} 0329$ $9301,1.2567912293 \backslash C,-0.121055455,0.4503299301,-1.2567912293 \backslash C,-0.05321$ $27793,-1.0794606816,1.2679348053 \backslash \mathrm{H}, 0.3851674753,0.887194051,2.12286518$ $71 \backslash \mathrm{H},-1.1987851561,0.7361799666,1.3362242512 \backslash 0,1.1468903528,2.08245642$ $75,0 . \backslash \mathrm{C},-0.0532127793,-1.0794606816,-1.2679348053 \backslash \mathrm{H}, 0.3851674753,0.887$ $194051,-2.1228651871 \backslash \mathrm{H},-1.1987851561,0.7361799666,-1.3362242512 \backslash \mathrm{C},-0.6$ $728804462,-1.6600277735,0 . \backslash \mathrm{H},-0.5864112006,-1.4731290223,2.1505113124 \backslash$ $\mathrm{H}, 0.9932674464,-1.3968703477,1.3566225419 \backslash \mathrm{H},-0.5864112006,-1.473129022$ $3,-2.1505113124 \backslash \mathrm{H}, 0.9932674464,-1.3968703477,-1.3566225419 \backslash \mathrm{H},-0.597099$ $4445,-2.7549382917,0 . \backslash \mathrm{H},-1.7480716944,-1.4304604537,0 . \backslash \backslash$ Version=EM64LG09RevD.01 \State=2-A $\backslash \mathrm{HF}=-309.7052034 \backslash \mathrm{S} 2=0.752082 \backslash \mathrm{S} 2-1=0 . \backslash \mathrm{S} 2 \mathrm{~A}=0.750003$ $\backslash \mathrm{RMSD}=4.721 \mathrm{e}-09 \backslash \mathrm{RMSF}=2.912 \mathrm{e}-07 \backslash \mathrm{Dipole}=0.6539882,-1.1856969,0 . \backslash$ Quadrupo $l e=-0.2265484,-3.3411806,3.567729,-6.6384913,0 ., 0 . \backslash P G=C S \quad[S G(C 2 H 2 O 1), X$ $(\mathrm{C} 4 \mathrm{H} 8)] \backslash \backslash @$

\begin{tabular}{|c|c|c|c|c|}
\hline Maximum & Force & 0.000000 & 0.000015 & YES \\
\hline RMS & Force & 0.000000 & 0.000010 & YES \\
\hline Maximum & Displacement & 0.000006 & 0.000060 & YES \\
\hline DNo & Displacement & 0 & 0000040 & YFS \\
\hline
\end{tabular}

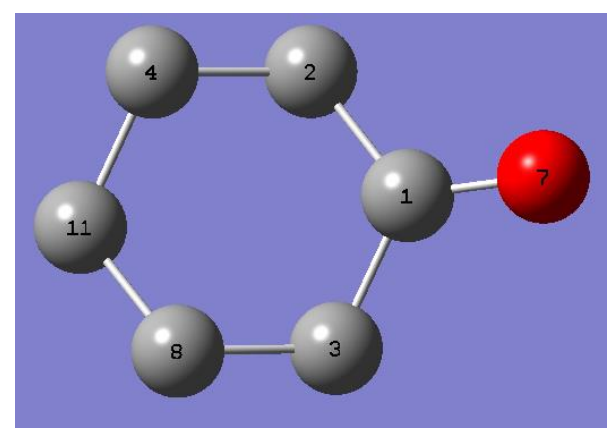




\section{d-Valerolactone (gas)}

SCF Done: E (UAPFD) =

Zero-point correction=

Thermal correction to Energy=

Thermal correction to Enthalpy=

Thermal correction to Gibbs Free Energy=

Sum of electronic and zero-point Energies=

Sum of electronic and thermal Energies=

Sum of electronic and thermal Enthalpies=

Sum of electronic and thermal Free Energies=
$-345.634544204$

0.127597 (Hartree/Particle)

0.133837

0.134781

0.097271

$-345.506947$

$-345.500707$

$-345.499763$

$-345.537273$

$\mathrm{CV}$

$\begin{array}{lccc} & \text { KCal/Mol } & \text { Cal/Mol-Kelvin } & \text { Cal/Mol-Kelvin } \\ \text { Total } & 83.984 & 23.066 & 78.946 \\ \text { Electronic } & 0.000 & 0.000 & 0.000 \\ \text { Translational } & 0.889 & 2.981 & 39.720 \\ \text { Rotational } & 0.889 & 2.981 & 27.108 \\ \text { Vibrational } & 82.207 & 17.105 & 12.119\end{array}$

Charge $=0$ Multiplicity $=1 \quad$ Standard orientation

\begin{tabular}{|c|c|c|c|c|c|}
\hline \multirow{2}{*}{$\begin{array}{l}\text { Center } \\
\text { Number }\end{array}$} & \multirow{2}{*}{$\begin{array}{l}\text { Atomic } \\
\text { Number }\end{array}$} & \multirow{2}{*}{$\begin{array}{c}\text { Atomic } \\
\text { Type }\end{array}$} & \multicolumn{3}{|c|}{ Coordinates (Angstroms) } \\
\hline & & & $\mathrm{x}$ & $Y$ & Z \\
\hline 1 & 6 & 10061000 & 1.129341 & -0.016775 & -0.013919 \\
\hline 2 & 6 & 10061003 & -0.958053 & -1.268049 & 0.170185 \\
\hline 3 & 6 & 10061003 & -1.723319 & -0.059837 & -0.325012 \\
\hline 4 & 6 & 10061003 & -1.119226 & 1.189140 & 0.295267 \\
\hline 5 & 6 & 10061003 & 0.345381 & 1.277357 & -0.113595 \\
\hline 6 & 1 & 10011000 & -1.262243 & -2.183463 & -0.339501 \\
\hline 7 & 1 & 10011000 & -1.671041 & -0.004063 & -1.418601 \\
\hline 8 & 1 & 10011000 & -2.777146 & -0.180309 & -0.055782 \\
\hline 9 & $\begin{array}{l}1 \\
1\end{array}$ & 10011000 & -1.653483 & 2.092131 & -0.013041 \\
\hline 10 & 1 & 10011000 & -1.202956 & 1.131348 & 1.387067 \\
\hline 11 & 1 & 10011000 & 0.421630 & 1.571760 & -1.167841 \\
\hline 12 & 1 & 10011000 & 0.901538 & 2.026039 & 0.452714 \\
\hline 13 & 1 & 10011000 & -1.123684 & -1.408503 & 1.244580 \\
\hline 14 & 8 & 10081000 & 2.328887 & -0.030790 & 0.012260 \\
\hline 15 & 8 & 10081000 & 0.461443 & -1.191204 & -0.033153 \\
\hline
\end{tabular}

Summary of Natural Population Analysis:

\begin{tabular}{|c|c|c|c|c|c|c|}
\hline Atom & No & $\begin{array}{l}\text { Natural } \\
\text { Charge }\end{array}$ & Core & Valence & Rydberg & $\begin{array}{l}\text {-------- } \\
\text { Total }\end{array}$ \\
\hline $\mathrm{C}$ & 1 & 0.82478 & 1.99935 & 3.13805 & 0.03782 & 5.17522 \\
\hline $\mathrm{C}$ & 2 & -0.03343 & 1.99919 & 4.01366 & 0.02058 & 6.03343 \\
\hline C & 3 & -0.43034 & 1.99930 & 4.41581 & 0.01523 & 6.43034 \\
\hline $\mathrm{C}$ & 4 & -0.40257 & 1.99930 & 4.38649 & 0.01678 & 6.40257 \\
\hline $\mathrm{C}$ & 5 & -0.50795 & 1.99921 & 4.49060 & 0.01813 & 6.50795 \\
\hline $\mathrm{H}$ & 6 & 0.19642 & 0.00000 & 0.80218 & 0.00140 & 0.80358 \\
\hline $\mathrm{H}$ & 7 & 0.20669 & 0.00000 & 0.79120 & 0.00211 & 0.79331 \\
\hline $\mathrm{H}$ & 8 & 0.22157 & 0.00000 & 0.77689 & 0.00154 & 0.77843 \\
\hline $\mathrm{H}$ & 9 & 0.21392 & 0.00000 & 0.78442 & 0.00166 & 0.78608 \\
\hline $\mathrm{H}$ & 10 & 0.20243 & 0.00000 & 0.79556 & 0.00201 & 0.79757 \\
\hline $\mathrm{H}$ & 11 & 0.23459 & 0.00000 & 0.76368 & 0.00173 & 0.76541 \\
\hline $\mathrm{H}$ & 12 & 0.24127 & 0.00000 & 0.75712 & 0.00160 & 0.75873 \\
\hline $\mathrm{H}$ & 13 & 0.17824 & 0.00000 & 0.81978 & 0.00198 & 0.82176 \\
\hline 0 & 14 & -0.58217 & 1.99973 & 6.55179 & 0.03065 & 8.58217 \\
\hline 0 & 15 & -0.56346 & 1.99970 & 6.53374 & 0.03002 & 8.56346 \\
\hline \multirow{2}{*}{\multicolumn{7}{|c|}{$\begin{array}{c}========== \\
\text { * Total * }\end{array}$}} \\
\hline \multirow{2}{*}{\multicolumn{2}{|c|}{$\begin{array}{c}\text { * Total * } \\
\text { Iaximum Force }\end{array}$}} & 0.00000 & 13.99578 & 39.82098 & 0.18325 & 54.00000 \\
\hline & & & 0.000001 & 0.000015 & YES & \\
\hline RMS & Force & & 0.000000 & 0.000010 & YES & \\
\hline Maximum & Displa & aceme & 0.000043 & 0.000060 & YES & \\
\hline RMS & Displa & acement & 0.000008 & 0.000040 & YES & \\
\hline
\end{tabular}


$1 \backslash 1 \backslash$ GINC-CORONA4 9 \FOpt \UAPFD $\backslash 6-311+\mathrm{G}(2 \mathrm{~d}, \mathrm{p}) \backslash \mathrm{C} 5$ H8O2 \WRA0 $\backslash 26-A p r-2016 \backslash 0 \backslash \backslash$ \# opt=(calcfc,tight) freq uapfd/6-311+g $(2 d, p)$ pop=npa geom=connectivit y int=(ultrafine, acc $2 e=12) \backslash \backslash 1)$ Build dVL chair - clean $->$ opt=(calcfc, tight) uff int=ultrafine fname=dVL MM0426.gjf 2) dVL MM0426.log --> (M5 ) \# opt $=($ calcfc, tight) freq uapfd $/ 6-311+g(2 d, p)$ pop=npa geom=connectivi ty int $=($ ultrafine, acc2e=12) fname $=d V L=0426 . g j f \backslash \backslash 0,1 \backslash C, 1.1264290975,-0$. $0377563964,-0.0131356023 \backslash \mathrm{C},-0.9888636 \overline{4} 42,-1.226198063,0.2513251673 \backslash C_{,}-$ $1.7308456885,-0.0194110685,-0.2813614126 \backslash \mathrm{C},-1.0859191427,1.2373783303$, $0.2791465269 \backslash \mathrm{C}, 0.3740030576,1.2711956032,-0.1538747598 \backslash \mathrm{H},-1.3238870028$ $,-2.153131832,-0.216486551 \backslash \mathrm{H},-1.6939634381,-0.0089001138,-1.3769479097$ $\backslash \mathrm{H},-2.7831256996,-0.1016768966,0.0075826006 \backslash \mathrm{H},-1.6018436664,2.14080589$ $02,-0.0577763172 \backslash \mathrm{H},-1.1543184121,1.2255938809,1.3734776548 \backslash \mathrm{H}, 0.4414823$ $134,1.5210199858,-1.2201563752 \backslash \mathrm{H}, 0.9575367408,2.0273356336,0.373647784$ $5 \backslash \mathrm{H},-1.1414841404,-1.3191279957,1.3327848973 \backslash 0,2.3254975244,-0.0817517$ $231,-0.0035857204 \backslash 0,0.4288341011,-1.194332235,0.0247430168 \backslash \backslash$ Version $=E M$ 64L-G0 9RevD.01 \State $=1-\mathrm{A} \backslash \mathrm{HF}=-345.6345442 \backslash \mathrm{S} 2=0 . \backslash \mathrm{S} 2-1=0 . \backslash \mathrm{S} 2 \mathrm{~A}=0 . \backslash \mathrm{RMSD}=8.6$ $97 e-09 \backslash \mathrm{RMSF}=1.469 \mathrm{e}-06 \backslash \mathrm{Dipole}=-1.8758463,0.5263768,0.0249705 \backslash$ Quadrupole $=-4.575869,2.0749736,2.5008955,2.2539127,-0.0952855,-0.286784 \backslash P G=C 01 \quad[$ $\mathrm{X}(\mathrm{C} 5 \mathrm{H} 8 \mathrm{O} 2)] \backslash \backslash \mathrm{e}$

$\begin{array}{lllll}\text { Maximum } & \text { Force } & 0.000001 & 0.000015 & \text { YES } \\ \text { RMS } & \text { Force } & 0.000000 & 0.000010 & \text { YES } \\ \text { Maximum } & \text { Displacement } & 0.000069 & 0.000060 & \text { NO } \\ \text { RMS } & \text { Displacement } & 0.000012 & 0.000040 & \text { YES }\end{array}$

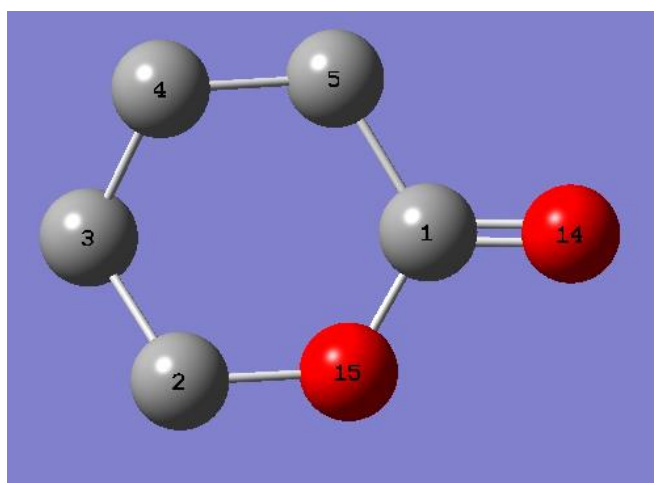




\section{d-Valerolactone (iefpcm)}

SCF Done: $\mathrm{E}(\mathrm{UAPFD})=$

Zero-point correction=

Thermal correction to Energy=

Thermal correction to Enthalpy=

Thermal correction to Gibbs Free Energy=

Sum of electronic and zero-point Energies=

Sum of electronic and thermal Energies=

Sum of electronic and thermal Enthalpies=

Sum of electronic and thermal Free Energies=

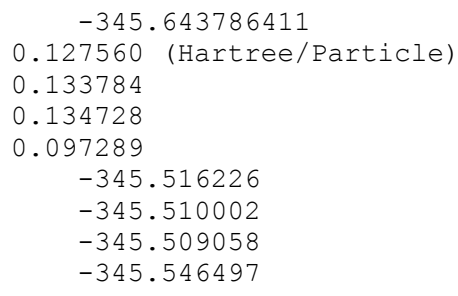

$\mathrm{CV}$

$\begin{array}{rr}\text { Cal/Mol-Kelvin } & \text { Cal/Mol-Kelvin } \\ 23.069 & 78.797 \\ 0.000 & 0.000 \\ 2.981 & 39.720 \\ 2.981 & 27.107 \\ 17.108 & 11.970\end{array}$

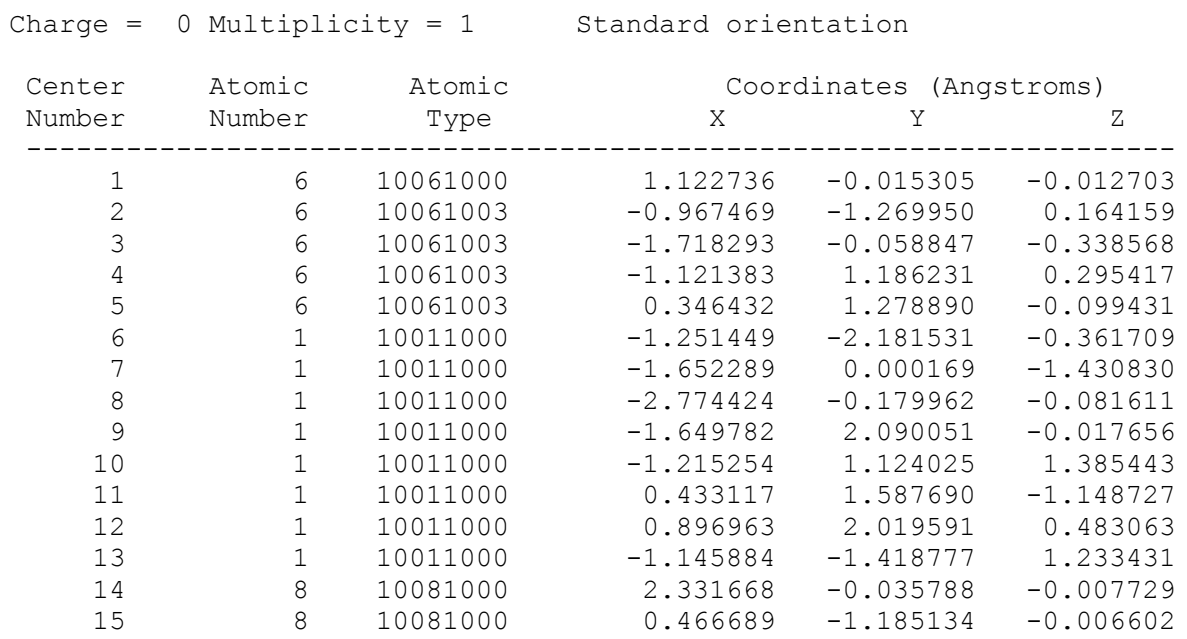

Summary of Natural Population Analysis:

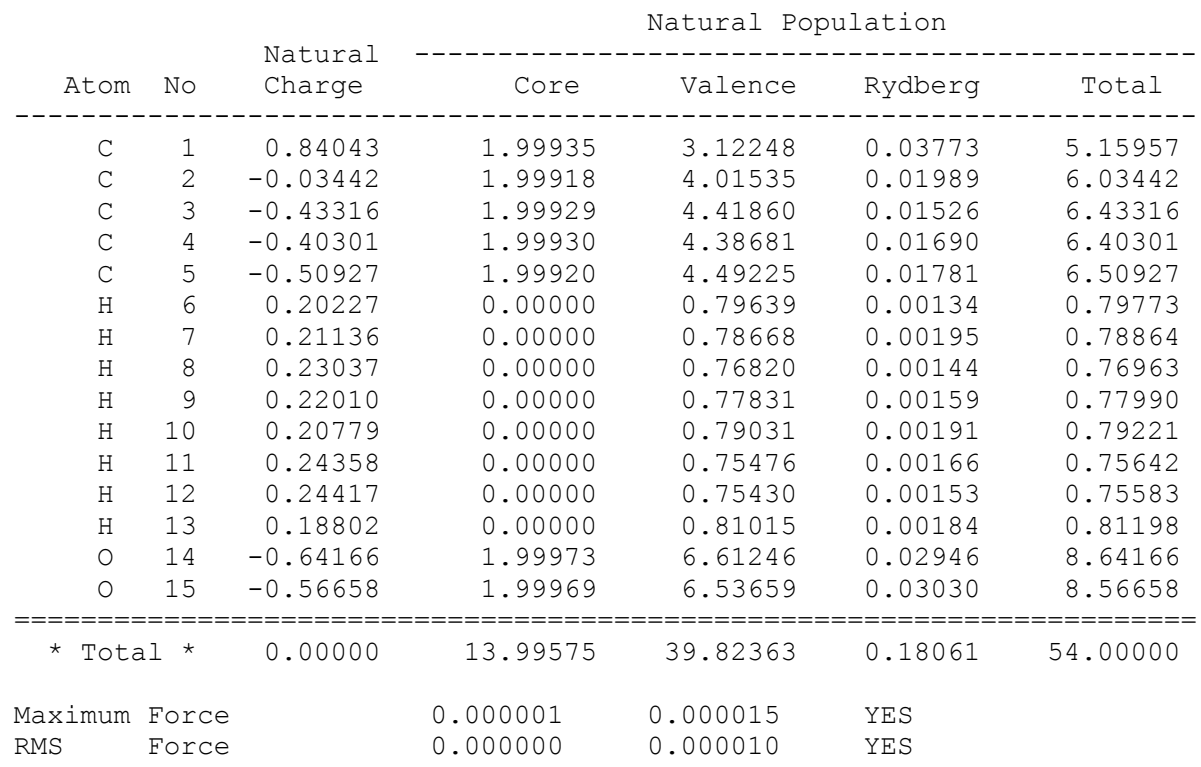




$\begin{array}{lllll}\text { Maximum } & \text { Displacement } & 0.000036 & 0.000060 & \text { YES } \\ \text { RMS } & \text { Displacement } & 0.000006 & 0.000040 & \text { YES }\end{array}$

$1 \backslash 1 \backslash$ GINC-CORONA31 \FOpt \UAPFD $\backslash 6-311+\mathrm{G}(2 \mathrm{~d}, \mathrm{p}) \backslash \mathrm{C} 5 \mathrm{H} 802 \backslash$ WRA $0 \backslash 27-A p r-2016 \backslash 0 \backslash \backslash$ \# opt $=($ calcfc,tight $)$ freq uapfd/6-311+g $(2 d, p)$ scrf=(iefpcm, solvent=thf ) $\mathrm{pop}=\mathrm{npa}$ geom=connectivity int=(ultrafine, acc2e=12) \\1)Build dVL chai $r$ - clean $->$ opt=(calcfc,tight) uff int=ultrafine - $>$ fname=dVL_MM0426 .gjf 2) dVL MM0426.gjf $-\rightarrow$ opt=(calcfc,tight) uff scrf=(iefpcm, solvent= thf) int=ulttrafine - $>$ fname=dVL_MMpcm0427.gjf 3) dVL_MMpcm0427.log --> (M6) \# opt=(calcfc, tight) freq uapfd/6-311+g(2d,p) scrf=(iefpcm, solvent =thf) pop=npa geom=connectivity int=(ultrafine, acc2e=12) fname=dVL_pcm $0427 . g j f \backslash \backslash 0,1 \backslash C, 1.1193079337,-0.0363100299,-0.0207298813 \backslash C,-0.99729334$ $12,-1.2264349745,0.2585095688 \backslash \mathrm{C},-1.7285580313,-0.020217031,-0.28327585$ $69 \backslash \mathrm{C},-1.0863044188,1.2363620925,0.2797313343 \backslash \mathrm{C}, 0.3740747133,1.27248369$ $35,-0.1503360593 \backslash \mathrm{H},-1.315954128,-2.1532531025,-0.2185924214 \backslash \mathrm{H},-1.68580$ $21504,-0.0127173168,-1.3782601592 \backslash \mathrm{H},-2.7813248079,-0.1018123585,0.0015$ $655644 \backslash \mathrm{H},-1.5987209861,2.1385265517,-0.0632547116 \backslash \mathrm{H},-1.1570419353,1.22$ $63049496,1.3732270842 \backslash \mathrm{H}, 0.4447614899,1.5308363604,-1.2143619341 \backslash \mathrm{H}, 0.95$ $61117332,2.0242526398,0.3854790118 \backslash \mathrm{H},-1.1551772419,-1.3217237728,1.337$ $081568 \backslash 0,2.3271442484,-0.0882001262,-0.0407300559 \backslash 0,0.4343179223,-1.18$ $70665753,0.0533229483 \backslash \backslash$ Version=EM64L-G09RevD.01 $\backslash$ State $=1-A \backslash H F=-345.6437$ $864 \backslash \mathrm{S} 2=0 . \backslash \mathrm{S} 2-1=0 . \backslash \mathrm{S} 2 \mathrm{~A}=0 . \backslash \mathrm{RMSD}=5.775 e-09 \backslash \mathrm{RMSF}=2.295 e-06 \backslash \mathrm{Dipole}=-2.36765$ $48,0.6234323,0.0526709 \backslash$ Quadrupole $=-5.400549,2.5535188,2.8470302,2.5616$ $984,-0.0026263,-0.3245259 \backslash \mathrm{PG}=\mathrm{C} 01 \quad[\mathrm{X}(\mathrm{C} 5 \mathrm{H} 8 \mathrm{O} 2)] \backslash \backslash \mathrm{Q}$

\begin{tabular}{|c|c|c|c|c|}
\hline Maximum & Force & 0.000001 & 0.000015 & YES \\
\hline RMS & Force & 0.000000 & 0.000010 & YES \\
\hline Maximum & Displacement & 0.000036 & 0.000060 & YES \\
\hline RMS & Displacement & 0.000006 & 0.000040 & YES \\
\hline
\end{tabular}

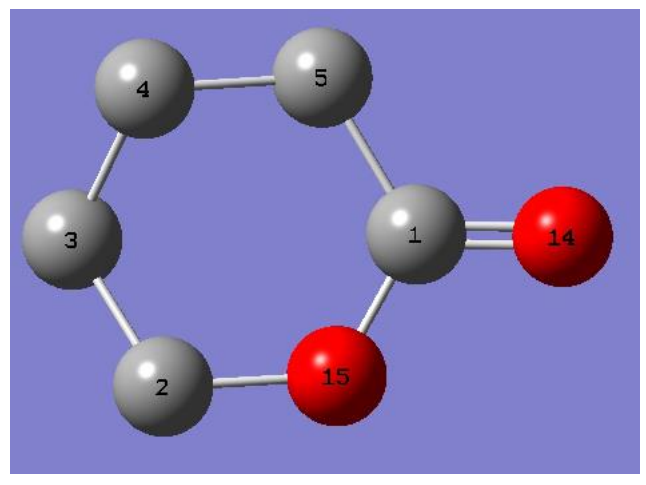




\section{d-Valerolactone radical ion(gas)}

SCF Done: E (UAPFD) =

Zero-point correction=

Thermal correction to Energy=

Thermal correction to Enthalpy=

Thermal correction to Gibbs Free Energy=

Sum of electronic and zero-point Energies=

Sum of electronic and thermal Energies=

Sum of electronic and thermal Enthalpies=

Sum of electronic and thermal Free Energies=
$-345.602770672$

0.124559 (Hartree/Particle)

0.130867

0.131812

0.093720

$-345.478212$

$-345.471903$

$-345.470959$

$-345.509051$

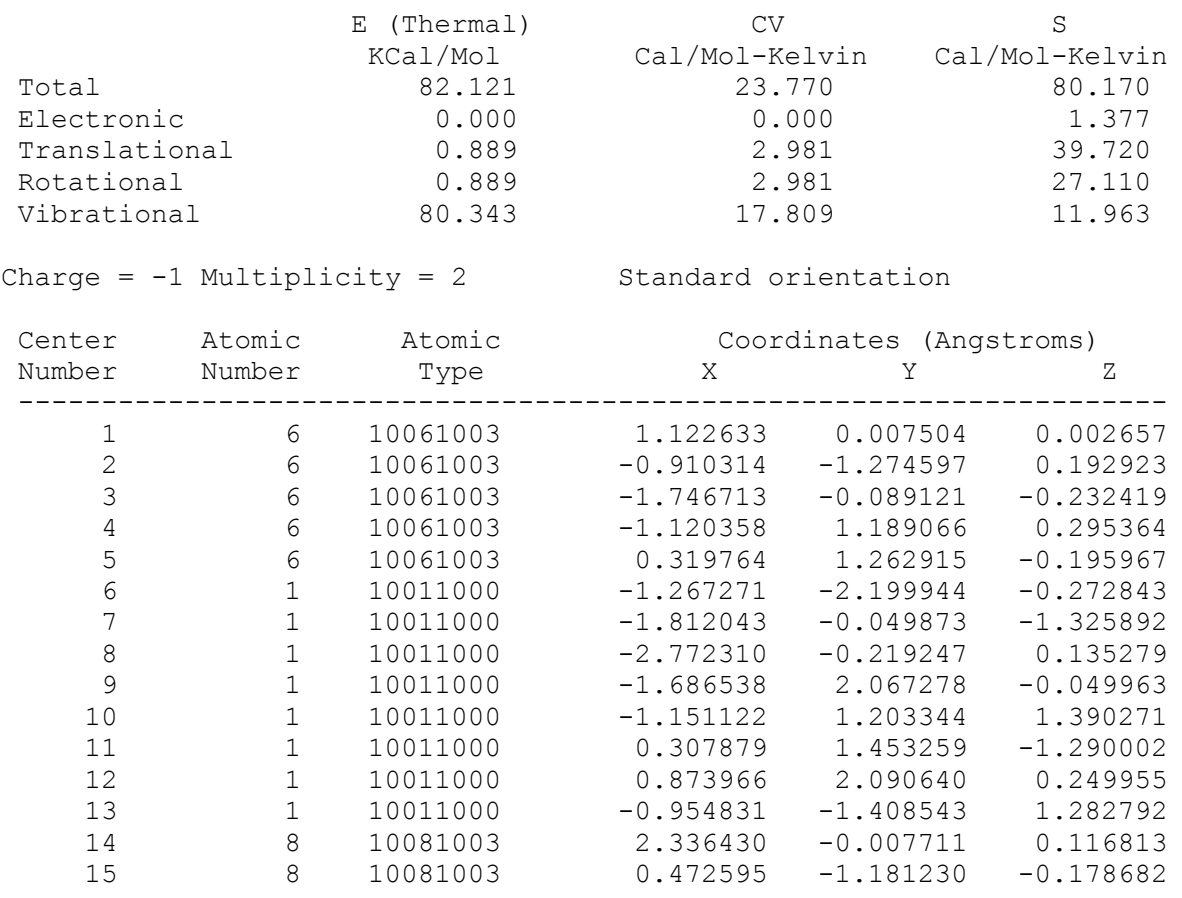

Summary of Natural Population Analysis:

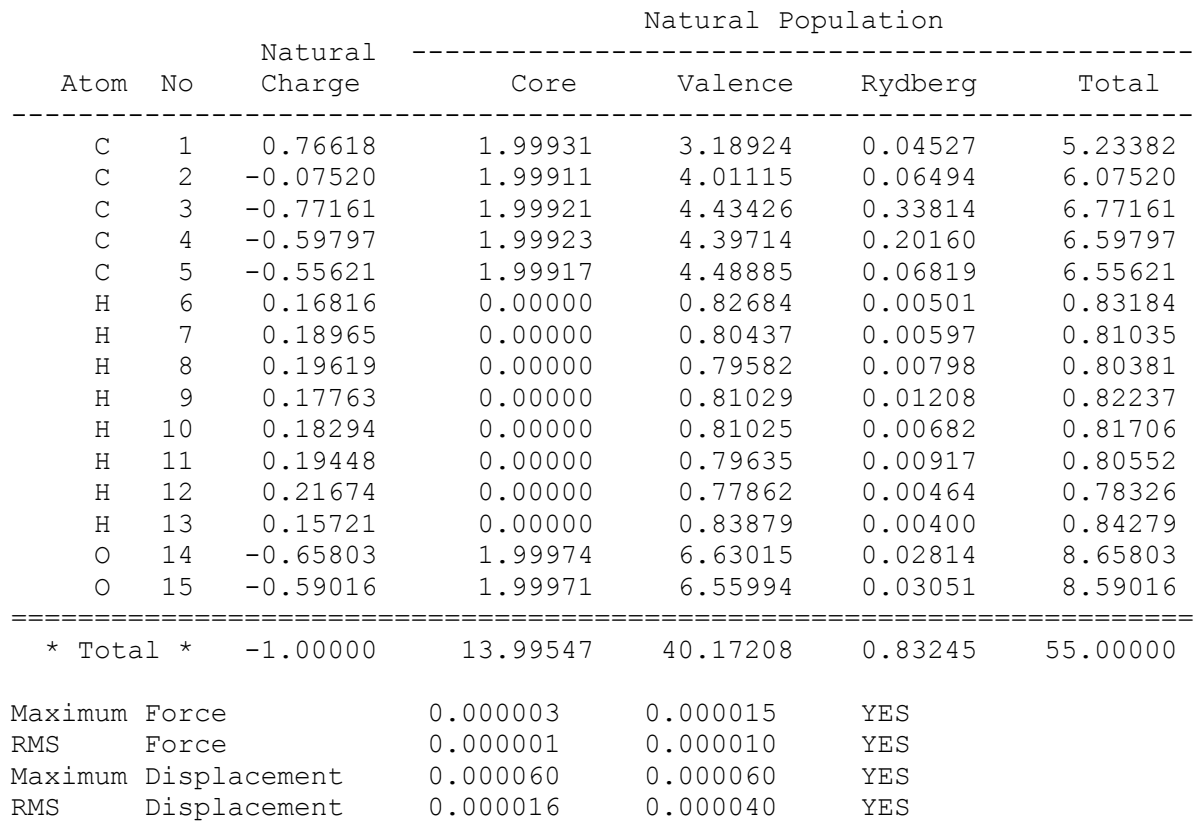


$1 \backslash 1 \backslash$ GINC-CORONA 42 $\backslash$ FOpt \UAPFD \6-311+G $(2 \mathrm{~d}, \mathrm{p}) \backslash \mathrm{C} 5 \mathrm{H} 802(1-, 2) \backslash$ WRA $0 \backslash 26-$ Apr -20 $16 \backslash 0 \backslash \backslash \#$ opt=(calcfc, tight) freq uapfd/6-311+g $(2 d, p)$ pop=npa geom=conne ctivity int=(ultrafine, $\operatorname{acc} 2 e=12) \backslash \backslash 1)$ Build dVL chair - clean - $>$ fname= dVL_MM0426.gjf 2)mod dVL_MM0426 to radical anion -- clean --> opt=(cal cfc,tight) uff int=ultrafine fname $=$ dVL radan MM0426.gjf 3) dVL radan MM $0426 . \log -->$ (M5) \# opt=(calcfC,tight) freq uapfd $/ 6-311+g(2 d, p)$ pop $=n$ pa geom=connectivity int=(ultrafine, acc2e=12) fname=dVL radan0426.gjf $\backslash$ $\backslash-1,2 \backslash C, 1.1124944114,-0.0274919726,0.0669004312 \backslash C,-0.97 \overline{3} 1301579,-1.229$ $3413265,0.2032260839 \backslash \mathrm{C},-1.7501385286,-0.0156288378,-0.2539291533 \backslash \mathrm{C},-1$. $0910352446,1.2412131038,0.2851150268 \backslash \mathrm{C}, 0.3648982758,1.2563831038,-0.16$ $30360118 \backslash \mathrm{H},-1.3514100774,-2.1435144966,-0.2677467014 \backslash \mathrm{H},-1.7812109651,0$ $.018540553,-1.349081635 \backslash \mathrm{H},-2.7905156635,-0.1037842494,0.0834551575 \backslash \mathrm{H},-$ $1.61237891,2.1380846475,-0.0821568891 \backslash \mathrm{H},-1.1539230864,1.2642245521,1.3$ $784975188 \backslash \mathrm{H}, 0.3930478001,1.4394799315,-1.258009701 \backslash \mathrm{H}, 0.9370189147,2.06$ $53067863,0.2946043106 \backslash \mathrm{H},-1.0553162667,-1.35394899,1.292012091 \backslash 0,2.3208$ $516042,-0.0884551619,0.2176477907 \backslash 0,0.4228338943,-1.1916466432,-0.1270$ 903189 \Version=EM64L-G09RevD.01 \State $=2-A \backslash H F=-345.6027707 \backslash S 2=0.750713$ $\backslash \mathrm{S} 2-1=0 . \backslash \mathrm{S} 2 \mathrm{~A}=0.75 \backslash \mathrm{RMSD}=5.414 \mathrm{e}-09 \backslash \mathrm{RMSF}=1.440 \mathrm{e}-06 \backslash \mathrm{Dipole}=0.6996487,-0.52$ $80011,0.4661551 \backslash$ Quadrupole $=-11.7973469,4.3925368,7.4048102,4.9565866,-$ $2.1193458,1.7688768 \backslash \mathrm{PG}=\mathrm{CO} 1 \quad[\mathrm{X}(\mathrm{C} 5 \mathrm{H} 8 \mathrm{O} 2)] \backslash \backslash @$

$\begin{array}{lllll}\text { Maximum } & \text { Force } & 0.000003 & 0.000015 & \text { YES } \\ \text { RMS } & \text { Force } & 0.000001 & 0.000010 & \text { YES } \\ \text { Maximum } & \text { Displacement } & 0.000055 & 0.000060 & \text { YES } \\ \text { RMS } & \text { Displacement } & 0.000016 & 0.000040 & \text { YES }\end{array}$

$\begin{array}{llll}\text { RMS Displacement } & 0.000016 & 0.000040 & \text { YES }\end{array}$

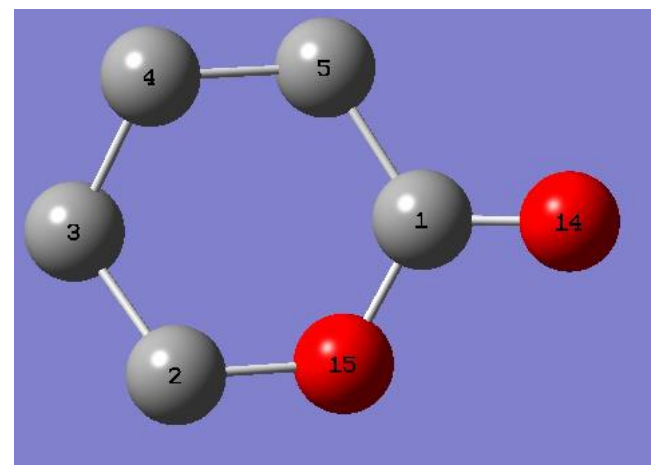




\section{d-Valerolactone radical ion (iefpcm)}

SCF Done: $\mathrm{E}(\mathrm{UAPFD})=$

Zero-point correction=

Thermal correction to Energy=

Thermal correction to Enthalpy=

Thermal correction to Gibbs Free Energy=

Sum of electronic and zero-point Energies=

Sum of electronic and thermal Energies=

Sum of electronic and thermal Enthalpies=

Sum of electronic and thermal Free Energies=
$-345.683074957$

0.124234 (Hartree/Particle)

0.130368

0.131313

0.093781

$-345.558841$

$-345.552707$

$-345.551762$

$-345.589294$

$\begin{array}{lccc} & \text { E (Thermal) } & \text { CV } & \text { S } \\ \text { KCal/Mol } & \text { Cal/Mol-Kelvin } & \text { Cal/Mol-Kelvin } \\ \text { Total } & 81.807 & 23.721 & 78.993 \\ \text { Electronic } & 0.000 & 0.000 & 1.377 \\ \text { Translational } & 0.889 & 2.981 & 39.720 \\ \text { Rotational } & 0.889 & 2.981 & 27.150 \\ \text { Vibrational } & 80.030 & 17.760 & 10.746\end{array}$

Charge $=-1$ Multiplicity $=2 \quad$ Standard orientation

\begin{tabular}{|c|c|c|c|c|c|}
\hline \multirow{2}{*}{$\begin{array}{l}\text { Center } \\
\text { Number }\end{array}$} & \multirow{2}{*}{$\begin{array}{l}\text { Atomic } \\
\text { Number }\end{array}$} & \multirow{2}{*}{$\begin{array}{c}\text { Atomic } \\
\text { Type }\end{array}$} & \multicolumn{3}{|c|}{ Coordinates (Angstroms) } \\
\hline & & & $\mathrm{X}$ & $\mathrm{Y}$ & Z \\
\hline 1 & 6 & 10061003 & 1.119036 & 0.014661 & 0.210799 \\
\hline 2 & 6 & 10061003 & -0.906059 & -1.270847 & 0.217495 \\
\hline 3 & 6 & 10061003 & -1.758870 & -0.094555 & -0.240420 \\
\hline 4 & 6 & 10061003 & -1.129069 & 1.207332 & 0.240600 \\
\hline 5 & 6 & 10061003 & 0.339323 & 1.260785 & -0.177668 \\
\hline 6 & 1 & 10011000 & -1.303177 & -2.217416 & -0.164512 \\
\hline 7 & 1 & 10011000 & -1.815362 & -0.100171 & -1.336314 \\
\hline 8 & 1 & 10011000 & -2.781121 & -0.209688 & 0.139785 \\
\hline 9 & 1 & 10011000 & -1.677857 & 2.074287 & -0.146800 \\
\hline 10 & 1 & 10011000 & -1.193080 & 1.251952 & 1.335506 \\
\hline 11 & 1 & 10011000 & 0.396495 & 1.380823 & -1.280632 \\
\hline 12 & 1 & 10011000 & 0.840049 & 2.131191 & 0.256734 \\
\hline 13 & 1 & 10011000 & -0.918875 & -1.318055 & 1.319626 \\
\hline 14 & 8 & 10081003 & 2.381151 & -0.030706 & 0.033089 \\
\hline 15 & 8 & 10081003 & 0.427195 & -1.181441 & -0.236617 \\
\hline
\end{tabular}

Summary of Natural Population Analysis:

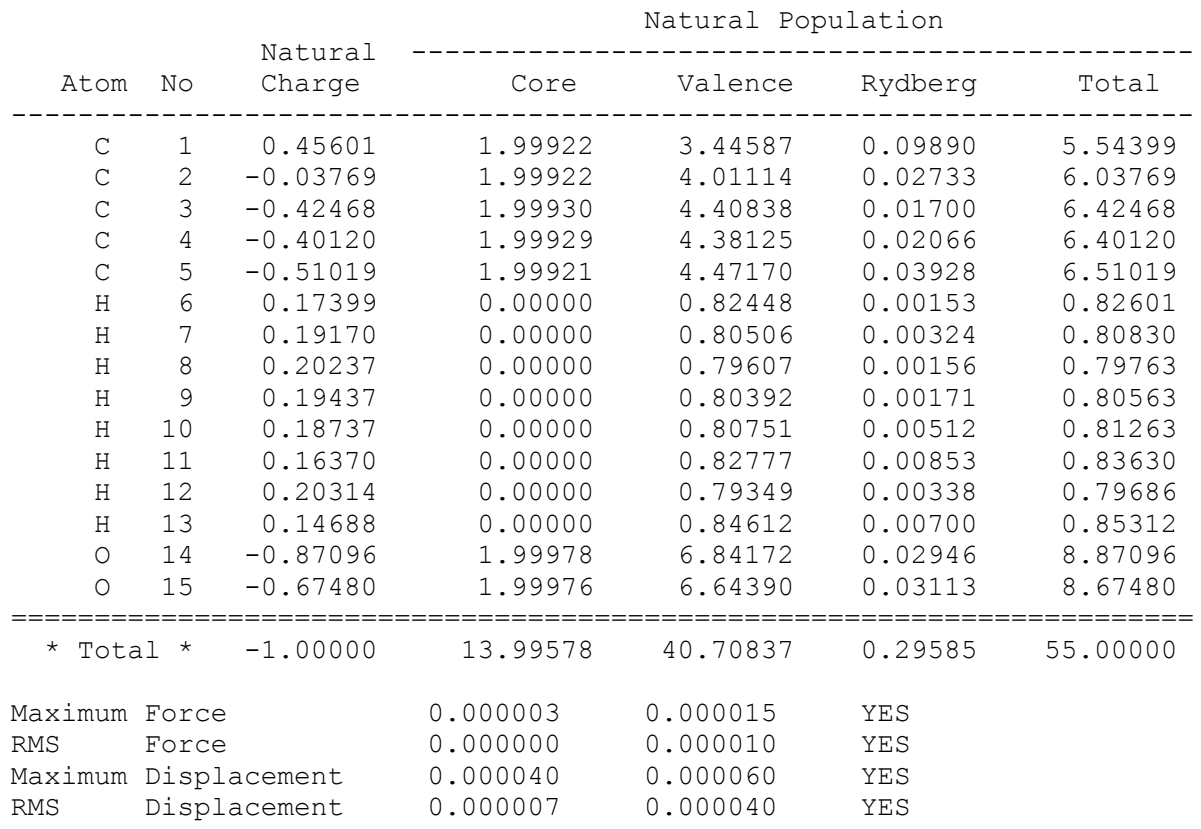


$1 \backslash 1 \backslash$ GINC-CORONA31 \FOpt \UAPFD \6-311+G $(2 \mathrm{~d}, \mathrm{p}) \backslash \mathrm{C} 5 \mathrm{H} 802(1-, 2) \backslash$ WRA $0 \backslash 27-A p r-20$ $16 \backslash 0 \backslash \backslash \#$ opt=(calcfc, tight) freq uapfd/6-311+g(2d,p) scrf=(iefpcm, solve $\mathrm{nt}=$ thf) pop=npa geom=connectivity int=(ultrafine, acc2e=12) \\1) Build dV L chair - clean --> fname=dVL_MM0426.gjf 2) mod dVL_MM0426 to radical a nion -- clean --> opt=(calcfc,tight) uff int=ultrafine fname=dVL radan _MM0426.gjf 3) dVL_radan_MM0426.gjf-->opt=(calcfC,tight) uff scrf=(iefp $\mathrm{cm}$, solvent=thf) int=ultrafine fname $=d V L-r a d a n$ MMpcm0427.gjf 4)dVL-rada n_MMpcm0427.log --> (M6) opt=(calcfc,tight) freq uapfd/6-311+g(2d,p) s $\mathrm{crf}=($ iefpcm, solvent=thf) $\mathrm{pop}=\mathrm{npa}$ geom=connectivity int=(ultrafine, acc2 $\mathrm{e}=12$ ) fname $=d V L-r a d a n \_p c m 0427 . g j f \backslash \backslash-1,2 \backslash C, 1.1059081357,-0.0217998757,0$ $.2478358552 \backslash \mathrm{C},-0.9613 \overline{4} 45244,-1.2367669294,0.1853112896 \backslash \mathrm{C},-1.7645339654$ $,-0.0215520284,-0.2605305392 \backslash \mathrm{C},-1.1006140004,1.2464068279,0.2633305343$ $\backslash \mathrm{C}, 0.3767503443,1.2590630244,-0.1255310979 \backslash \mathrm{H},-1.3826760178,-2.16000878$ $,-0.2266722657 \backslash \mathrm{H},-1.7994426388,0.0002157772,-1.3571223173 \backslash \mathrm{H},-2.7974383$ $898,-0.1102820222,0.0973009882 \backslash \mathrm{H},-1.611901254,2.1404677254,-0.11363009$ $6 \backslash \mathrm{H},-1.1847724933,1.2677734523,1.3575736145 \backslash \mathrm{H}, 0.4598189289,1.402635365$ $9,-1.2240278287 \backslash \mathrm{H}, 0.8979893072,2.1014298405,0.3389751058 \backslash \mathrm{H},-0.99760481$ $69,-1.3090770457,1.2855571608 \backslash 0,2.3690339137,-0.1063747783,0.093136616$ $4 \backslash 0,0.382916471,-1.1827135541,-0.2410910201 \backslash \backslash$ Version=EM64L-G0 9RevD.01\ State $=2-\mathrm{A} \backslash \mathrm{HF}=-345.683075 \backslash \mathrm{S} 2=0.753719 \backslash \mathrm{S} 2-1=0 . \backslash \mathrm{S} 2 \mathrm{~A}=0.750008 \backslash \mathrm{RMSD}=3.507 \mathrm{e}-$ $09 \backslash \mathrm{RMSF}=1.218 \mathrm{e}-05 \backslash \mathrm{Dipole}=-3.756225,0.7019504,-0.1118566 \backslash$ Quadrupole $=-8$. $9722781,4.4248415,4.5474366,2.4546129,-0.4557977,-0.2666433 \backslash P G=C 01 \quad[X($ $\mathrm{C} 5 \mathrm{H} 8 \mathrm{O} 2) \mathrm{l} \backslash \backslash \mathrm{e}$

\begin{tabular}{|c|c|c|c|c|}
\hline Maximum & Force & 0.000003 & 0.000015 & YES \\
\hline RMS & Force & 0.000000 & 0.000010 & YES \\
\hline Maximum & Displacement & 0.000036 & 0.000060 & YES \\
\hline RMS & Displacement & 0.000006 & 0.000040 & YES \\
\hline
\end{tabular}

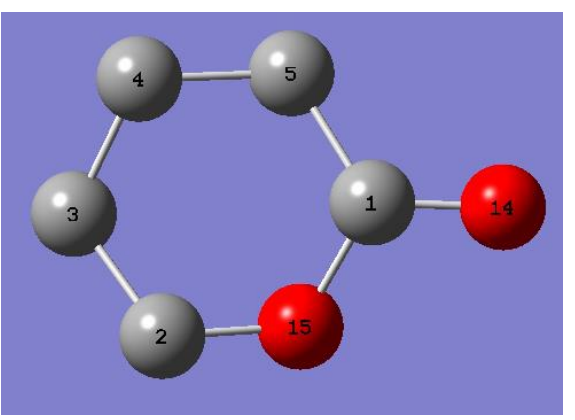

